\title{
Vasohibin 2 Promotes Lymphangiogenesis of Lung Squamous Cell Carcinoma Through Snail- dependent VEGF-D Signaling Pathway
}

\section{Pengpeng Liu}

Tianjin Medical University Cancer Institute and Hospital: Tianjin Tumor Hospital

\section{Rui Zhang}

Tianjin Medical University Cancer Institute and Hospital: Tianjin Tumor Hospital

Lei Han

Tianjin Medical University Cancer Institute and Hospital: Tianjin Tumor Hospital

Xiao Zhang

Tianjin Medical University Cancer Institute and Hospital: Tianjin Tumor Hospital

\section{Yingnan Ye}

Tianjin Medical University Cancer Institute and Hospital: Tianjin Tumor Hospital

\section{Wenwen Yu}

Tianjin Medical University Cancer Institute and Hospital: Tianjin Tumor Hospital

\section{Xiubao Ren}

Tianjin Medical University Cancer Institute and Hospital: Tianjin Tumor Hospital

\section{Weijia Zhang}

Icahn School of Medicine at Mount Sinai

Jinpu Yu ( $\nabla$ jyu@tmu.edu.cn )

Tianjin Medical University Cancer Institute and Hospital: Tianjin Tumor Hospital

\section{Research}

Keywords: Vasohibin 2, Lymphangiogenesis, LUSC, Snail, VEGF-D

Posted Date: December 15th, 2020

DOI: https://doi.org/10.21203/rs.3.rs-125315/v1

License: (c) (i) This work is licensed under a Creative Commons Attribution 4.0 International License. Read Full License 


\section{Abstract}

Backgroumd: Tumor metastasis is a process in which tumor cells enter the lymphatic vessels and blood vessels and then spread to the secondary site where they form secondary tumors. In vascular biology, angiogenesis and anti-angiogenesis therapy have been extensively studied, however, the molecular mechanisms involved in lymphangiogenesis and lymphatic metastasis remain unclear.

Methods: We analyzed mRNA expression profiles of 937 primary lung squamous cell carcinoma (LUSC) samples from The Cancer Genome Atlas (TCGA) and Gene Expression Omnibus (GEO) databases to screen the most differentially expressed genes related to the poor prognosis of LUSC patients and validated in an independent Chinese LUSC cohort. We focused on Vasohibin 2 (VASH2) and investigated its biological functions in LUSC proliferation, apoptosis, migration, invasion, as well as lymphangiogenesis by forced over-expressing VASH2 in LUSC cell line H520 in vitro. We also investigated the anti-tumor efficacy of VASH2 target treatment in LUSC xenograft-bearing mice models.

Results: We identified 12 genes closely related to poor prognosis of LUSC patients, among which VASH2 was validated in an independent Chinese LUSC cohort and displayed high potential of lymphatic metastasis. VASH2 promoted the proliferation and invasion of LUSC cells both in vitro and vivo. Forced over-expression of VASH2 in LUSC cells promoted the amplification and tube-formation of human umbilical vein endothelial cells (HUVECs) and human lymphatic endothelial cells (HLECs) cells via upregulating vascular endothelial growth factor-D (VEGF-D) production which could be reversed by Snail inhibition. Furthermore, blocking VASH2/VEGF-D signaling using specific antibodies dramatically inhibited tumor growth in mice by interfering proliferation of cancer cells and lymphangiogenesis in tumor tissues.

Conclusion: In conclusion, VASH2 facilitated lymphangiogenesis and tumor growth in a Snail-dependent manner which might serve as a novel biomarker for early diagnosis and prognosis prediction, as well as a potential therapeutic target in LUSC.

Statement of conflict of interest: The authors declare no potential conflicts of interest.

\section{Backgroumd}

Lung cancer is one of the most common human malignancies with high incidence and mortality worldwide [1]. The 5-year survival rate of lung cancer is only about $18.1 \%[2,3]$, mainly due to its aggressive invasion and rapid progression [1]. Non-small cell lung cancer (NSCLC) accounts for the majority (>85\%) of all lung cancers, among which lung squamous cell carcinoma (LUSC) accounts for about $30 \%$ cases but has little benefit from targeted therapies compared to lung adenocarcinoma (LUAD) for less druggable target biomarkers [4]. Thus, there is an urgent need to uncover the molecular mechanisms involved in aggressive invasion and metastasis of LUSC in order to develop novel therapeutic strategies with high efficiency for LUSC. 
Invasion and metastasis are a complex dynamic process involved multiple factors, which mainly occurs through blood vessels and lymphatic vessels. In LUSC, some studies have illuminated lymphangiogenesis could enhance the affinity of tumor cells to lymphatic vessels, further promoting invasion and metastasis through lymphatic vessels [5]. However, the mechanisms of lymphangiogenesis in LUSC development and progression are still unclear. Considering LUSC metastasis initiated from invading into lymph nodes through lymphatic vessels [6,7], specific interdiction of lymphangiogenesis would provide more efficient inhibition on LUSC invasion compared to routine anti-angiogenesis treatment. Therefore, to discriminate the key biomarkers regulating the process of lymphangiogenesis in LUSC will throw a light on developing novel anti-cancer therapeutic strategies for LUSC [8-10].

In previous study, we analyzed mRNA expression profiles of 937 primary LUSC samples from GEO and TCGA databases and filtered out VASH2 as one of the clinical beneficial predictable biomarkers associated with LUSC poor prognosis. The gene for human VASH2 is located on chromosome 1q32.3. The full-length human VASH2 is composed of 355 amino acid residues [11]. It was initially reported to be expressed in endothelial cells. VASH2 is mainly expressed in monocytes (MNCs), which are mobilized from bone marrow and infiltrated into the front of germination [12].

VASH2 has been demonstrated to be an angiogenic factor in a variety of tumors, including liver cancer, gastric cancer, breast cancer, and ovarian cancer [13-15], and plays an important role in tumor development [16]. However, none study on the expression and function of VASH2 in lung cancer, especially in LUSC has ever been reported. It has been reported that exogenous VASH2 significantly promoted tumor growth, increased microvessel density and hemoglobin concentration [17], while tumor angiogenesis was significantly inhibited in VASH2 knockout mice [18]. Furthermore, VASH2 was reported to trigger epithelial-mesenchymal transition (EMT), up-regulate chemotherapeutic resistance, and increase the proportion of cancer stem like cells $[19,20]$ which can generate cellular traits associated with more aggressive malignancy with higher motility and invasiveness by imparting the trait of self-renewal to cancer cells. However, whether VASH2 promotes invasion and metastasis of lung cancer is related to angiogenesis or EMT has not been disclosed.

In this study, we explored the clinical significance of VASH2 in LUSC, and potential molecular mechanisms involved in VASH2 promoting the development and progression of LUSC. We demonstrated that high level of VASH2 was significantly associated with poor prognosis and promoted lymphatic metastasis in patients with LUSC. It was confirmed from cell experiments and animal models that overexpression of VASH2 promoted angiogenesis and lymphangiogenesis, and accelerated the invasion and metastasis of tumor cells by stimulating EMT. The expression of VASH2 can significantly increase the production of Snail and VEGF-D in LUSC cells. And blocking VASH2/VEGF-D signaling pathway could significantly inhibited the growth of tumor and the formation of lymphatics. In summary, we not only revealed VASH2 as a marker of LUSC, but also proposed new molecular mechanisms of lymphatic metastasis, which provided potential molecular diagnosis and targeted treatment strategies for patients with LUSC. 


\section{Methods}

\section{Reagents and antibodies}

RPMI1640 (C11875500BT), DMEM/F12 (11330032), fetal bovine serum (10099141), Trypsin (25200072) and antibiotic-Antimycotic (15240062) were purchased from Gibco BRL (Grand Island, NY, USA). FITC BrdU Flow kit (559619), Annexin-V-FITC Apoptosis Detection kit (556547) and Matrigel (356230) were purchased from BD Biosciences (San Jose, CA, USA). Trizol (15596026) were purchased from Invitrogen (Grand Island, NY, USA). ELISA kit to detect secretion of VEGF-D (ELH-VEGFD-001) was purchased from RayBiotech (Norcross, GA, USA). ELISA kit to detect secretion of VASH2 (ab155288) was purchased from Abcam (Cambridge, UK). DMSO (D2650) was purchased from Sigma (St. Louis, MO, USA). Oligo-(dT)15 primer, $10 \mathrm{mM}$ dNTP, RNase inhibitor, SYBR Premix Ex Taq ${ }^{\text {TM }}$ kit (RR820A) were purchased from Takara (Tokyo, Japan). In flow cytometry assay, all fluoresce-labeled antibodies were purchased from Biolegend (San Diego, CA, USA), including PE anti-human Podoplanin Lymphoendothelial cell surface markers (LEC), and PE-IgG1. In immunohistochemistry, all antibodies were purchased from Bioss (Beijing, China) and biotinylated secondary goat anti-mouse or rabbit IgG antibody was purchased fromSanta Cruz (Dallas, TX, USA).

\section{Mice}

BALB/c female mice between 6-8-week-old were purchased from Beijing SPF Biotechnology Co., Ltd. Mice were maintained at SPF animal laboratory of Tianjin Medical University Cancer Hospital and Institute. To establish the lung adenocarcinoma mouse models, the mouse lung adenocarcinoma cells $\mathrm{H} 52 \mathrm{O}^{\mathrm{CTRL}}$ and $\mathrm{H} 520^{\mathrm{OV}-\mathrm{VASH} 2}$ ( $1 \times 10^{6}$ cells in $\left.100 \mathrm{ul} \mathrm{PBS}\right)$ was injected into the mammary fat pads of BALB/c mice to induce tumor formation [1]. All procedures that involve mice were approved by the Ethics Committee for Animal Experiments at the Tianjin Medical University Cancer Hospital and Institute, and were performed in accordance with the Guide for the Care and Use of Laboratory Animals.

\section{Cell Lines}

Human lung adenocarcinoma cell line H520 was obtained from Chinese Academy of Medical Sciences tumor cell libraries.

\section{Cell line culture}

The human LUSC cell line H520 was cultured in RPMI 1640 complete medium containing $10 \%$ fetal bovine serum in at $37^{\circ} \mathrm{C}, 5 \% \mathrm{CO}$. By transfecting wild-type $\mathrm{H} 520$ cells (H520 CTRL) with VASH2-specific short hairpin RNA (shRNA) lentiviral vectors, $\mathrm{H} 520^{\mathrm{OV}-\mathrm{VASH} 2}$ cells were established as indicated in our previous work. Stably transfected cells were cloned by limiting dilution and validated using RT-PCR and Western blot as our previous work described.

\section{Clinical samples}


This study recruited 64 cases of LUSC patients treated with partial lung resection surgery at the Department of Hepatobiliary Oncology of the Tianjin Medical University Cancer Institute and Hospital from November 2004 to November 2006. These patients included 49 males and 19 females with a median age of 62.7 years. No prior treatments, including chemotherapy or radiotherapy, were conducted before lung resection surgery was performed. Postoperative follow-up time was 40-65 months. This project was approved by the Ethics Committee of Tianjin Medical University. All experiments were performed in accordance with the principles of the Declaration of Helsinki. Written consents were obtained from the patients. Tissue samples were flash frozen and stored at $80^{\circ} \mathrm{C}$.

\section{Data Processing for Public Datasets}

Public datasets were downloaded from the GEO and TCGA database. 10 studies of LUSC expression profiling with a minimum of 24 LUSC patients were collected from GEO database for the meta-analysis study, including 41 dataset from GSE50081, 78 dataset from GSE41271, 55 dataset from GSE30219, 24 dataset from GSE19188, 72 dataset from GSE8894, 43 dataset from GSE42127, 53 dataset from GSE3141, 52 dataset from GSE14814, 66 dataset from GSE37745, 38 dataset from Tianjin database and 415 dataset from TCGA. Detailed information for these datasets is listed in Figure S1.

The workflow for the meta-analysis of the discovery set of 1 Tianjin and 10 public LUSC datasets was depicted in Figure S1. Meta genes associated with short survival (<5 years: 5 years was the median survival of 11 cohorts) and long survival ( $>5$ years) were identified by a meta p-value of less than 0.05 by combining $p$ values from 11 datasets and individual $p$ value $<0.05$ in at least 4 out of 11 datasets from the 11 datasets, . The meta genes were subjected to Gene Ontology (GO) and canonical pathway enrichment with multiple pathways databases (KEGG, Reactome, PID, Wiki Pathways, Biocarta and Panther).

\section{Immunohistochemistry}

All samples were heated for $0.5 \mathrm{~h}$ at $56^{\circ} \mathrm{C}$, deparaffinized in xylene and rehydrated through graded alcohol. Antigens were retrieved by heating in citrate buffer ( $\mathrm{pH}$ 6.0) for $20 \mathrm{~min}$. Endogenous peroxidase activity was quenched in a bath of methanol and hydrogen peroxide for $30 \mathrm{~min}$. The samples were then incubated overnight at $4^{\circ} \mathrm{C}$ with mouse antibody, and a biotinylated secondary goat anti-mouse or rabbit IgG antibody labeled with streptavidin-horseradish peroxidase (HRP) using a DAB staining kit according to the manufacturer's instructions. Five representative high-power fields (400x magnification) were selected for each tissue section for histological evaluation. For each protein, two parameters, namely, positive rate (PR) and staining intensity (SI), were used to describe the expression on the basis of the extensity and intensity of the positively stained cells in the samples. PR denotes the percentage of positively stained cells in cancer tissues: $\leq 15 \%$, negative (scored as 0 ); $16 \%-50 \%$, positive at low frequency (scored as 1); 51\%-80\%, positive at medium frequency (scored as 2); and $\geq 80 \%$, positive at high frequency (scored as 3 ). SI refers to the ranked staining intensity of positively stained cells in LUSC samples. This value ranged from 0 to 3, which corresponded to negative, weakly positive, moderately positive, and strongly positive. The sum represented the final scores of each protein marker in the 
samples because protein expression was comprehensively evaluated on the basis of both parameters. A final score of $<4$ was defined as low/negative expression and a final score of $\geq 4$ was defined as high expression.

\section{Lentivirus construction}

The vector冈pHBLV-CMV-MCS-3FLAG-EF1-ZsGreen-T2A-PURO $₫$ was digested at $37^{\circ} \mathrm{C}$ and the gel was recovered. Then the fragments were recovered by PCR. The target fragment was connected with the vector and the reaction system(20ul). 100 ulcompetent cell suspension was taken from $-70^{\circ} \mathrm{C}$ refrigerator and thawed at room temperature. Add plasmid DNA solution, shake gently, place on ice for 30 minutes, heat shock in $42^{\circ} \mathrm{C}$ water bath for 90 seconds and quickly place on ice to cool for 3-5 minutes after heat shock. $1 \mathrm{ml}$ LB liquid medium (without antibiotics) was added into the tube, and then it was stirred at $37^{\circ} \mathrm{C}$ for 1 hour in a 220 RPM shaker. The bacteria were restored to normal growth state and expressed antibiotic resistance gene encoded by plasmid. After shaking the above bacterial solution, centrifugation was performed to remove $900 \mathrm{ul}$ supernatant. After the remaining culture medium was sucked and mixed, 100ul was applied to the screening plate containing antibiotics. The plate was placed upside down for half an hour. After the bacterial solution was completely absorbed by the medium, the plate was inverted and cultured at $37^{\circ} \mathrm{C}$ for $16-24$ hours. After the transformation, the plates were picked up and shaken for 14 hours at $230 \mathrm{RPM} / \mathrm{min}$ at $37^{\circ} \mathrm{C}$. The positive clones were sent to sequencing company for sequencing for construction of VAHS2 and Snail overexpression lentivirus. The synthesized VASH2 shRNA and Snail shRNA were inserted into plvx-U6-CMV-RFP-P2A-BSD and pHBLV-U6-MCS-CMVZsGreen-PGK-PURO lentiviral vectors respectively. The lentivirus was generated as described above.

\section{Tube formation}

$\mathrm{H} 520^{\mathrm{CTRL}}$ and $\mathrm{H} 520^{\mathrm{OV}-\mathrm{VASH} 2}$ cells were cultured as described above. When the cells reached $80 \%$ confluence, the culture medium was changed to DMEM without fetal bovine serum. After an additional $48 \mathrm{~h}$ culture, the supernatant was collected as $\mathrm{CM}$ and stored at $20^{\circ} \mathrm{C}$. After thawed at $4{ }^{\circ} \mathrm{C}$ overnight, the Matrigel was coated in 96-well plate then incubated at room temperature for at least $30 \mathrm{~min}$ to gel. HUVECs or HLECs were suspended at a density of $2 \times 10^{5}$ cells $/ \mathrm{ml}$ in the different CMs. The cell suspensions $(100 \mathrm{ml})$ were added to each Matrigel coated well. DMEM was substituted for CM for the negative control. DMEM and VASH2 factor were substituted for $\mathrm{CM}$ for the positive control. After $18 \mathrm{~h}$, the formed networks were photographed and analyzed to calculate the the formation rate of tubes was calculated according to the following equation: Formation rate $(\%)=$ Cell number Tube quantity $_{\text {Cell }}$ number ${ }_{\text {Total }} \times 100 \%$.

\section{RT-qPCR analysis for gene expression}

Total RNA was exacted using the Trizol kit according to the manufacturer's protocol. Complementary DNA was synthesized using equivalent amounts of total RNA $(1 \mu \mathrm{g})$ in a $20 \mu \mathrm{l}$ reverse transcriptase reaction mixture and detected using SYBR Premix Ex Taq ${ }^{\text {TM }}$ in 7500 Real-Time PCR System (Applied Biosystems, Foster City, CA, USA). The primers were synthesized from Santa Cruz (Dallas, TX, USA). The sequences of 
primers are shown in Table 2. All experiments were performed in triplicates and were calculated for $\triangle \mathrm{CT}$. $\triangle C T=C T_{\text {target gene }}-C T_{\text {reference gene. }}$ And relative expression quantity of mRNA is $2-\triangle C T$. Most of the PCR primer sequences used in this study were referenced from literatures (27-38). But for the primer sequences of 8 EMT-related genes (Zeb1, Zeb2, Twist1, Snail, Slug, $\beta$-catenin, N-cadherin and E-cadherin) and VEGF family genes (VEGF-A, VEGF-C and VEGF-D), they were designed by ourselves and validated in our previous work.

\section{Enzyme-linked immunosorbent assay (ELISA)}

Cells $\left(4 \times 10^{5}\right)$ were seeded into 6-well plates and the culture supernatants of $\mathrm{H}_{52} \mathrm{C}^{\mathrm{CTRL}}$ andH520 $\mathrm{OV}$-VASH2 cells were collected after $48 \mathrm{~h}$ to determine the VASH2 and VEGF-D levels using the VASH2/VEGF-D ELISA kit.

\section{Western blot analysis}

The cells were harvested and washed twice with PBS ( $\mathrm{pH} 7.4,0.15 \mathrm{M})$. Total protein was extracted by RIPA buffer (Beyotime, Shanghai, China). Approximately $30 \mu \mathrm{g}$ of total protein was subjected to SDSPAGE and transferred to PVDF membranes. The membranes were blocked with $5 \%$ skim milk in TBST and incubated with the primary antibody (1:1000) in TBST containing $5 \%$ BSA overnight at $4{ }^{\circ} \mathrm{C}$. The membranes were washed twice with TBST and incubated with HRP-conjugated secondary antibody (1:4000; Santa Cruz, Dallas, TX, USA) at room temperature for $2 \mathrm{~h}$. The membrane was exposed using an enhanced chemiluminescence reagent (Chemicon International, USA). The membranes were then reprobed with anti- $\beta$-actin antibody (Santa Cruz, Dallas, TX, USA) in 1:2000 dilutions to confirm equal protein loading. The antibodies to detect VASH2, VEGF-D, Snail, and actin were purchased from Cell Signaling Technology (MA, USA).

\section{Cell counting kit-8 (CCK-8) assay}

Cells $\left(8 \times 10^{3}\right)$ were seeded into 96 -well plates. CCK-8 solution $(10 \mu \mathrm{L}$; Dojindo Molecular Technologies, Inc., Rockville, MD, USA) was added to each well for different time periods $(2,24,72$, and $96 \mathrm{~h})$ and incubated for $2 \mathrm{~h}$ at $37^{\circ} \mathrm{C}$. The cell viability was represented by the absorbance measured at $450 \mathrm{~nm}$.

\section{Cell Apoptosis Analysis}

The apoptotic in $\mathrm{H} 520^{\mathrm{CTRL}}$ andH520 ${ }^{\mathrm{OV}-\mathrm{VASH} 2}$ cells was measured using Annexin-V-FITC. The Annexin-VFITC Apoptosis Detection kit was used to measure apoptosis. After 12 hours starvation treatment, cells were washed with PBS and resuspended in $1 \times$ binding buffer at a concentration of $1 \times 10^{6} \mathrm{cells} / \mathrm{ml}$. Subsequently, $5 \mu \mathrm{l}$ of Annexin-V and $10 \mu \mathrm{l}$ of PI were added to $100 \mu \mathrm{l}$ of the cell suspension, and the mixture was incubated for $15 \mathrm{~min}$ in the dark. The analyses were performed using a flow cytometry. Because the growth factors were solved in DMSO for cell culture experiment, DMSO was added into medium and diluted at the final concentration of $5 \mu \mathrm{g} / \mathrm{ml}$, serving as a negative control in order to eliminate the disturbance of solvent. The experiments were repeated at least 3 times. 


\section{Wound Healing Assay}

Cells were seeded into 6-well plates for $24 \mathrm{~h}$ to reach $80 \sim 90 \%$ confluence. The cell monolayer was wounded in a straight line with a $10 \mu \mathrm{l}$ pipette tip to create a "wound". Cell debris was removed by PBS and the wounded cells were cultured with fresh medium. Images were taken at $0,24 \mathrm{~h}$ and $48 \mathrm{~h}$ later to measure the distance of the wound. The cell migration rate $=$ (the distance of the wound at $0 \mathrm{~h}-$ the distance of the wound at $24 \mathrm{~h}$ or $48 \mathrm{~h}$ ) / the distance of the wound at $0 \mathrm{~h} \times 100 \%$. The experiments were repeated at least 3 times.

\section{Trans-well invasion assay}

The Matrigel and Trans-well were used to carry out lung cancer cell invasion assays to build invading chambers for separating high invasive and low invasive cells. Cells were seeded at a density of $1 \times 10^{5}$ cells in the Matrigel and 100 $\mu$ l serum-free RPMI-1640 to the 24-well plate Trans-well system with an 8- $\mu \mathrm{m}$ pore size polycarbonate filter membrane (Corning, NY, USA). The lower chambers contained $10 \%$ FBS containing medium. The cells were incubated for $48 \mathrm{~h}$. The cells on the lower surface of the membrane were fixed with methanol and stained with $1 \%$ toluidine blue. The stained membranes were photographed through the microscope and invading cells were counted. The experiments were repeated at least 3 times.

\section{In Vivo Tumorigenicity Study}

$\mathrm{H} 520^{\mathrm{CTRL}}$ andH520 $\mathrm{OV}$-VASH2 cells were collected and an equal number of $1 \times 10^{6}$ cells in $100 \mu$ l of PBS were mixed with Matrigel respectively. The mixed cells were injected subcutaneously into both flanks of BALB/c mouse. The tumor sizes were monitored every 3 days. Each group contains 5 mice. And the experiments were repeated at least 3 times. The tumor volume $(V)$ was calculated by the formula: $\mathrm{V}=3.14 \times \mathrm{L} \times \mathrm{W} \times \mathrm{H} / 6$ (L: length, $\mathrm{W}$ : width, $\mathrm{H}$ : height). The animal protocol used in this study was approved by the Ethics Committee for Animal Experiments of the Tianjin Medical University Cancer Hospital and Institute, and was performed in accordance with the Guide for the Care and Use of Laboratory Animals.

Once palpable tumors were formed (in 7-10 days) treatment cycles was initiated. The mice were anesthetized by intraperitoneal injection of $100 \mathrm{mg} / \mathrm{kg}$ anti-VEGFD and $10 \mathrm{mg} / \mathrm{kg}$ anti-VASH2. Treatment involved the mice being randomly divided into three groups, control, anti-VEGFD, and anti-VASH2-with each group containing 5 mice. The control group of mice were subjected to $1 \times$ PBS injections also for 5 consecutive days. After the completion of the treatment cycle the mice were sacrificed and the tumors were excised. Tumor volumes for the excised tumors were measured using a caliper and volumes were calculated as previously mentioned. Tumors were then fixed and processed for immunohistochemistry.

\section{Flow Cytometry}

The concentration of antibodies used for staining and the time of co-incubation were followed the manufacturer's instruction. PE anti-human Podoplanin antibody was used at the concentration of 12.5 $\mu \mathrm{g} / \mathrm{ml} .1 \times 10^{5}$ cells were resuspended in $100 \mu \mathrm{l}$ PBS, incubated for $30 \mathrm{~min}$ with different antibodies before 
washing and being fixed using $4 \%$ paraformaldehyde. Since all protein markers are membrane proteins, none cells were permeabilized. The samples were analyzed by BD FACS Aria flow cytometer (BD Biosciences).

\section{Data Analysis and Statistical Methods}

Data were analyzed using SPSS 17.0. Quantitative data were presented as mean \pm standard deviation. We performed Student's t-test when analyzing the statistical comparisons between two groups. Meanwhile, for more than 2 groups included in this study, we used one-factor ANOVA assay to evaluate the difference among all groups, followed by LSD method to compare the difference between each 2 groups. Statistical significance was set at $p<0.05$.

\section{Results}

\section{High expression of VASH2 correlated with worse prognosis and more lymphatic metastasis in LUSC patients}

We collected and analyzed the global gene expression profiling of 937 primary LUSC tissue samples from 11 database based on the disparity in clinical outcomes. We defined the patients with a survival time more than 40 months as good prognosis group, and those with a survival time less than 40 months as poor prognosis group. And we identified 12 highly differentially expressed genes which significantly correlated with patients' poor prognosis (Figure S1). We collected LUSC tissue samples from Tianjin Medical University Cancer Institute and Hospital (TJMUCH) to validate the correlation between the 12 identified genes and overall survival (OS) in 20 cases. The immunohistochemical (IHC) staining results and Kaplan-Meier survival analysis indicated that the patients with high expression of VASH2 and IL-33 showed a worse prognosis significantly (VASH2: $25.13 \pm 11.57$ vs. $52.42 \pm 30.65$ months, $p=0.023$; IL-33: $30.55 \pm 8.39$ vs. $50.45 \pm 21.18$ months, $p=0.032$; Figure $1 \mathrm{~A})$. Among them, Overexpression ofVASH2 was significantly correlated with the poor prognosis in 4 of 11 databases, indicating that VASH2 is a novel prognostic factor of LUSC whose high expression was significantly correlated with comparably shorter OS in different databases (Figure 1B). The finding of TCGA data implied that VASH2, previously reported to be associated with angiogenesis, might be a potential prognosis predictive biomarker for LUSC which was significantly up-regulated in LUSC cases suffering from shorter OS.

In order to validate the correlation between VASH2 and clinical outcome in LUSC, we collected another independent cohort composing of 64 LUSC samples from TJMUCH. The mRNA level of VASH2 in LUSC samples was detected using real-time quantitative PCR (qPCR) assay. We observed that low VASH2 expression was mainly found in patients survived more than 40 months, while level of VASH2 mRNA of good prognosis group is much lower than that of poor prognosis group $(10.68 \pm 1.13$ vs. $10.14 \pm 0.96$, $\mathrm{p}=0.01$ ॠigure $1 \mathrm{C}$ ). In addition, we compared the level of VASH2protein in the corresponding LUSC paraffin specimens using IHC analysis, and found that VASH2 protein was predominately higher in patients with short OS compared to that in patients with long OS $(63.82 \pm 5.18 \%$ vs. $20.88 \pm 3.74 \%, p=0.01$,

Figure 1D). Above results indicated that both the transcription and expression of VASH2 gene in LUSC are 
significantly correlated with poor survival outcomes consistently. Furthermore, we analyzed the association between VASH2 and other clinicopathology and found that high expression of VASH2 positively corresponds with more lymph node metastasis, lager tumor size, and worse clinical stage (Figure 1E, Table 1). These results indicated that VASH2 was a valuable biomarker to predict poor prognosis and lymphatic metastasis in LUSC.

\section{VASH2 accelerated the proliferation and invasion potential of LUSC cells, and promoted tube-formation capacity of HUVEC and HLEC cells}

Then, the supernatants from cultures of $\mathrm{H} 520^{\mathrm{CTRL}}$ and $\mathrm{H} 520^{\mathrm{OV}-\mathrm{VASH} 2}$ were collected. In order to investigate the underlying biological roles of VASH2 in carcinogenesis and lymphangiogenesis, VASH2 was up-regulated in LUSC cell line $\mathrm{NCl}-\mathrm{H} 520$ cells by transfecting recombinant lentivirus (Figure S2A). High level of VASH2 was confirmed in the supernatants of $\mathrm{H} 520^{\mathrm{OV}-\mathrm{VASH} 2}$ compared to that inH520 CTRL. The level of VASH2 protein in different supernatants was detected by ELISA. We found H520 ${ }^{\mathrm{OV}}$-VASH2 secreted more VASH2 than H520 CTRL $(602.75 \pm 73.84 \mathrm{pg} / \mathrm{ml}$ vs. $196.39 \pm 38.29 \mathrm{pg} / \mathrm{ml}, \mathrm{p}=0.001$, Figure $2 \mathrm{~A})$.

We first compared the proliferation and apoptosis of $\mathrm{H} 520^{\mathrm{OV}-\mathrm{VASH} 2}$ and found higher proliferation but lower apoptosis rates in $\mathrm{H} 520^{\mathrm{OV}-\mathrm{VASH} 2}$ compared to those in $\mathrm{H} 520^{\mathrm{CTRL}}$ (72h: $3.42 \pm 0.43$ vs. $2.19 \pm 0.36$, $p=0.044$, Figure $2 B) .(6.94 \pm 1.68 \%$ vs. $15.23 \pm 0.98 \%, p=0.013$, Figure $2 C)$. The migration and invasion potentials were compared. The wound closure rates (WCRs) were significantly higher in $\mathrm{H} 520 \mathrm{OV}$ VASH2 cells and more $\mathrm{H} 520^{\mathrm{OV}-\mathrm{VASH} 2}$ migrated across the Matrigel layer after $48 \mathrm{~h}(77.89 \pm 7.64 \% \mathrm{vs}$. $56.25 \pm 5.47 \%, p=0.016$, Figure 2D), and (228.67 \pm 16.77 vs. $174.33 \pm 12.50, p=0.011$, Figure $2 E)$.

Next, HUVECs and HLECs were co-cultured with the supernatants from either $\mathrm{H} 520^{\mathrm{OV}-\mathrm{VASH} 2}$ or $\mathrm{H} 520^{\mathrm{CTRL}}$ cells. The HUVECs and HLECs cultured in DMEM medium were used as negative controls. The numbers of forming tubes in each group were assessed. The results showed that when recombinant VASH2 was added to the supernatant, HUVECs and HLECs could form more tubes (HUVEC: $15.38 \pm 3.41 \%$ vs. $32.39 \pm 8 \% 47, p=0.032$; HLEC: $20.54 \pm 3.17 \%$ vs. $47.64 \pm 8.64 \%, p=0.007$, Figure $2 F-G)$. The typical tubule formation of HUVEC and HLEC cells was inhibited and reduced by adding specific VASH2 blocking antibody (HUVEC: $32.39 \pm 8.47 \%$ vs. $8.65 \pm 2.64 \%$, p=0.0098; HLEC: $47.64 \pm 8.64 \%$ vs. $12.19 \pm 3.62 \%$, $\mathrm{p}=0.0028$, Figure $2 \mathrm{~F}-\mathrm{G}$ ). The H520 $\mathrm{OV}-\mathrm{VASH} 2$ supernatant could stimulated both HUVEC and HLECs cells to generate comparable amount of tubes as recombinant VASH2 did in vitro, which was significantly higher than the H520 ${ }^{\mathrm{CTRL}}$ supernatant did (HUVEC: $39.64 \pm 7.31 \%$ vs. $21.39 \pm 4.35 \%, \mathrm{p}=0.021$; HLEC: $43.67 \pm 7.64 \%$ vs. $14.67 \pm 5.37 \%, p=0.0058$, Figure $2 \mathrm{H}-\mathrm{I})$. But after adding specific VASH2 blocking antibody, the typical tube formation of HUVEC cells was partly inhibited while the tube formation of HLECs was fully eliminated (HUVEC: $25.36 \pm 4.39 \%$ vs. $39.64 \pm 7.31 \%$, $p=0.043$; HLEC: $16.23 \pm 8.73 \%$ vs. $43.67 \pm 7.64 \%$, $\mathrm{P}=0.015$, Figure $2 \mathrm{H}-\mathrm{I})$. Above results implied that $\mathrm{VASH} 2$ not only effectively promoted proliferation and invasion potential of LUSC cells, but also significantly enhanced lymphangiogenesis in vitro.

\section{VASH2 facilitated lymphangiogenesis via up-regulating VEGF-D production in LUSC cells}


In order to define the molecular events regulating VASH2-related lymphangiogenesis, the transcription and expression of lymphangiogenesis-related VEGF family members inH520 ${ }^{\mathrm{OV}-\mathrm{VASH} 2}$ cells, including VEGF-A, VEGF-C and VEGF-D, were measured using qPCR assay. No significant difference at the mRNA levels of VEGF-A and VEGF-C was detected between $\mathrm{H} 520^{\mathrm{CTRL}}$ and $\mathrm{H} 520^{\mathrm{OV}-\mathrm{VASH}}{ }^{2}$ cells. But mRNA and protein level of VEGF-D in $\mathrm{H} 520^{\mathrm{OV}-\mathrm{VASH} 2}$ cells was significantly higher than that in $\mathrm{H} 520^{\mathrm{CTRL}}$ cells $(p=0.008, p=0.046$, Figure $3 A-B)$. The level of VEGF-D protein in the supernatants of H520 ${ }^{\mathrm{OV}-\mathrm{VASH} 2}$ and H520 ${ }^{\mathrm{CTRL}}$ was compared by ELISA assay. We found H520 OV-VASH2 secreted more VEGF-D than H520 CTRL $(497.37 \pm 67.51 \mathrm{pg} / \mathrm{ml}$ vs. $218.54 \pm 45.36 \mathrm{pg} / \mathrm{ml}, \mathrm{p}=0.023$, Figure $3 \mathrm{C})$. Furthermore, specific VEGF-D blocking antibody could efficiently inhibited the tube formation of HLECs co-cultured with $\mathrm{H} 520^{\mathrm{OV}-\mathrm{VASH} 2}$ $(29.67 \pm 4.67$ vs. $64.48 \pm 8.12, p=0.003$, Figure 3D). These data implied that VASH2 over-expression promoted the production and secretion of VEGF-D in LUSC cells, which might directly facilitate VASH2indiced lymphangiogenesis in vitro. VEGF-D antibody was added to the two groups of cells respectively. The effect of VEGF-D on VASH2 production and release was detected by ELISA and PCR. The level of VASH2 protein in the supernatants has no difference in $\mathrm{H} 520^{\mathrm{OV}-V A S H 2+V E G F-D A B}$ compared with $\mathrm{H} 520^{\mathrm{OV}}$ $\operatorname{VASH} 2(563.54 \pm 89.21 \mathrm{pg} / \mathrm{ml}$ vs $592.72 \pm 60.34 \mathrm{pg} / \mathrm{ml}, \mathrm{p}=0.24$, Figure $3 E)$. There is still no difference in mRNA expression (6.83 \pm 1.64 vs $8.61 \pm 1.51, p=0.24$, Figure $3 F)$.

\section{VASH2 enhanced tumor proliferation and invasion via up-regulating Snail expression in LUSC cells}

The transcription of multiple EMT-related biomarkers was detected using qPCR assay, and we found that the level of Snail mRNA increased in $\mathrm{H} 520^{\mathrm{OV}-\mathrm{VASH} 2}$ compared to $\mathrm{H} 520^{\mathrm{CTRL}}$ but that of E-cadherin decreased which indicated VASH2 promoting EMT in LUSC cells. However, there was no significant difference of the mRNA levels of the mesenchymal cell biomarkers $\beta$-catenin and $N$-cadherin, and other EMT-related TFs, such as Zeb family (Zeb1 and Zeb2), Slug, and Twist1 (Figure 4A). These results implied that VASH2 promoted LUSC cell EMT by stimulating Snail transcription.

To further determine if VASH2 up-regulated LUSC cell proliferation and invasion via Snail, we further designed a recombinant lentivirus to knock down Snail in H520 OV-VASH2 cells (H520 OV-VASH2+sh-Snail), as well as another recombinant lentivirus to force over-expression of Snail in wide-type H520 cells to establish H520 OV-Snail (Figure S2B-C). We compared the proliferation of $\mathrm{H} 520^{\mathrm{CTRL}}$, H520 OV-VASH2, $\mathrm{H} 520^{\mathrm{OV}-}$ VASH2+sh-Snail and $\mathrm{H} 520^{\mathrm{OV}-S n a i l}$ cells using the CCK-8 staining assay and found that the proliferation rates were lower in $\mathrm{H}_{520} \mathrm{OV}-\mathrm{VASH} 2+$ sh-Snail than that in $\mathrm{H} 520^{\mathrm{OV}-\mathrm{VASH} 2}$ (H520 OV-VASH2+sh-Snail: 2.51 \pm 0.38 ; H520 OVVASH2: $3.97 \pm 0.52$; H520 CTRL: $2.07 \pm 0.31, \mathrm{p}=0.022$, Figure 4B). And the proliferation rates were higher in $\mathrm{H} 520^{\mathrm{OV} \text {-Snail }}$ than that in $\mathrm{H} 520^{\mathrm{CTRL}}$ ( $4.14 \pm 0.52$ vs. $2.36 \pm 0.38, \mathrm{p}=0.031$, Figure $4 \mathrm{C}$ ). The cell migration and invasion were compared using the wound healing and trans-well assays. It showed that less $\mathrm{H} 520^{\mathrm{OV}}$ VASH2+sh-Snail and more $\mathrm{H} 520^{\mathrm{OV}-S n a i l}$ migrated across the Matrigel layer compared with their control cells (H520 OV-VASH2+sh-Snail: 175.36 \pm 23.47 ; H520 OV-VASH2: 254.17 \pm 30.78 ; H520 CTRL: $164.24 \pm 20.14, p=0.024$, Figure 4D; $217.93 \pm 16.77$ vs. $125.69 \pm 15.13, p=0.002$, Figure $4 E$ ). The similar results were detected in

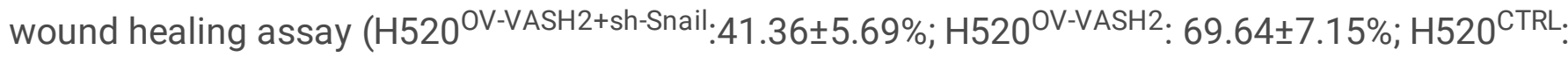


$37.25 \pm 4.95 \%, p=0.005$, Figure 4F; $85.32 \pm 8.47 \%$ vs. $34.25 \pm 5.68, p=0.001$, Figure 4 G). These results demonstrated that VASH2 promoted the proliferation and invasion of LUSC cells via up-regulating Snail transcription and expression.

\section{Snail regulated VASH2-related lymphangiogenesis via stimulating VEGF-D production in LUSC cells}

We found that VEGF-D was also up-regulated in samples with high expression of Snail in $\mathrm{H} 520^{\mathrm{OV}-\mathrm{VASH} 2}$ which implied a potential causal relationship between Snail and VEGF-D. Therefore, we first determined if Snail mediated VASH2-induced lymphangiogenesis. We transfected $\mathrm{H} 520^{\mathrm{OV}-\mathrm{VASH} 2}$ cells with sh-Snail recombinant lentivirus and compared the tube formation capacity of HLECs or HUVECs co-cultured with either $\mathrm{H}_{52} \mathrm{C}^{\mathrm{CTRL}}, \mathrm{H} 520^{\mathrm{OV}-\mathrm{VASH}} 2$, H520 OV-VASH2+sh-Snail or H520 OV-Snail cells. The number of HLEC-formed tubes in $\mathrm{H} 520^{\mathrm{OV}-\mathrm{VASH} 2+\text { sh-Snail }}$ group was less than that in $\mathrm{H} 520^{\mathrm{OV}-\mathrm{VASH} 2}$ group $(40.67 \pm 6.43 \% \mathrm{vs}$. $64.67 \pm 7.67 \%, p=0.014$, Figure $5 A)$. However, there was no difference between the number of HUVECformed tubes in $\mathrm{H} 520^{\mathrm{OV}-\mathrm{VASH} 2}$ and H520 ${ }^{\mathrm{OV}-\mathrm{VASH} 2+\text { sh-Snail }}$ groups $(28.54 \pm 3.64 \%$ vs. $37.64 \pm 5.79 \%, \mathrm{p}=0.083$, Figure 5B). Furthermore, when we transfected H520 cells with Snail recombinant lentivirus, and found that the number of $\mathrm{HLEC}$-formed tubes in $\mathrm{H} 520^{\mathrm{OV}}$-Snail group was higher than that in $\mathrm{H} 520^{\mathrm{CTRL}}$ group $(85.32 \pm 8.47 \%$ vs. $34.25 \pm 5.68 \%, p=0.001$, Figure $5 C$ ). There is still no difference in the HUVEC-formed tubes $(20.98 \pm 4.23 \%$ vs. $27.36 \pm 6.71 \%, p=0.24$, Figure 5D).

In order to determine if Snail regulated VASH2-stimulated VEGF-D production, H520 CTRL, H520 OV-VASH2, $\mathrm{H} 520^{\mathrm{OV}-\mathrm{VASH} 2+\text { sh-Snail }}$ and H520 ${ }^{\mathrm{OV}-S n a i l}$ cells were collected to compare the mRNA levels of VEGF-A, VEGF$\mathrm{C}$ and VEGF-D at by qPCR assay. Compared to H520 ${ }^{\mathrm{OV}-\mathrm{VASH} 2}$, a significant decrease of the mRNA level of VEGF-Din H520 OV-VASH2+sh-Snail, but no difference was detected in the mRNA levels of VEGF-A and VEGF-C (Figure 5E). Consistently, compared to $\mathrm{H} 520^{\mathrm{CTRL}}$, over-expression of Snail induced a significant increase of VEGF-D at 6.47 folds rather than VEGF-A and VEGF-C ( $p=0.008$, Figure 5F). Above results indicated that Snail regulated $\mathrm{VASH} 2$-related lymphangiogenesis rather than angiogenesis via stimulating VEGF-D production.

\section{VASH2 was significantly correlated with either Snail or VEGF-D in primary LUSC tissues}

The protein levels of Snail and VEGF-D in 64 cases of primary LUSC tissues were assessed using IHC staining assay (Figure $6 \mathrm{~A}$ ). We used qPCR to detect the mRNA levels of VASH2, Snail and VEGF-D in LUSC. Snail and VEGF-D were highly expressed in VASH2 overexpression samples, and VEGF-D was also highly expressed in snail overexpression samples (Figure 6B). In addition, the results of IHC were consistent with this result. The positive rate and intensity of Snail inVASH2-positiveLUSC tissues were higher than that in VASH2-negativeLUSC tissues $(73.92 \pm 2.43 \%$ vs. $20.16 \pm 2.72 \%, p=0.019$, Figure $6 \mathrm{C})$. The similar results were observed in the average positive rate of VEGF-D $(67.06 \pm 3.85 \%$ vs. $25.08 \pm 3.36 \%$, $\mathrm{p}=0.035$, Figure $6 \mathrm{C}$ ). And among the Snail-positive LUSC patients, the average positive rate of VEGF-D was much higher than that in Snail-negative LUSC patients $(61.74 \pm 4.19 \%$ vs. $20.98 \pm 2.87 \%, p=0.033$, Figure 6C). 
To further compare the correlation amongVAHS2/Snail/VEGF-D and prognosis of patients with LUSC, we divided them into double positive group. We compared the OS between $\mathrm{VASH} 2^{+}$Snail ${ }^{+} \mathrm{LUSC}$ patients and non-VASH $2^{+}$Snail ${ }^{+}$patients, as well as between VASH2 ${ }^{+}$VEGF-D ${ }^{+}$patients and non-VASH2 ${ }^{+}$VEGF-

$\mathrm{D}^{+}$patients. Kaplan-Meier survival analysis revealed that $\mathrm{VASH}^{+}{ }^{+}$Snail ${ }^{+}$patients suffered from shorter OS than non-VASH2 ${ }^{+}$Snail ${ }^{+}$patients $(17.21 \pm 1.39$ months vs. $70.75 \pm 2.54$ months, $p=0.062$, Figure 6D). Similarly, VASH2 ${ }^{+}$VEGF-D ${ }^{+}$patients exhibited a shorter OS than non-VASH2 ${ }^{+}$VEGF-D ${ }^{+}$patients $(39.93 \pm$ 5.46 months vs. $63.71 \pm 3.27$ months, $p=0.011$, Figure 6D).

\section{VASH2 blocking antibody inhibited tumor growth and lymphangiogenesis by attenuating VEGF-D production}

$\mathrm{H} 520^{\mathrm{CTRL}}$ and $\mathrm{H} 520^{\mathrm{OV}-\mathrm{VASH}} 2$ were injected at $1 \times 10^{6}$ tumor cells $/ 100 \mu \mathrm{l}$ per mouse subcutaneously into NOD-SCID mice. After 26 days, the average volumes of tumors generated in H520 OV-VASH2 group were nearly 3-fold larger than those in H520 ${ }^{\mathrm{CTRL}}$ group $\left(1805.62 \pm 171.56 \mathrm{~mm}^{3}\right.$ vs. $648.64 \pm 92.21 \mathrm{~mm}^{3}, \mathrm{p}=0.041$, Figure 7A). The proportion of LYVE-1 positive cells referring to lymphoepithelial cells in $\mathrm{H} 520^{\mathrm{OV}-\mathrm{VASH} 2}$ was significantly higher than $\mathrm{H} 520^{\mathrm{CTRL}}(75.37 \pm 8.63 \%$ vs. $4.26 \pm 0.74 \%, \mathrm{p}=0.001$, Figure $7 \mathrm{~B})$, which indicated that VASH2 promoted the lymphangiogenesis in vivo.

In order to confirm if targeted therapy against either VASH2 or VEGF-D was efficient to inhibit tumor metastasis and local lymphangiogenesis in vivo, mice were treated with anti-VASH2 or anti-VEGF-D antibodies at a dose of $2.5 \mathrm{mg} / \mathrm{kg}$ bodyweight by i.p. injection three times per week. We found the tumor sizes were significantly reduced after either treatment (H520 OV-VASH2: $1805.62 \pm 171.56 \mathrm{~mm}^{3} ; \mathrm{H} 520^{\mathrm{OV}}$ VASH2+VASH2AB: $1105.36 \pm 92.21 \mathrm{~mm}^{3} ; \mathrm{H}_{520}$ OV-VASH2+VEGF-DAB: $1281.36 \pm 215.36 \mathrm{~mm}^{3}, \mathrm{p}<0.01$, Figure 7A). Furthermore, after anti-VASH2 or anti-VEGF-D antibodies, the proportion of lymphoendothelial cells was

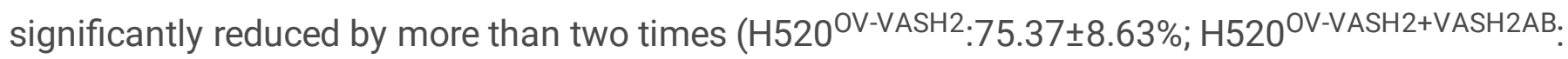
$31.86 \pm 4.14 \%$; H520 OV-VASH2+VEGF-DAB: $26.73 \pm 3.27 \%, \mathrm{p}<0.01$, Figure 7B). The similar results were observed by flow cytometry by labeling D2-40 positive cells, which is another marker of lymphoepithelial cells (H520 OV-VASH2: 79.71 $\pm 9.58 \%$; H520 CTRL: $32.48 \pm 4.36 \%$; H520VASH2-VASH2Ab: $44.82 \pm 5.36 \%$; H520 OVVASH2+VEGF-DAB: $47.42 \pm 5.69 \%, \mathrm{p}<0.01$, Figure $7 C$ ).

Then, we extracted tumor RNA and compared the mRNA levels of Snail and VEGF-D between different groups using qPCR assay. We noticed that the mRNA levels of above genes were significantly upregulated in $\mathrm{H} 520^{\mathrm{OV}-\mathrm{VASH} 2}$ compared to $\mathrm{H} 520^{\mathrm{CTRL}}$, which was consistent with the observation in vitro. (Snail: $2.89 \pm 0.36, p=0.009$; VEGF-D: $4.97 \pm 0.68, p=0.007$, Figure 7D-E). We also noticed that the mRNA levels of Snail and VEGF-D were significantly down-regulated in $\mathrm{H} 520^{\mathrm{OV}-\mathrm{VASH} 2+\mathrm{VASH} 2 \mathrm{AB}}$ compared to H520 OV-VASH2 (Snail: $0.79 \pm 0.11$ vs $2.89 \pm 0.36, p=0.006$; VEGF-D: $1.75 \pm 0.84$ vs $4.97 \pm 0.68, p=0.006$, Figure 7D-E). But the mRNA expression of Snail has no difference in $\mathrm{H} 520^{\mathrm{OV}-\mathrm{VASH} 2+\mathrm{VEGF}-\mathrm{DAB}}$ compared with H520 OV-VASH2 (Snail: $2.48 \pm 0.19$ vs $2.89 \pm 0.36, p=0.15$; VEGF-D: $2.12 \pm 0.98$ vs $4.97 \pm 0.68, p=0.014$, Figure 7D-E). The same conclusion could be reached by detecting protein level by western blot. The protein 


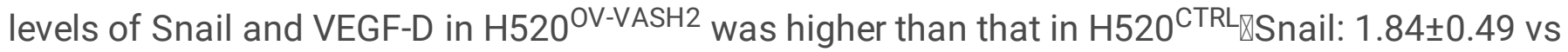
$1.13 \pm 0.095, p=0.044$; VEGF-D: $1.49 \pm 0.64$ vs $0.92 \pm 0.12, p=0.026$, Figure 7D-E). The protein levels of Snail and VEGF-D were significantly decreased by adding VASH2 antibody (Snail: $0.57 \pm 0.094$ vs $1.84 \pm 0.49$, $\mathrm{p}=0.012$; VEGF-D: $0.89 \pm 0.084$ vs $1.49 \pm 0.64, \mathrm{p}=0.017$, Figure 7D-E). When VEGF-D antibody was added, the protein level of Snail had no difference (Snail: $1.52 \pm 0.28$ vs $1.84 \pm 0.49, p=0.38$; VEGF-D: $0.78 \pm 0.19$ vs $1.49 \pm 0.64, p=0.013$, Figure 7D-E).

\section{Discussion}

Although many studies have confirmed the predominant role of microvascular development, especially angiogenesis in tumor growth and metastasis, the anti-angiogenesis therapy has not achieved sufficient clinical efficacy as expected until now [21]. Actually, recent studies showed that the initial metastasis of most malignant tumors is not through blood vessels, but firstly through lymphatic vessels to local lymph nodes $[22,23]$. Lymphangiogenesis, the development of new lymph vessels, has been reported to promote lymphatic invasion and lymph node metastasis. Therefore, anti-lymphangiogenesis therapy has become a relatively hot topic of clinical investigation and might be more promising strategy to inhibit the metastasis and spread of tumors in early stage.

In this study, based on 937 LUSC gene expression profiling data from 11 public datasets in GEO and TCGA dataset, as well as 64 LUSC primary tissues from TJMUCH independent cohort, we demonstrated that VASH2 was an independent prognostic biomarker for LUSC. We validated the positive correlation between VASH2 and poor prognosis of LUSC patients both at mRNA and protein levels. The IHC results of an independent Chinese LUSC cohort showed that there was no correlation between the expression of VASH2 and CD31 and other vascular endothelial markers, while the pathological analysis showed that the expression of VASH2 was significantly related to the number of lymph node metastasis in LUSC patients. Furthermore, we found that VASH2 expression was significantly positively correlated with the number of lymph node metastasis rather than distant metastasis. We indicated that VASH2 promoted amplification and tube-formation of HUVEC and HLEC cells, and accelerated proliferation and invasion of LUSC cells. Further studies demonstrated that VASH2 facilitated lymphangiogenesis via up-regulating VEGF-D production in LUSC cells and enhanced tumor proliferation and invasion via up-regulating Snail expression in LUSC cells.

VASH2 was reported as an angiogenic factor promoting tumor development and progression [13, 24]. VASH2 regulates the expression of VEGF through autocrine and paracrine mechanisms, and promotes angiogenesis [25]. VEGF family was identified as an endothelial cell-specific mitogen that has the capacity to induce physiological and pathological angiogenesis and lymphangiogenesis. Among the VEGF family members, VEGF-C and VEGF-D have similar structures, but bind and activate VEGF receptor VEGFR-2 and VEGFR-3, respectively. VEGF-C and VEGF-D promoted angiogenesis and lymphangiogenesis within and at the periphery of solid tumors, as well as enhancing solid tumor growth and metastatic spread to lymph nodes and distant organs $[26,27]$. Actually, the understanding of the molecular mechanisms of VEGF-C in lymphatic development and lymphangiogenesis has been greatly appreciated 
in multiple cancers, however, the role of VEGF-D is less well understood. Previous studies showed that VEGF-D could compensate for the loss of VEGF-C for the sprouting of lymphatics, indicating VEGF-D might play a subtler role in regulating lymphatic vascular development than VEGF-C [28-30]. In this study, we found VEGF-D was significantly up-regulated in H520OV-VASH2 cells, but the expression of VEGF-C did not change significantly, suggesting that VASH2 promoted lymphangiogenesis mainly through upregulation of VEGF-D, but not VEGF-C signaling.

Snail has been reported to promote lymph node metastasis [31]. Snail is a transcription factor involved in EMT, but plays an important role in lymphatic metastasis, and increased lymph node metastasis. Consistently, we found that Snail positively correlated with the level of VASH2 and the status of lymph node metastasis.

The relationship between Snail and lymphangiogenesis, as well as Snail and VEGF-D, are not fully investigated yet [32].

In this study, we found that Snail participated in VASH2-mediated lymphangiogenesis via up-regulation of VEGF-D in LUSC cells. Inhibition VEGF-D via anti-VEGF-D antibody could reduce tumor growth in vivo, not affecting Snail expression, which implied a "VASH2-Snail-VEGF-D" axis in promoting lymphangiogenesis and tumor progression in LUSC. Furthermore, we observed that inhibition of VASH2 showed better therapy effect than inhibition of VEGF-D in the mouse model, which is most likely due to the function of VASH2 monoclonal antibodies in interfering proliferation of cancer cells as well as lymphangiogenesis in tumor tissues simultaneously.

\section{Conclusion}

In conclusion, we found that the high expression of VASH2 was significantly related to the poor prognosis and lymph node metastasis of LUSC patients. VASH2 could increase the expression of VEGF-D, upregulate the expression of Snail and down-regulate the expression of E-cadherin in LUSC cells, and promote the formation of lymphatics. Therefore, further investigations for a comprehensive understanding of VASH2 in tumor development will probably provide us more effective promising therapeutic strategies for treating metastatic disease.

\section{Abbreviations}

LUSC lung squamous cell carcinoma

TCGA The Cancer Genome Atlas

GEO Gene Expression Omnibus

VASH2 Vasohibin 2

HUVECs human umbilical vein endothelial cells 
HLECs human lymphatic endothelial cells

VEGF-D Vascular endothelial growth factor-D

LUAD lung adenocarcinoma

MNCs monocytes

EMT epithelial-mesenchymal transition

OS overall survival

IHC immunohistochemical

qPCR quantitative PCR

WCRs wound closure rates

ELISA Enzyme-linked immunosorbent assay

CCK-8 Cell counting kit-8

\section{Declarations}

\section{Ethics approval and consent to participate}

This study was approved by the Ethics Committee of Tianjin Medical University. All experiments were performed in accordance with the principles of the Declaration of Helsinki and written consent was obtained from all patients.

\section{Consent for publication}

My material is original research, has not been previously published and has not been submitted for publication elsewhere while under consideration.

\section{Availability of data and material}

Not applicable

\section{Competing interests}

No conflict of interest exits in the submission of this manuscript, and manuscript is approved by all authors for publication. The work described was original research that has not been published previously, and not under consideration for publication elsewhere, in whole or in part. Consent for publication was obtained from all patients contained in our manuscript. 


\section{Funding}

This work was supported by National Natural Science Foundation of China (grant numbers: 82072588 , $82002601,81872143,81702280)$. National Science and Technology support Program of China (Grant No. 2018ZX09201015), Projects of Science and Technology of Tianjin (Grant No. 18JCQNJC82700) and Key project of Tianjin Health and Family Planning Commission (Grant No. 16KG126).

\section{Authors' contributions}

PPL performed data analyses, and wrote the manuscript. PPL, RZ and XZ performed the research jointly. LH performed bioinformatics analysis. YNY and XBR contributed clinical information and samples for the study. WZ gave guidance and help on the bioinformatics analysis. JPY designed the study and commented on manuscript.

\section{References}

1. Rafiemanesh H, Mehtarpour M, Khani F, Hesami SM, Shamlou R, Towhidi F, et al. Epidemiology, incidence and mortality of lung cancer and their relationship with the development index in the world. J Thorac Dis. 2016;8(6):1094-102.

2. Zakaria N, Yusoff NM, Zakaria Z, Lim MN, Baharuddin PJN, Fakiruddin KS, et al. Human non-small cell lung cancer expresses putative cancer stem cell markers and exhibits the transcriptomic profile of multipotent cells. BMC Cancer. 2015;15:84-

3. Jemal A, Siegel R, Ward E, Hao Y, Xu J, Thun MJ. Cancer statistics, 2009. CA: a cancer journal for clinicians. 2009;59(4):225-49.

4. Patz EF, Jr., Greco E, Gatsonis C, Pinsky P, Kramer BS, Aberle DR. Lung cancer incidence and mortality in National Lung Screening Trial participants who underwent low-dose CT prevalence screening: a retrospective cohort analysis of a randomised, multicentre, diagnostic screening trial. Lancet Oncol. 2016;17(5):590-9.

5. Hu JM, Liu K, Liu JH, Jiang XL, Wang XL, Yang L, et al. The increased number of tumor-associated macrophage is associated with overexpression of VEGF-C, plays an important role in Kazakh ESCC invasion and metastasis. Experimental and Molecular Pathology. 2017;102(1):15-21.

6. Mumprecht V, Detmar M. Lymphangiogenesis and cancer metastasis. Journal of cellular and molecular medicine. 2009;13(8A):1405-16.

7. Li S, Li Q. Cancer stem cells, lymphangiogenesis, and lymphatic metastasis. Cancer letters. 2015;357(2):438-47.

8. Ikemura S, Aramaki N, Fujii S, Kirita K, Umemura S, Matsumoto S, et al. Changes in the tumor microenvironment during lymphatic metastasis of lung squamous cell carcinoma. Cancer Sci. 2017;108(1):136-42.

9. Moon Y, Park JK, Lee KY, Sung SW. Lymphatic invasion is a more significant prognostic factor than visceral pleural invasion in non-small cell lung cancer with tumours of $3 \mathrm{~cm}$ or less. Respirology. 
2017;22(6):1179-84.

10. Watabe T. Roles of transcriptional network during the formation of lymphatic vessels. The Journal of Biochemistry. 2012;152(3):213-20.

11. Sato Y. The vasohibin family: a novel family for angiogenesis regulation. J Biochem. 2013;153(1):511.

12. Koyanagi T, Suzuki Y, Komori K, Saga Y, Matsubara S, Fujiwara H, et al. Targeting human vasohibin-2 by a neutralizing monoclonal antibody for anti-cancer treatment. Cancer Sci. 2017;108(3):512-9.

13. Kitahara S, Suzuki Y, Morishima M, Yoshii A, Kikuta S, Shimizu K, et al. Vasohibin-2 modulates tumor onset in the gastrointestinal tract by normalizing tumor angiogenesis. Mol Cancer. 2014;13:99-.

14. Tu M, Lu C, Lv N, Wei J, Lu Z, Xi C, et al. Vasohibin 2 promotes human luminal breast cancer angiogenesis in a non-paracrine manner via transcriptional activation of fibroblast growth factor 2 . Cancer letters. 2016;383(2):272-81.

15. Takahashi Y, Koyanagi T, Suzuki Y, Saga Y, Kanomata N, Moriya T, et al. Vasohibin-2 expressed in human serous ovarian adenocarcinoma accelerates tumor growth by promoting angiogenesis. Molecular cancer research : MCR. 2012;10(9):1135-46.

16. Kimura H, Miyashita H, Suzuki Y, Kobayashi M, Watanabe K, Sonoda $H$, et al. Distinctive localization and opposed roles of vasohibin-1 and vasohibin-2 in the regulation of angiogenesis. Blood. 2009;113(19):4810-8.

17. Koyanagi T, Saga Y, Takahashi Y, Suzuki Y, Suzuki M, Sato Y. Downregulation of vasohibin-2, a novel angiogenesis regulator, suppresses tumor growth by inhibiting angiogenesis in endometrial cancer cells. Oncol Lett. 2013;5(3):1058-62.

18. Heishi T, Hosaka T, Suzuki Y, Miyashita H, Oike Y, Takahashi T, et al. Endogenous angiogenesis inhibitor vasohibin 1 exhibits broad-spectrum antilymphangiogenic activity and suppresses lymph node metastasis. Am J Pathol. 2010;176(4):1950-8.

19. Zhang Y, Xue X, Zhao X, Qin L, Shen Y, Dou H, et al. Vasohibin 2 promotes malignant behaviors of pancreatic cancer cells by inducing epithelial-mesenchymal transition via Hedgehog signaling pathway. Cancer Med. 2018;7(11):5567-76.

20. Xue X, Zhang Y, Zhi Q, Tu M, Xu Y, Sun J, et al. MiR200-upregulated Vasohibin 2 promotes the malignant transformation of tumors by inducing epithelial-mesenchymal transition in hepatocellular carcinoma. Cell Commun Signal. 2014;12:62-.

21. Yi M, Jiao D, Qin S, Chu Q, Wu K, Li A. Synergistic effect of immune checkpoint blockade and antiangiogenesis in cancer treatment. Mol Cancer. 2019;18(1):60-.

22. Pereira ER, Kedrin D, Seano G, Gautier O, Meijer EFJ, Jones D, et al. Lymph node metastases can invade local blood vessels, exit the node, and colonize distant organs in mice. Science. 2018;359(6382):1403-7.

23. Stewart CL, Warner S, Ito K, Raoof M, Wu GX, Kessler J, et al. Cytoreduction for colorectal metastases: liver, lung, peritoneum, lymph nodes, bone, brain. When does it palliate, prolong survival, and potentially cure? Curr Probl Surg. 2018;55(9):330-79. 
24. Norita R, Suzuki Y, Furutani Y, Takahashi K, Yoshimatsu Y, Podyma-Inoue KA, et al. Vasohibin-2 is required for epithelial-mesenchymal transition of ovarian cancer cells by modulating transforming growth factor- $\beta$ signaling. Cancer Sci. 2017;108(3):419-26.

25. Xue X, Gao W, Sun B, Xu Y, Han B, Wang F, et al. Vasohibin 2 is transcriptionally activated and promotes angiogenesis in hepatocellular carcinoma. Oncogene. 2013;32(13):1724-34.

26. Choi JU, Chung SW, Al-Hilal TA, Alam F, Park J, Mahmud F, et al. A heparin conjugate, LHbisD4, inhibits lymphangiogenesis and attenuates lymph node metastasis by blocking VEGF-C signaling pathway. Biomaterials. 2017;139:56-66.

27. Alishekevitz D, Gingis-Velitski S, Kaidar-Person O, Gutter-Kapon L, Scherer SD, Raviv Z, et al. Macrophage-Induced Lymphangiogenesis and Metastasis following Paclitaxel Chemotherapy Is Regulated by VEGFR3. Cell Rep. 2016;17(5):1344-56.

28. Miyata Y, Matsuo T, Ohba K, Mitsunari K, Sakai H. 1135 CIGARETTE SMOKING INDUCES LYMPHANGIOGENESIS, BUT NOT ANGIOGENESIS, AND VASCULAR ENDOTHELIAL GROWTH FACTOR-D, BUT NOT -A OR -C IN HUMAN BLADDER CANCER TISSUES. Journal of Urology. 2013;189(4):e463-e4.

29. Lin W, Jiang L, Chen Y, She F, Han S, Zhu J, et al. Vascular endothelial growth factor-D promotes growth, lymphangiogenesis and lymphatic metastasis in gallbladder cancer. Cancer letters. 2012;314(2):127-36.

30. Ebata R, Abe J, Yasukawa K, Hamada H, Higashi K, Suwazono Y, et al. Increased Production of Vascular Endothelial Growth Factor-D and Lymphangiogenesis in Acute Kawasaki Disease. Circulation Journal. 2011;75(6):1455-62.

31. Cho YA, Kim EK, Cho BC, Koh YW, Yoon SO. Twist and Snail/Slug Expression in Oropharyngeal Squamous Cell Carcinoma in Correlation With Lymph Node Metastasis. Anticancer research. 2019;39(11):6307-16.

32. Harris K, Gelberg HB, Kiupel M, Helfand SC. Immunohistochemical Features of EpithelialMesenchymal Transition in Feline Oral Squamous Cell Carcinoma. Veterinary Pathology. 2019;56(6):826-39.

\section{Tables}

Due to technical limitations, table 1-2 is only available as a download in the Supplemental Files section.

\section{Figures}



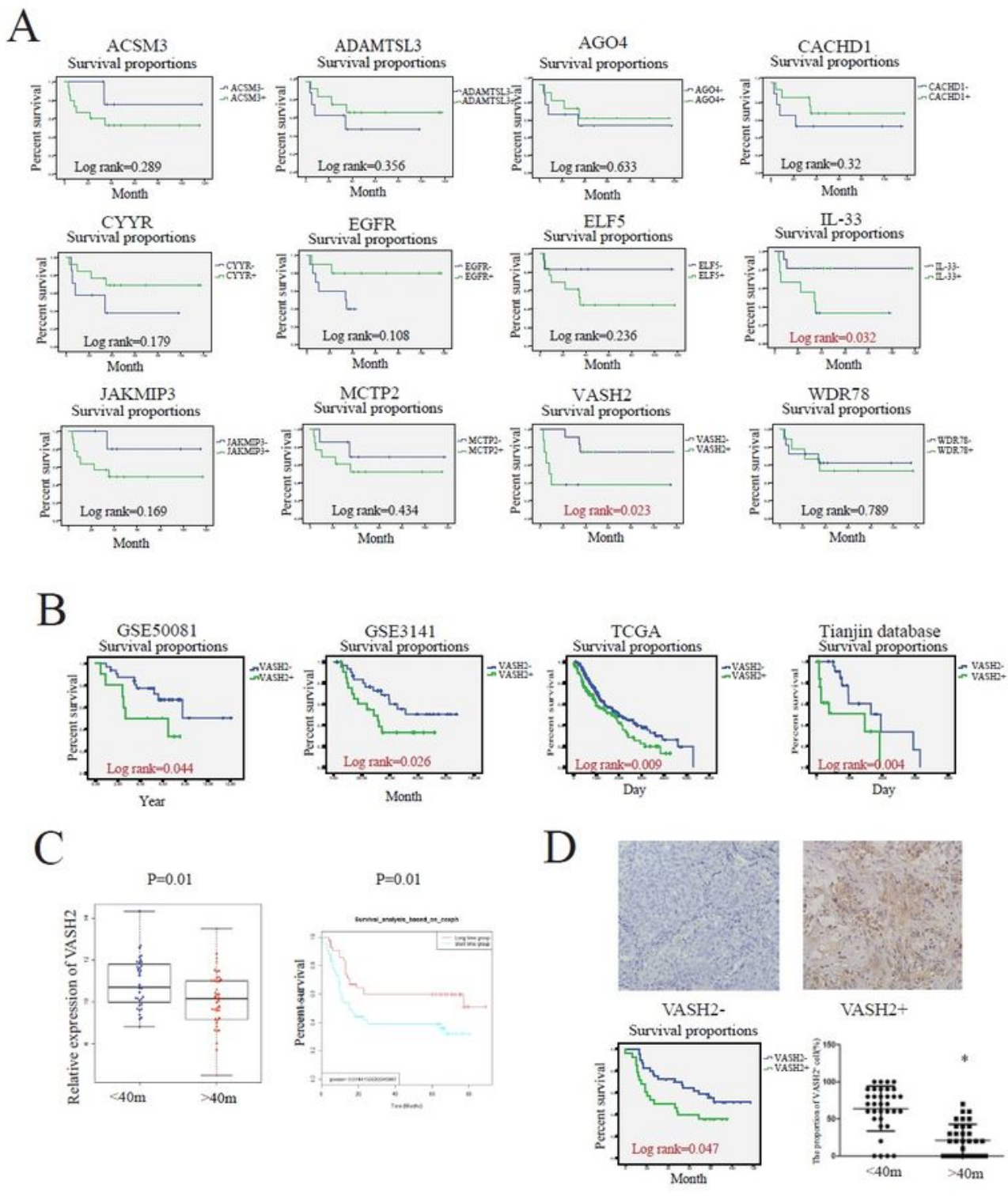

$\mathrm{E}$
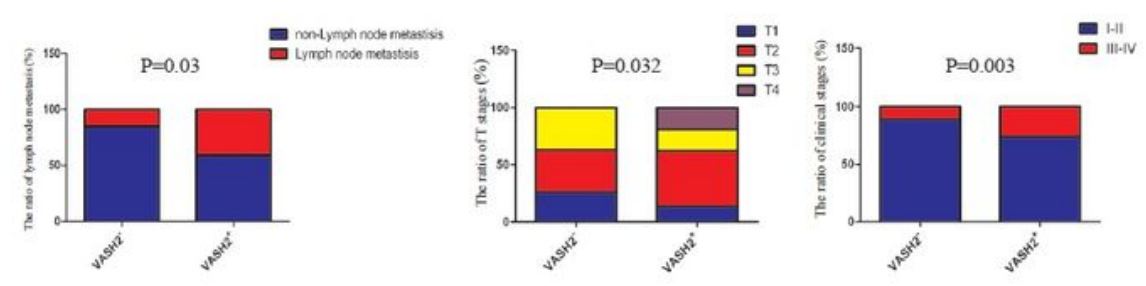

\section{Figure 1}

High expression of VASH2 correlated with worse prognosis and more lymphatic metastasis in LUSC patients A We collected LUSC tissue samples from TJMUCH to validate the correlation between the 12 identified genes and overall survival (OS) in 20 cases. B We collected and analyzed the global gene expression profiling of 937 primary LUSC tissue samples from 11 database based on the disparity in clinical outcomes. C We collected another independent cohort composing of 64 LUSC samples from 
TJMUCH which was detected using real-time quantitative PCR assay. D We compared the level of VASH2 protein in the corresponding LUSC paraffin specimens using IHC analysis. E we analyzed the association between VASH2 and other clinicopathology.

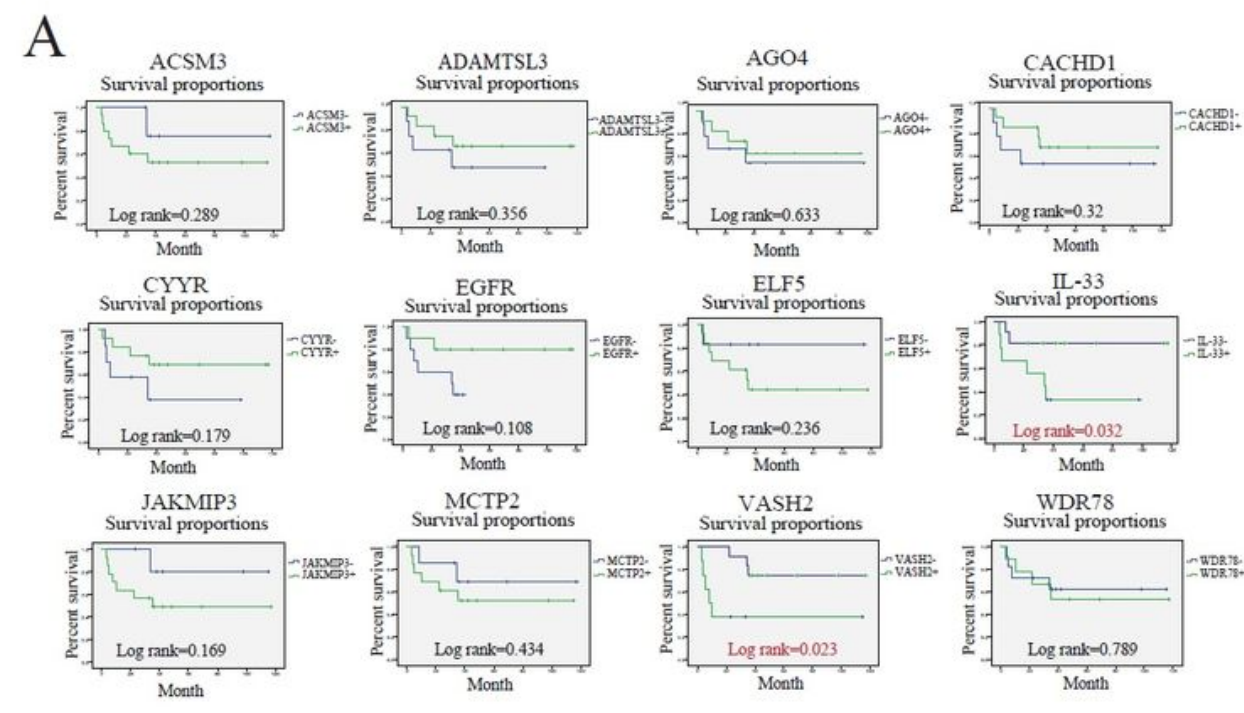

B
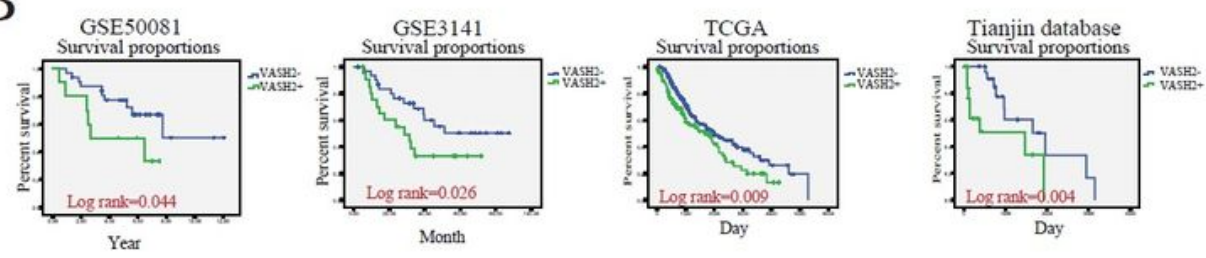

$\mathrm{C}$
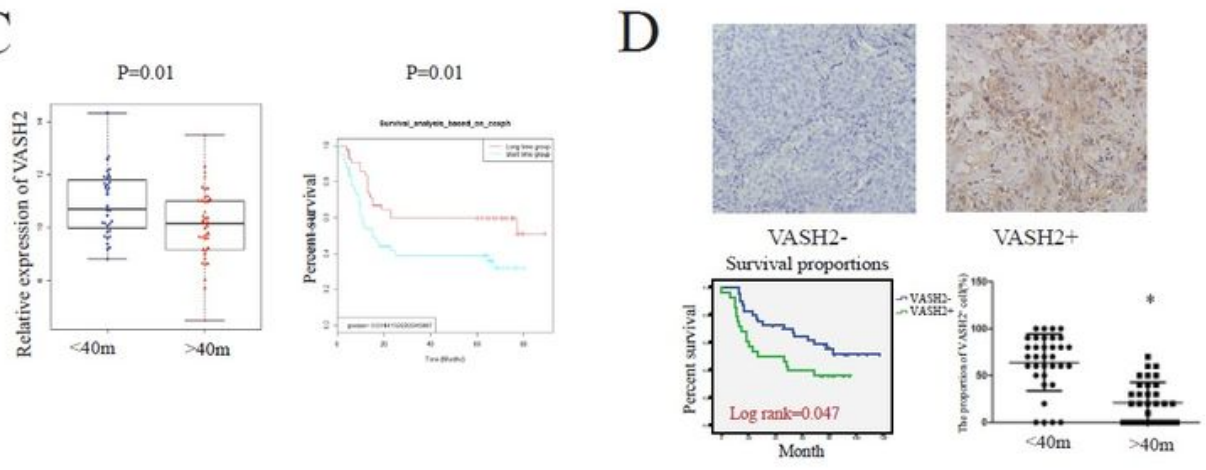

$\mathrm{E}$
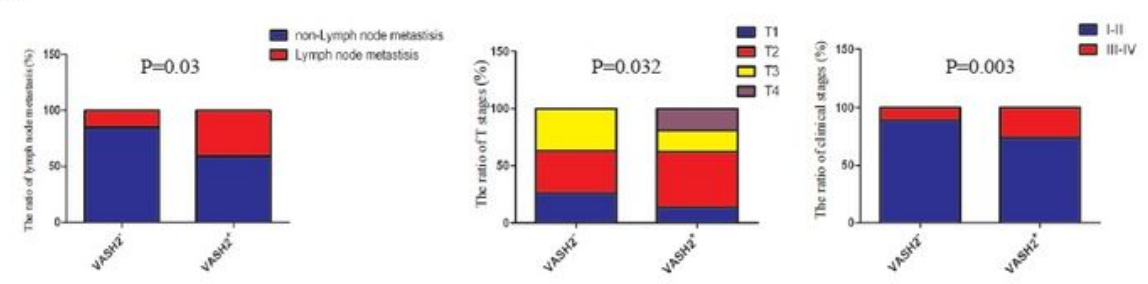

\section{Figure 1}

High expression of VASH2 correlated with worse prognosis and more lymphatic metastasis in LUSC patients A We collected LUSC tissue samples from TJMUCH to validate the correlation between the 12 
identified genes and overall survival (OS) in 20 cases. B We collected and analyzed the global gene expression profiling of 937 primary LUSC tissue samples from 11 database based on the disparity in clinical outcomes. C We collected another independent cohort composing of 64 LUSC samples from TJMUCH which was detected using real-time quantitative PCR assay. D We compared the level of VASH2 protein in the corresponding LUSC paraffin specimens using IHC analysis. E we analyzed the association between VASH2 and other clinicopathology.

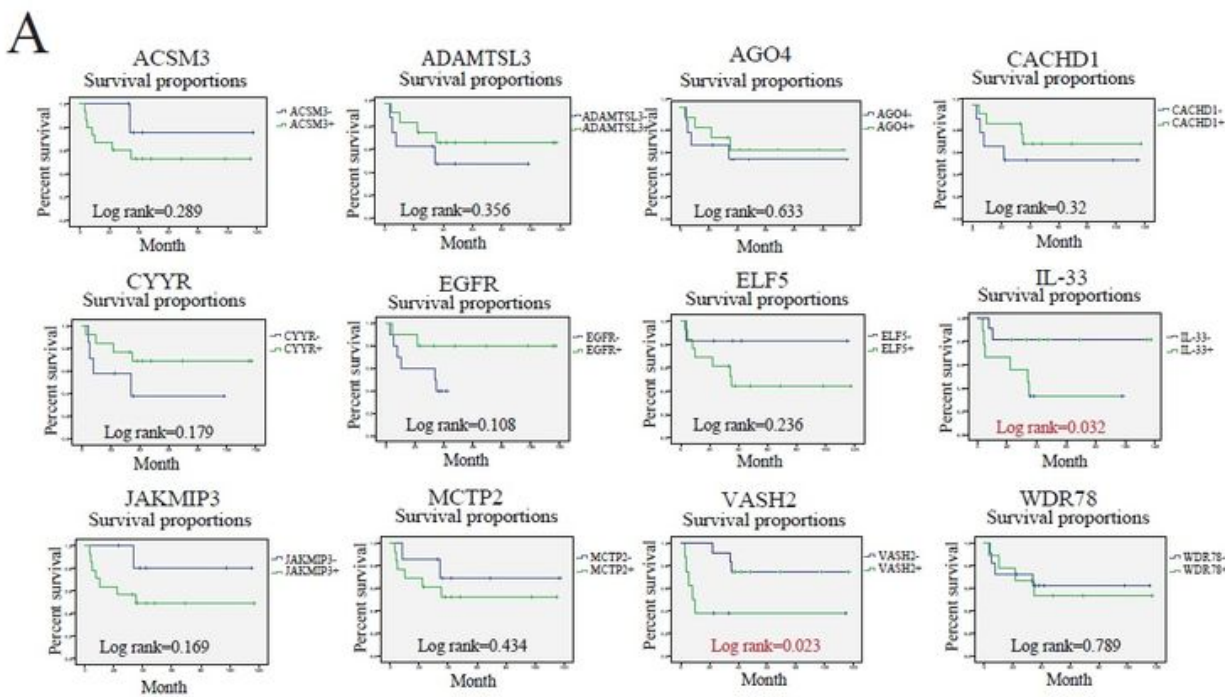

$\mathrm{B}$
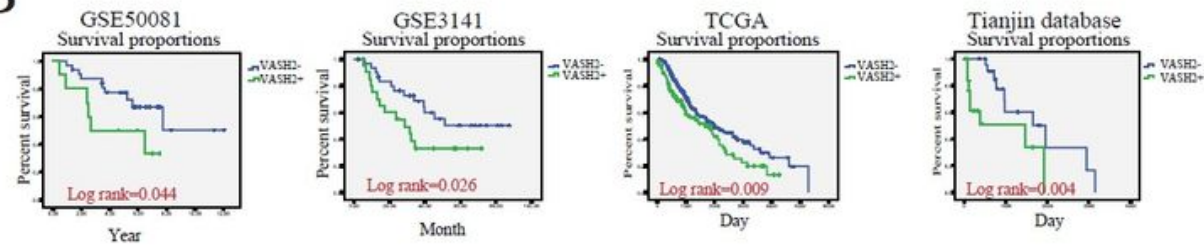

C
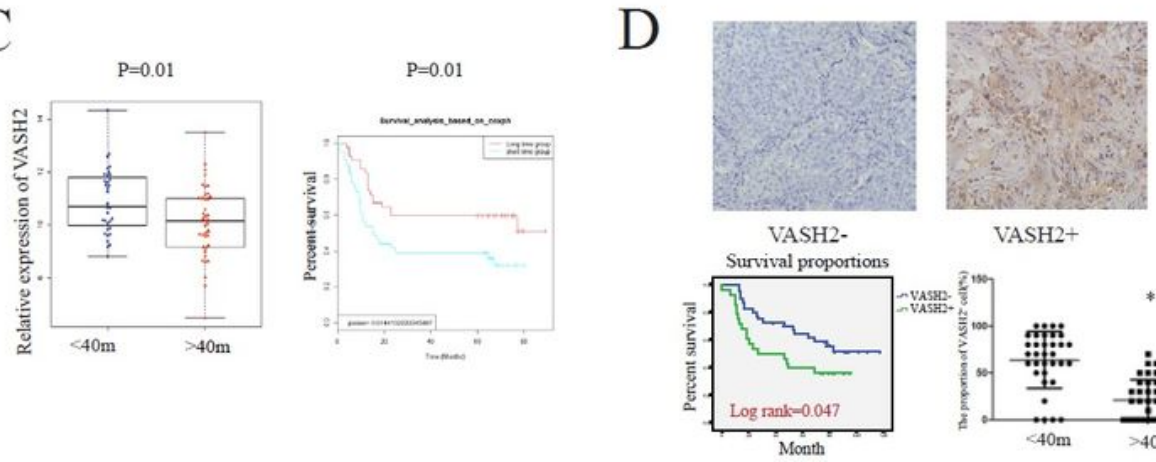

VASH2+

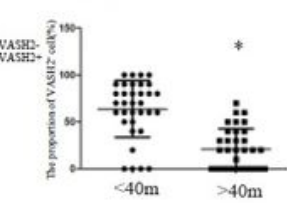

E
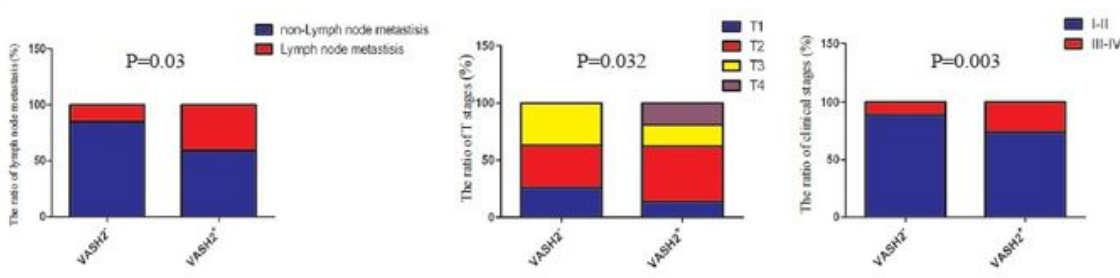

Figure 1 
High expression of VASH2 correlated with worse prognosis and more lymphatic metastasis in LUSC patients A We collected LUSC tissue samples from TJMUCH to validate the correlation between the 12 identified genes and overall survival (OS) in 20 cases. B We collected and analyzed the global gene expression profiling of 937 primary LUSC tissue samples from 11 database based on the disparity in clinical outcomes. C We collected another independent cohort composing of 64 LUSC samples from TJMUCH which was detected using real-time quantitative PCR assay. D We compared the level of VASH2 protein in the corresponding LUSC paraffin specimens using IHC analysis. E we analyzed the association between VASH2 and other clinicopathology. 

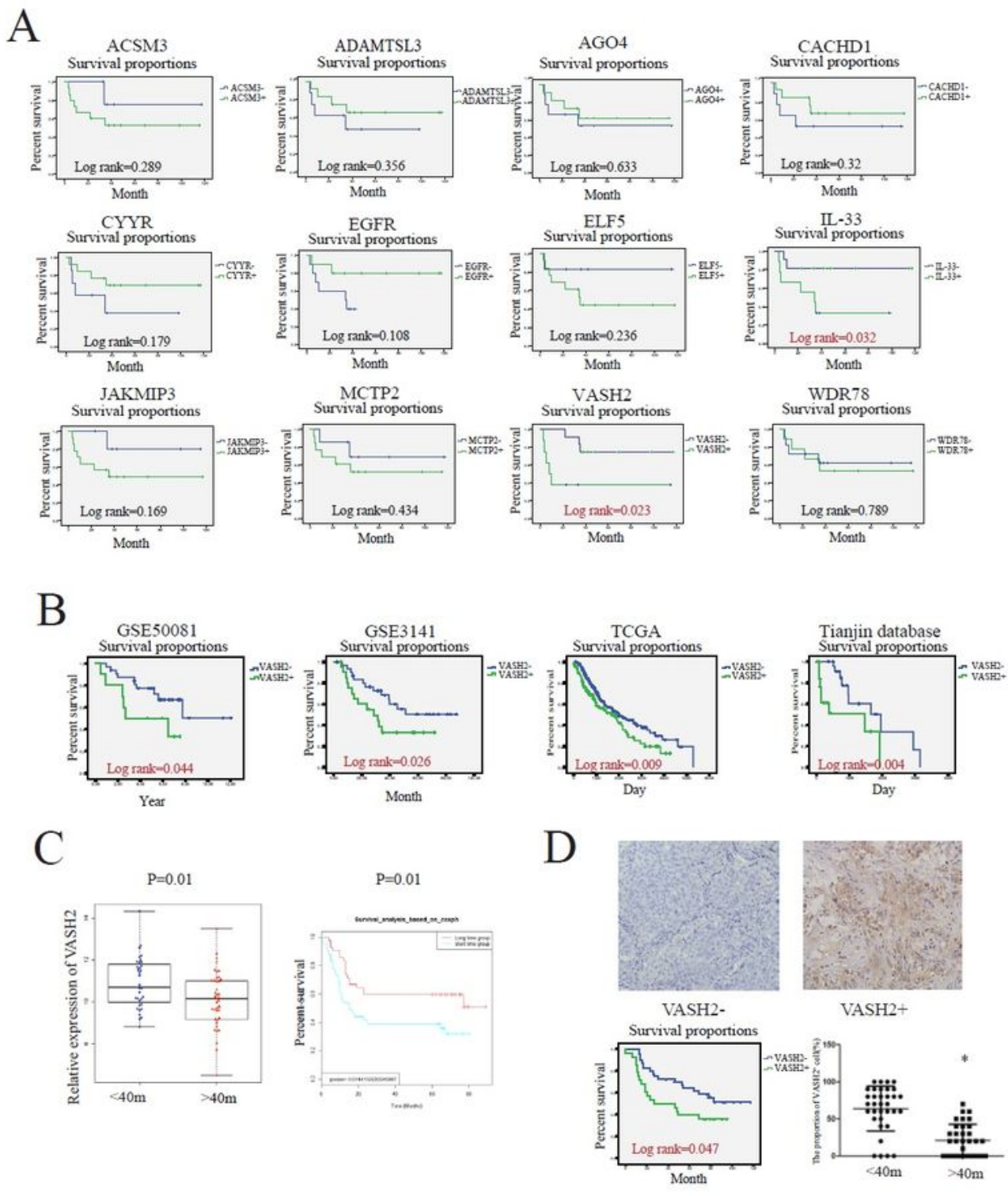

$\mathrm{E}$
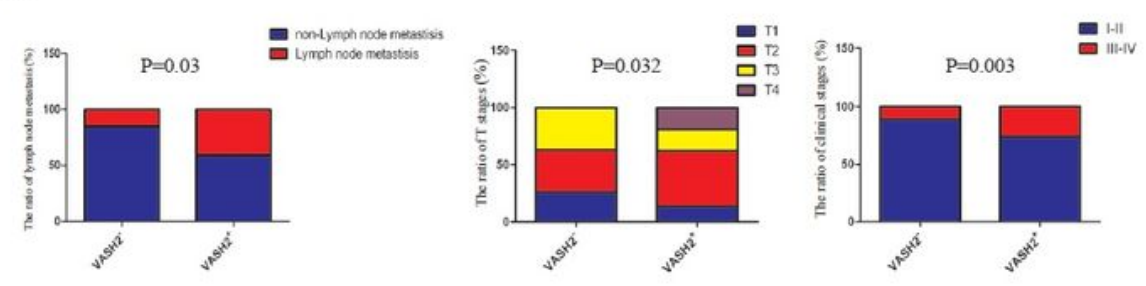

\section{Figure 1}

High expression of VASH2 correlated with worse prognosis and more lymphatic metastasis in LUSC patients A We collected LUSC tissue samples from TJMUCH to validate the correlation between the 12 identified genes and overall survival (OS) in 20 cases. B We collected and analyzed the global gene expression profiling of 937 primary LUSC tissue samples from 11 database based on the disparity in clinical outcomes. C We collected another independent cohort composing of 64 LUSC samples from 
TJMUCH which was detected using real-time quantitative PCR assay. D We compared the level of VASH2 protein in the corresponding LUSC paraffin specimens using IHC analysis. E we analyzed the association between VASH2 and other clinicopathology.

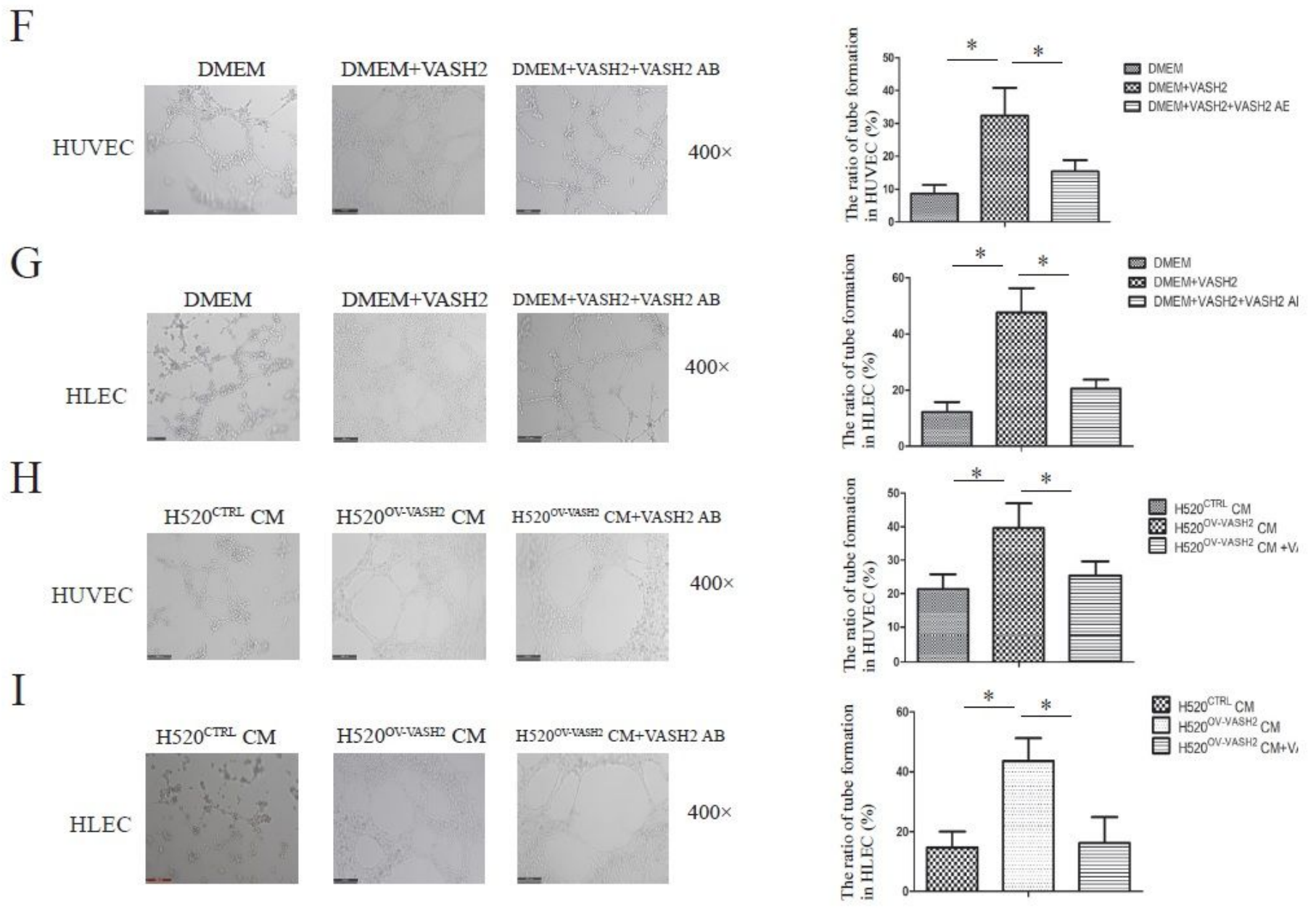

\section{Figure 2}

VASH2 accelerated the proliferation and invasion potential of LUSC cells, and promoted tube-formation capacity of HUVEC and HLEC cells A High level of VASH2 was confirmed in the supernatants of H5200VVASH2 compared to that in H520CTRL. The level of VASH2 protein in different supernatants was detected by ELISA. B We first compared the proliferation of H5200V-VASH2 by CCK-8 assay. C The apoptotic in H520CTRL and H5200V-VASH2 cells was measured using Annexin-V-FITC. D The migration and invasion potentials were compared by Wound Healing Assay. Images were taken at $0,24 \mathrm{~h}$ and $48 \mathrm{~h}$ later to measure the distance of the wound. E The Matrigel and Trans-well were used to carry out lung cancer cell invasion assays to build invading chambers for separating high invasive and low invasive cells. The stained membranes were photographed through the microscope and invading cells were counted after 48h. F, G, H, I HUVECs and HLECs were co-cultured with the supernatants from either H5200V-VASH2 orH520CTRL cells. The HUVECs and HLECs cultured in DMEM medium were used as negative controls. The numbers of forming tubes in each group were assessed. 
$\mathrm{F}$
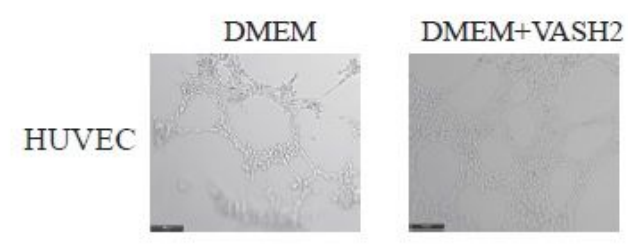

DMEM+VASH2+VASH2 AB

G
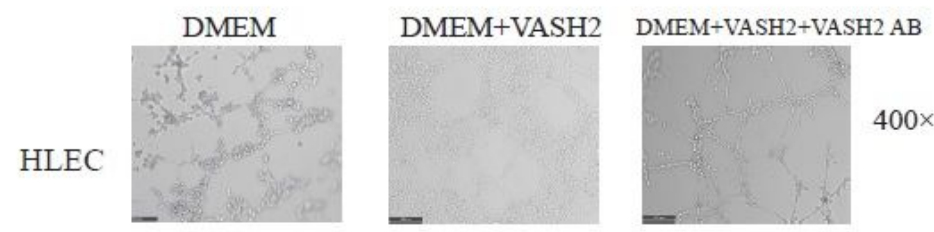

$\mathrm{H}$

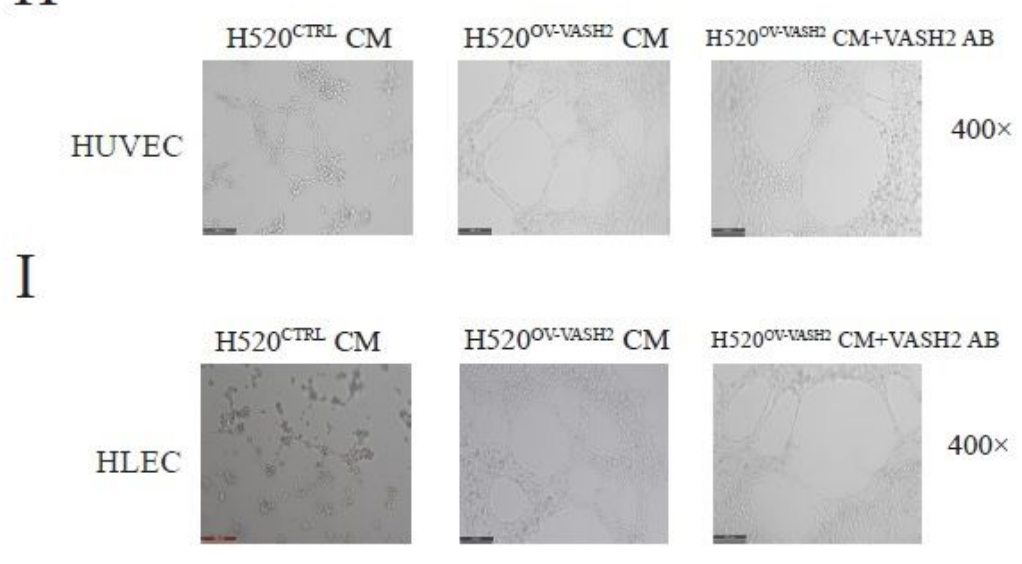

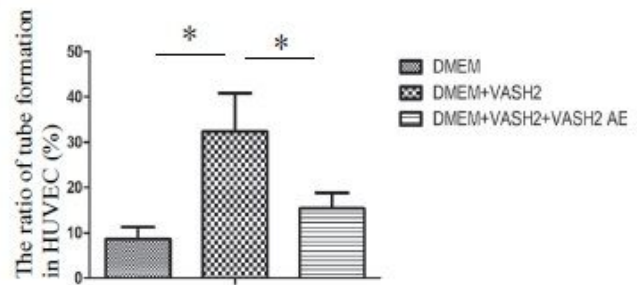
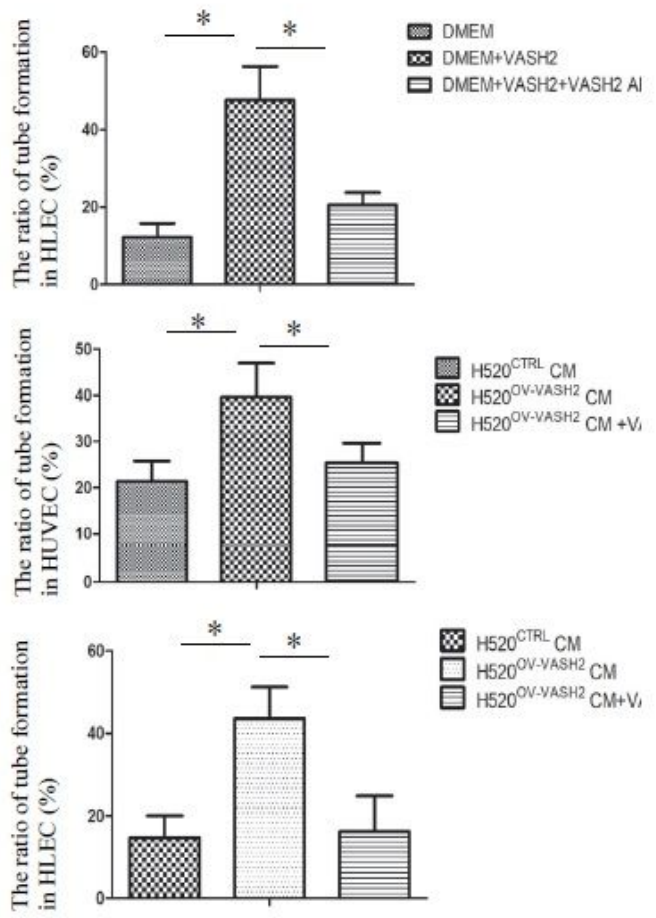

\section{Figure 2}

VASH2 accelerated the proliferation and invasion potential of LUSC cells, and promoted tube-formation capacity of HUVEC and HLEC cells A High level of VASH2 was confirmed in the supernatants of H5200VVASH2 compared to that in H520CTRL. The level of VASH2 protein in different supernatants was detected by ELISA. B We first compared the proliferation of H5200V-VASH2 by CCK-8 assay. C The apoptotic in H520CTRL and H5200V-VASH2 cells was measured using Annexin-V-FITC. D The migration and invasion potentials were compared by Wound Healing Assay. Images were taken at 0, 24h and 48h later to measure the distance of the wound. E The Matrigel and Trans-well were used to carry out lung cancer cell invasion assays to build invading chambers for separating high invasive and low invasive cells. The stained membranes were photographed through the microscope and invading cells were counted after 48h. F, G, H, I HUVECs and HLECs were co-cultured with the supernatants from either H5200V-VASH2 orH520CTRL cells. The HUVECs and HLECs cultured in DMEM medium were used as negative controls. The numbers of forming tubes in each group were assessed. 
$\mathrm{F}$
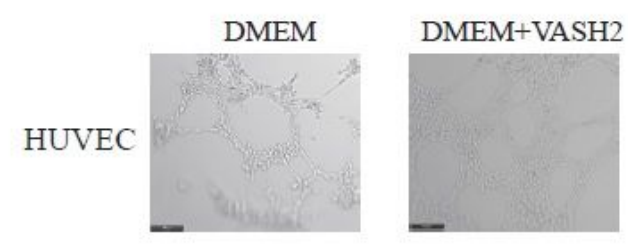

DMEM+VASH2+VASH2 AB

G
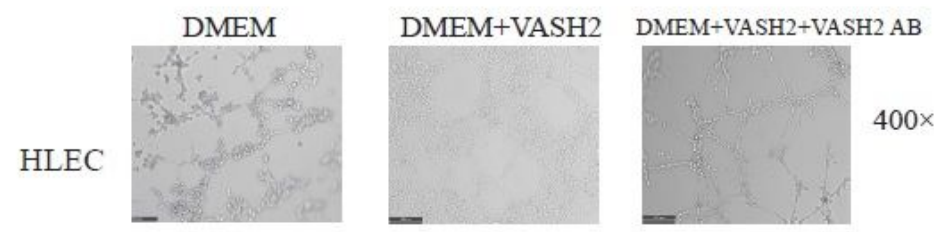

$\mathrm{H}$

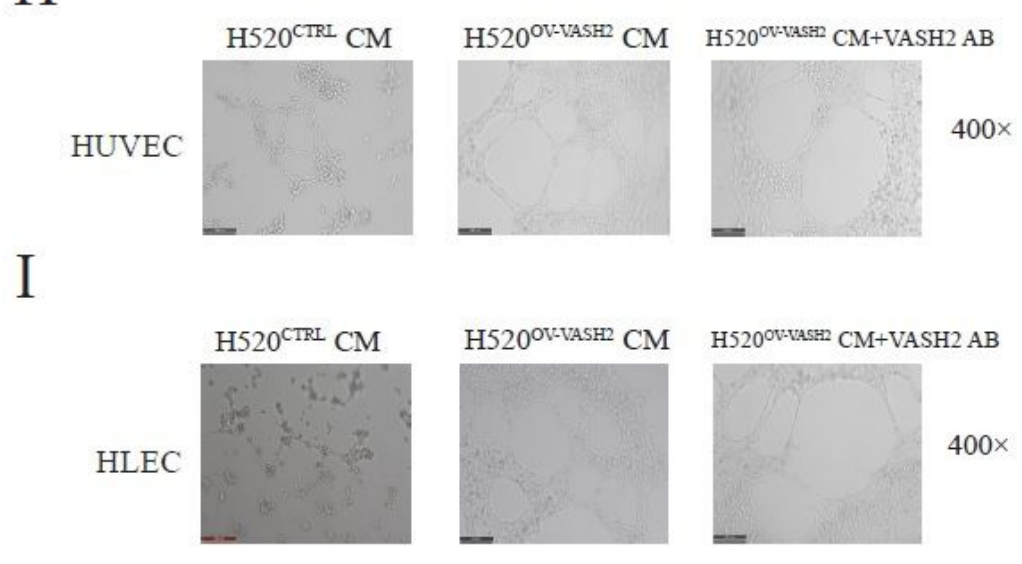

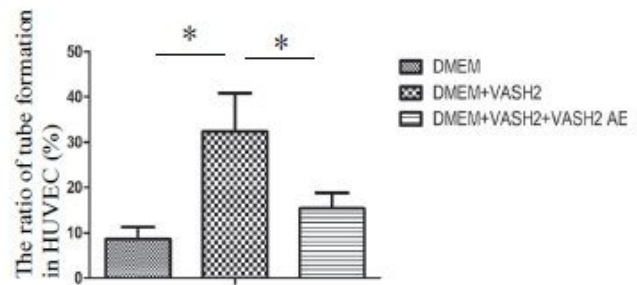
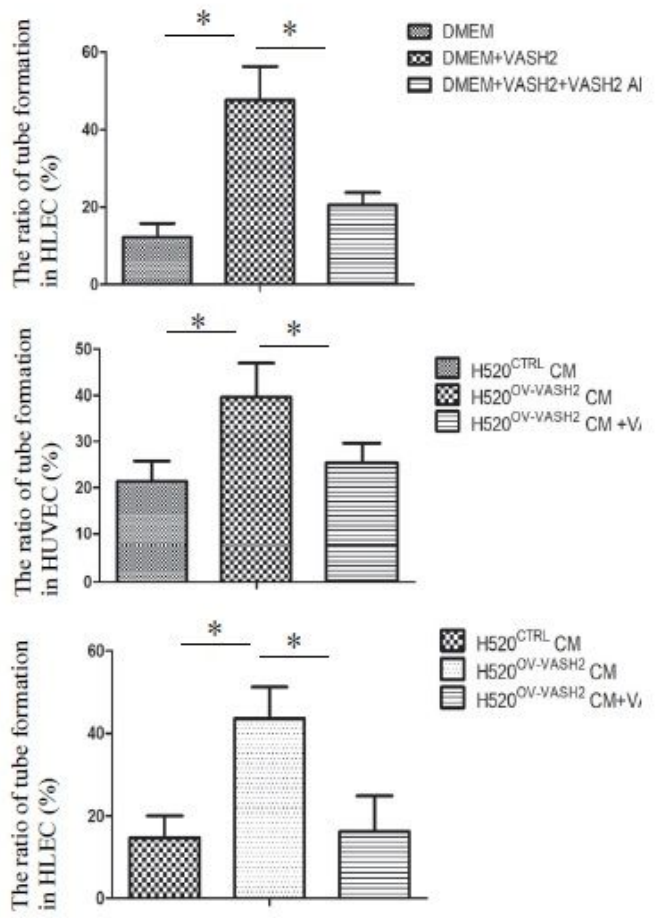

\section{Figure 2}

VASH2 accelerated the proliferation and invasion potential of LUSC cells, and promoted tube-formation capacity of HUVEC and HLEC cells A High level of VASH2 was confirmed in the supernatants of H5200VVASH2 compared to that in H520CTRL. The level of VASH2 protein in different supernatants was detected by ELISA. B We first compared the proliferation of H5200V-VASH2 by CCK-8 assay. C The apoptotic in H520CTRL and H5200V-VASH2 cells was measured using Annexin-V-FITC. D The migration and invasion potentials were compared by Wound Healing Assay. Images were taken at 0, 24h and 48h later to measure the distance of the wound. E The Matrigel and Trans-well were used to carry out lung cancer cell invasion assays to build invading chambers for separating high invasive and low invasive cells. The stained membranes were photographed through the microscope and invading cells were counted after 48h. F, G, H, I HUVECs and HLECs were co-cultured with the supernatants from either H5200V-VASH2 orH520CTRL cells. The HUVECs and HLECs cultured in DMEM medium were used as negative controls. The numbers of forming tubes in each group were assessed. 
$\mathrm{F}$
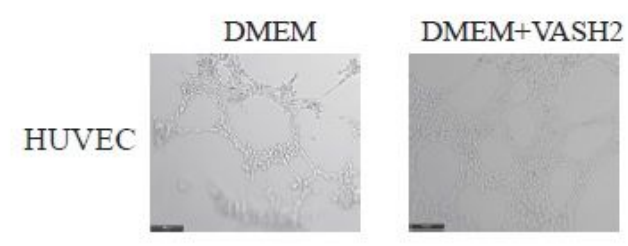

DMEM+VASH2+VASH2 AB

G
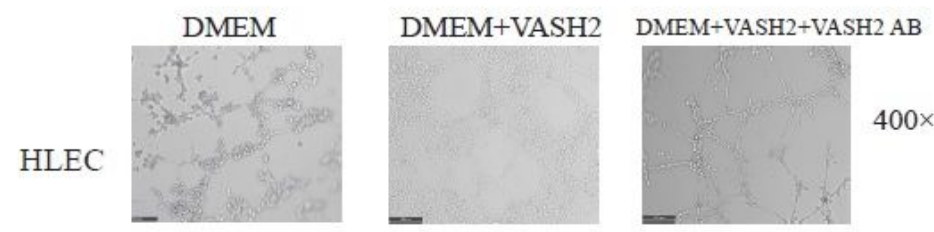

$\mathrm{H}$

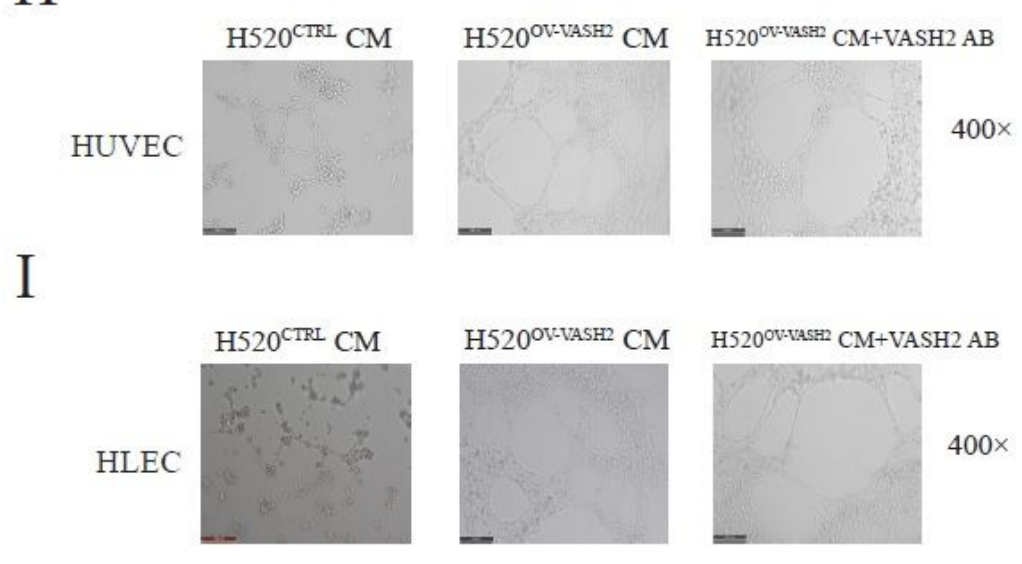

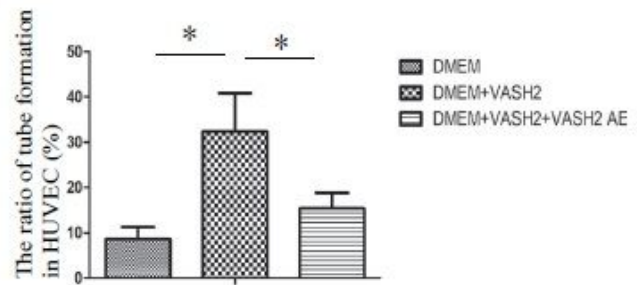
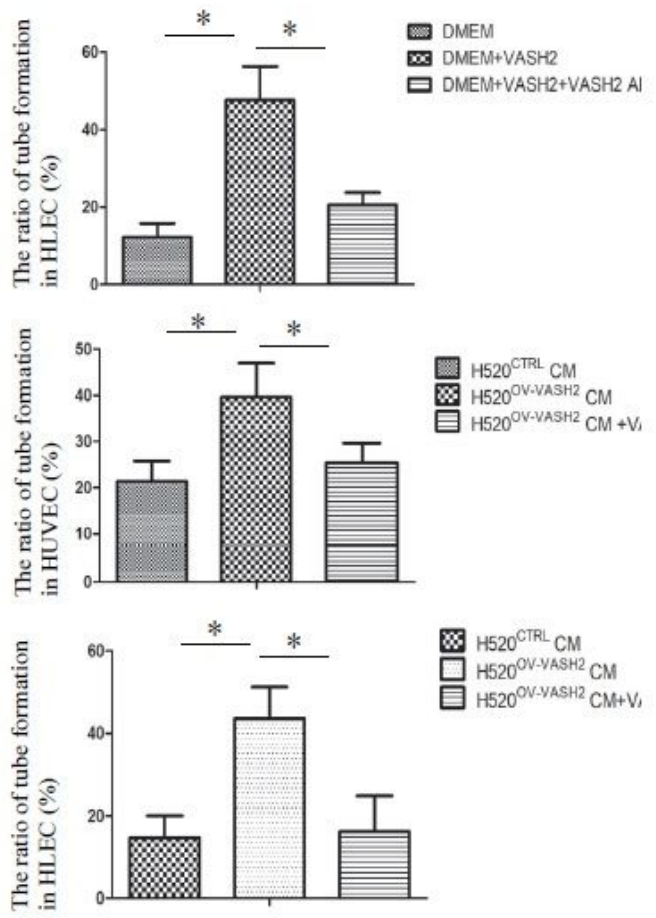

\section{Figure 2}

VASH2 accelerated the proliferation and invasion potential of LUSC cells, and promoted tube-formation capacity of HUVEC and HLEC cells A High level of VASH2 was confirmed in the supernatants of H5200VVASH2 compared to that in H520CTRL. The level of VASH2 protein in different supernatants was detected by ELISA. B We first compared the proliferation of H5200V-VASH2 by CCK-8 assay. C The apoptotic in H520CTRL and H5200V-VASH2 cells was measured using Annexin-V-FITC. D The migration and invasion potentials were compared by Wound Healing Assay. Images were taken at 0, 24h and 48h later to measure the distance of the wound. E The Matrigel and Trans-well were used to carry out lung cancer cell invasion assays to build invading chambers for separating high invasive and low invasive cells. The stained membranes were photographed through the microscope and invading cells were counted after 48h. F, G, H, I HUVECs and HLECs were co-cultured with the supernatants from either H5200V-VASH2 orH520CTRL cells. The HUVECs and HLECs cultured in DMEM medium were used as negative controls. The numbers of forming tubes in each group were assessed. 
A

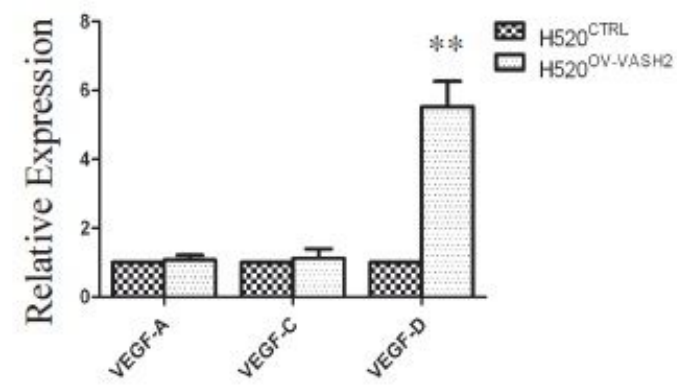

$\mathrm{C}$

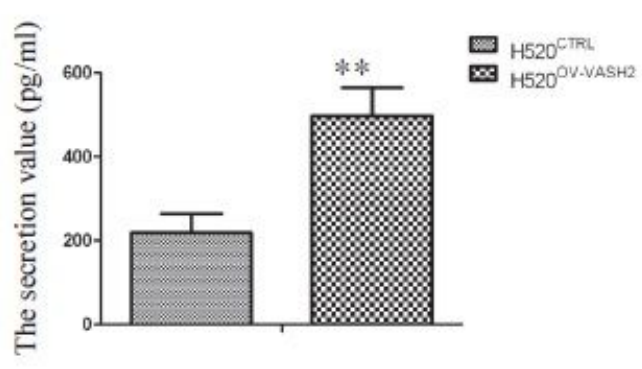

$\mathrm{E}$

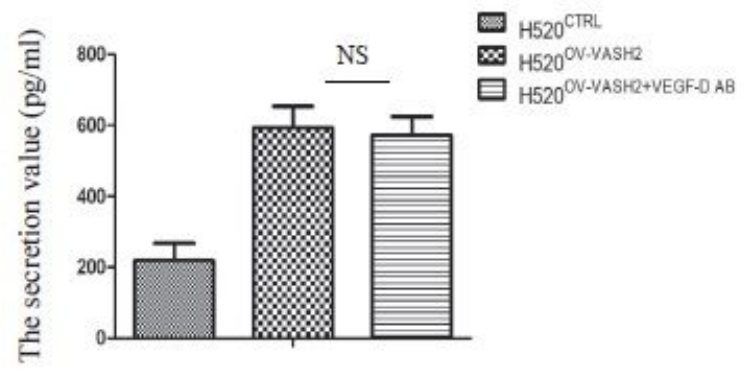

$\mathrm{B}$
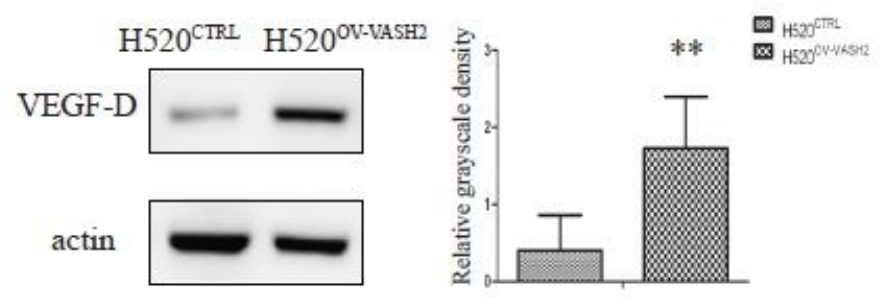

$\mathrm{D}$

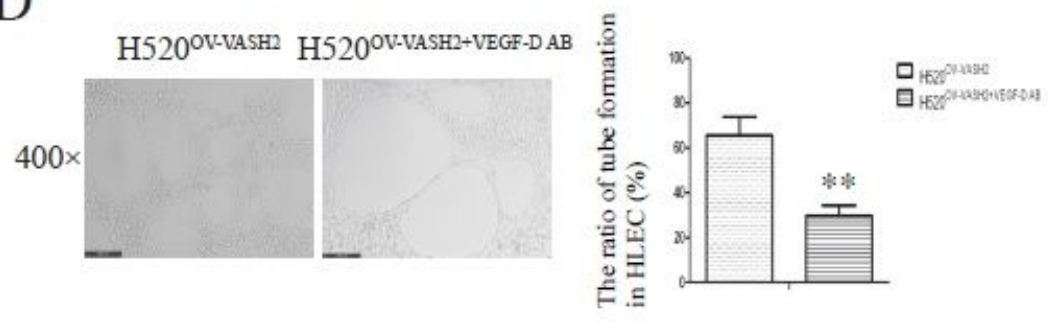

F

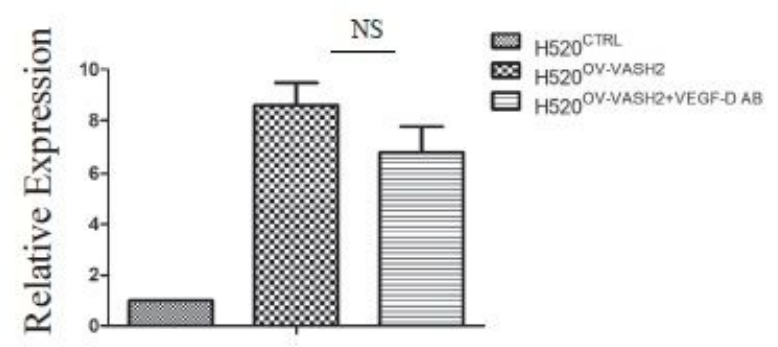

\section{Figure 3}

VASH2 facilitated lymphangiogenesis via up-regulating VEGF-D production in LUSC cells A VEGF family members inH5200V-VASH2 cells, including VEGF-A, VEGF-Cand VEGF-D, were measured using qPCR assay. B The protein level of VEGF-D in H520OV-VASH2 cells was detected by Western blot analysis. C The level of VEGF-D protein in the supernatants of H5200V-VASH2 and H520CTRL was compared by ELISA assay. D Test the influence of specific VEGF-D blocking antibody on the tube formation of HLECs co-cultured with H5200V-VASH2. E, F VEGF-D antibody was added to the two groups of cells respectively. The effect of VEGF-D on VASH2 production and release was detected by ELISA and PCR. 
A

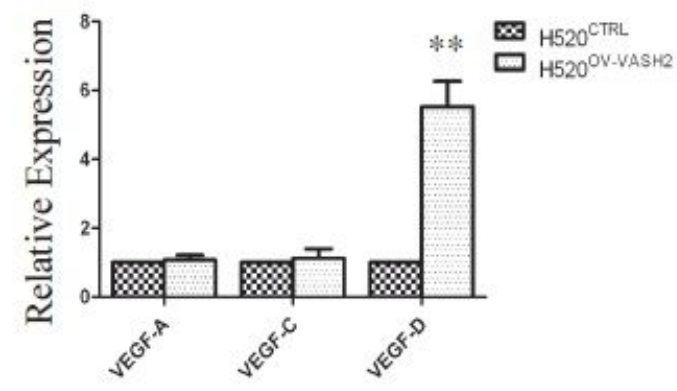

$\mathrm{C}$

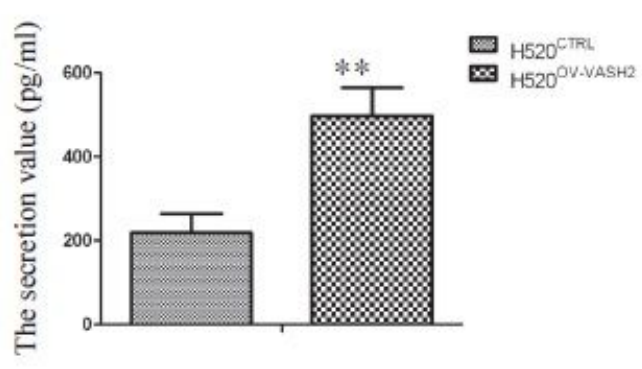

$\mathrm{E}$

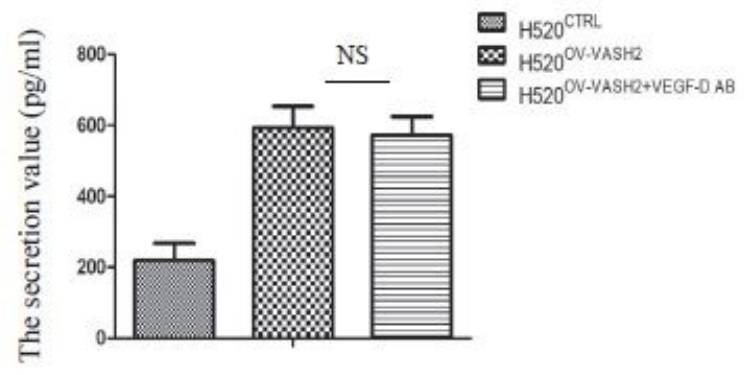

$\mathrm{B}$
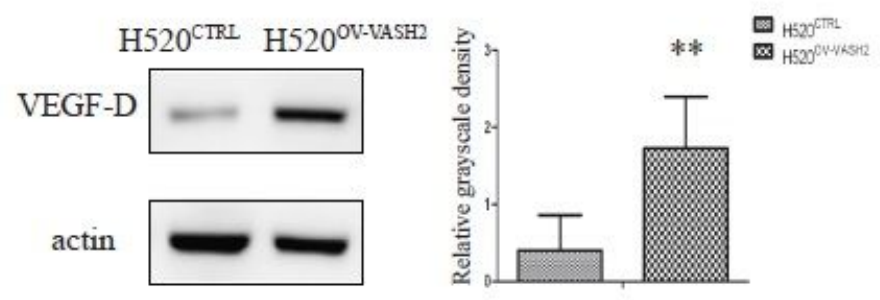

$\mathrm{D}$

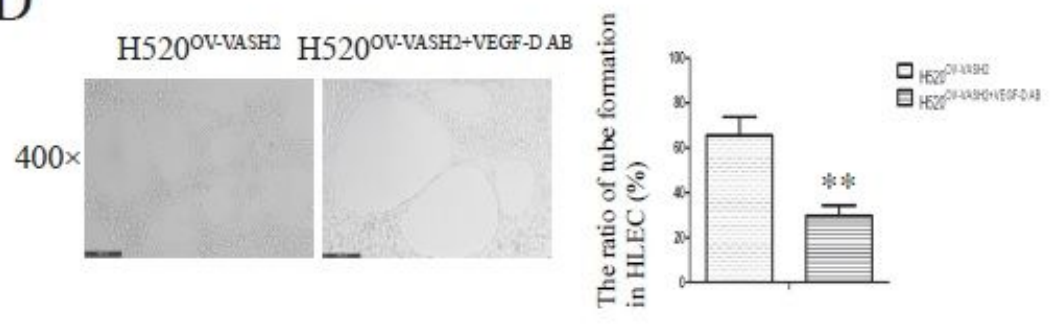

F

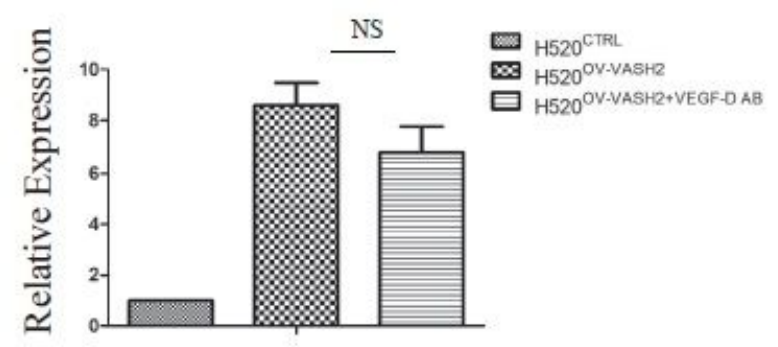

\section{Figure 3}

VASH2 facilitated lymphangiogenesis via up-regulating VEGF-D production in LUSC cells A VEGF family members inH5200V-VASH2 cells, including VEGF-A, VEGF-Cand VEGF-D, were measured using qPCR assay. B The protein level of VEGF-D in H520OV-VASH2 cells was detected by Western blot analysis. C The level of VEGF-D protein in the supernatants of H5200V-VASH2 and H520CTRL was compared by ELISA assay. D Test the influence of specific VEGF-D blocking antibody on the tube formation of HLECs co-cultured with H5200V-VASH2. E, F VEGF-D antibody was added to the two groups of cells respectively. The effect of VEGF-D on VASH2 production and release was detected by ELISA and PCR. 
A

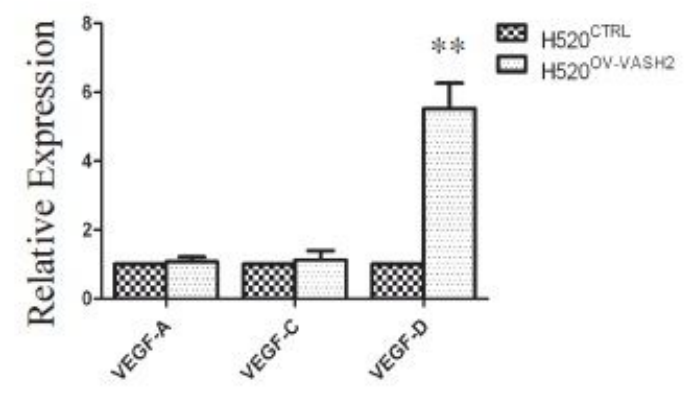

$\mathrm{C}$

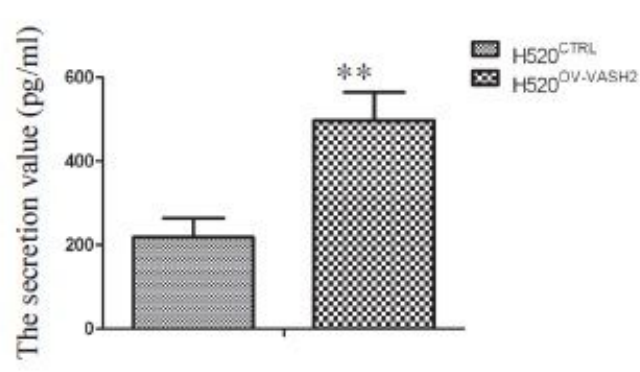

$\mathrm{E}$

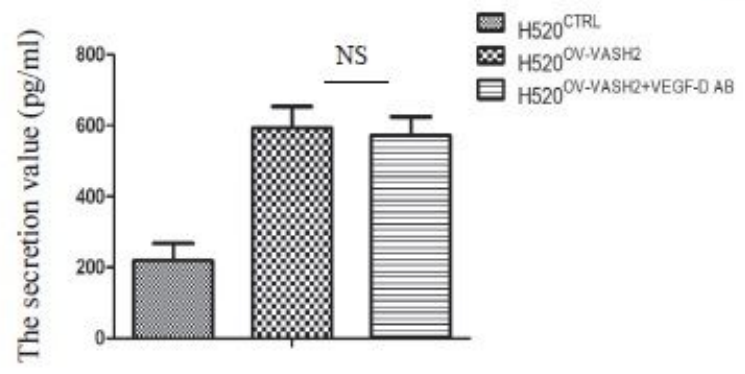

B

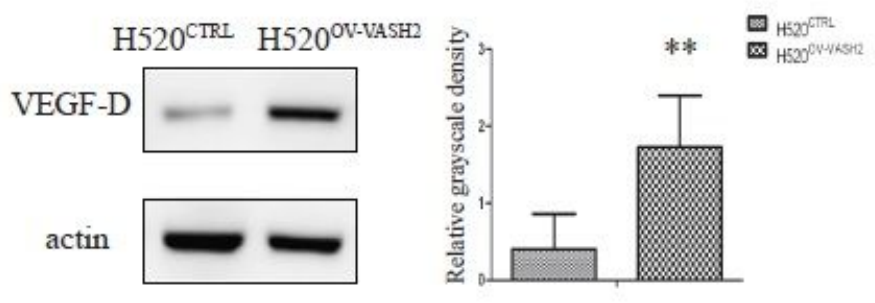

$\mathrm{D}$

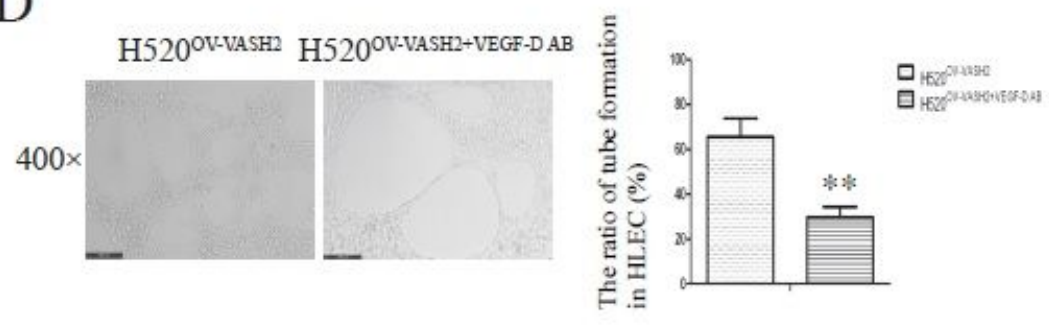

F

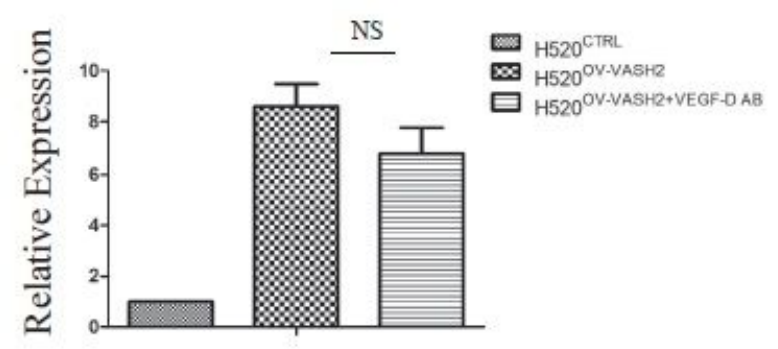

\section{Figure 3}

VASH2 facilitated lymphangiogenesis via up-regulating VEGF-D production in LUSC cells A VEGF family members inH5200V-VASH2 cells, including VEGF-A, VEGF-Cand VEGF-D, were measured using qPCR assay. B The protein level of VEGF-D in H520OV-VASH2 cells was detected by Western blot analysis. C The level of VEGF-D protein in the supernatants of H5200V-VASH2 and H520CTRL was compared by ELISA assay. D Test the influence of specific VEGF-D blocking antibody on the tube formation of HLECs co-cultured with H5200V-VASH2. E, F VEGF-D antibody was added to the two groups of cells respectively. The effect of VEGF-D on VASH2 production and release was detected by ELISA and PCR. 
A

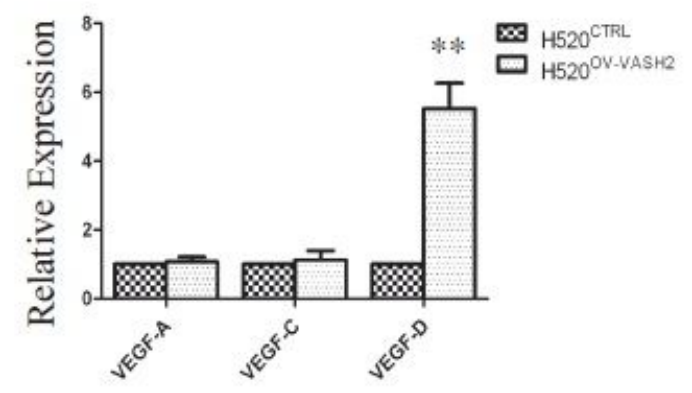

$\mathrm{C}$

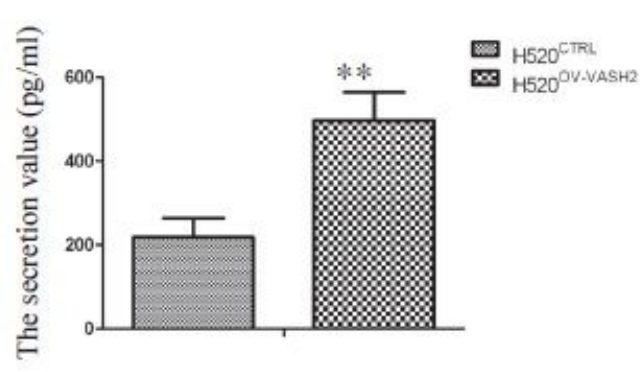

$\mathrm{E}$

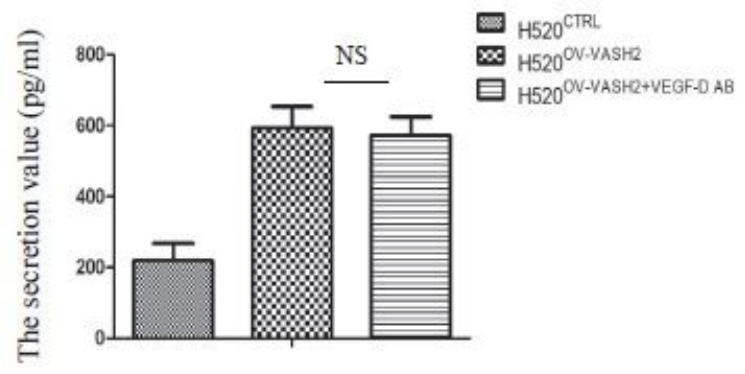

B

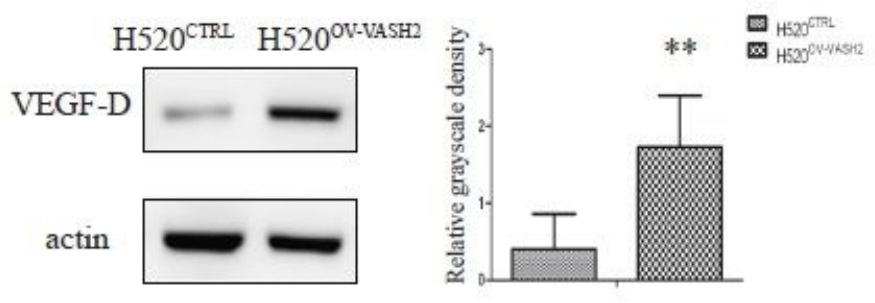

$\mathrm{D}$

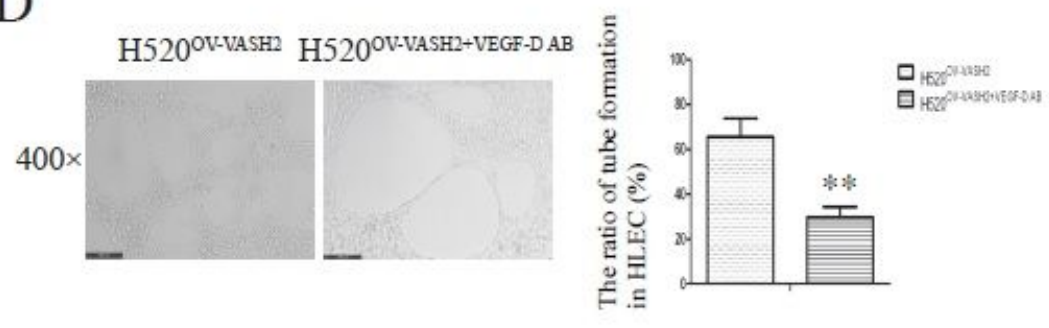

F

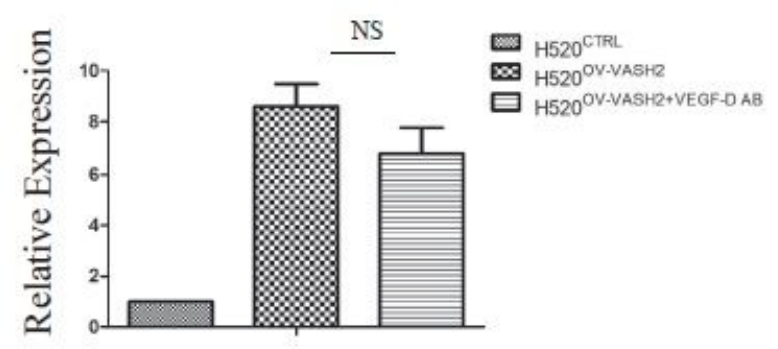

\section{Figure 3}

VASH2 facilitated lymphangiogenesis via up-regulating VEGF-D production in LUSC cells A VEGF family members inH5200V-VASH2 cells, including VEGF-A, VEGF-Cand VEGF-D, were measured using qPCR assay. B The protein level of VEGF-D in H520OV-VASH2 cells was detected by Western blot analysis. C The level of VEGF-D protein in the supernatants of H5200V-VASH2 and H520CTRL was compared by ELISA assay. D Test the influence of specific VEGF-D blocking antibody on the tube formation of HLECs co-cultured with H5200V-VASH2. E, F VEGF-D antibody was added to the two groups of cells respectively. The effect of VEGF-D on VASH2 production and release was detected by ELISA and PCR. 
A

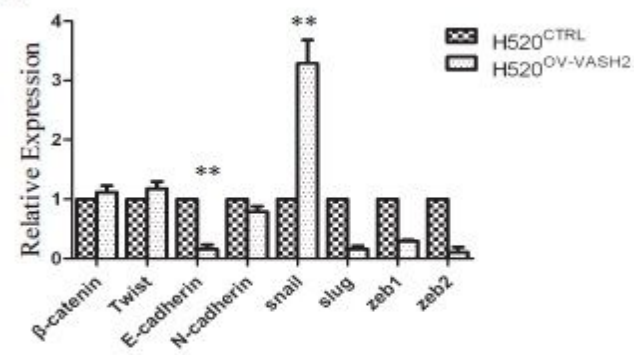

D

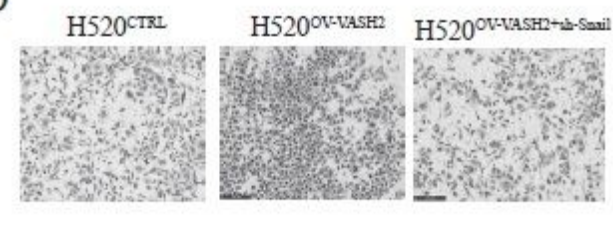

F

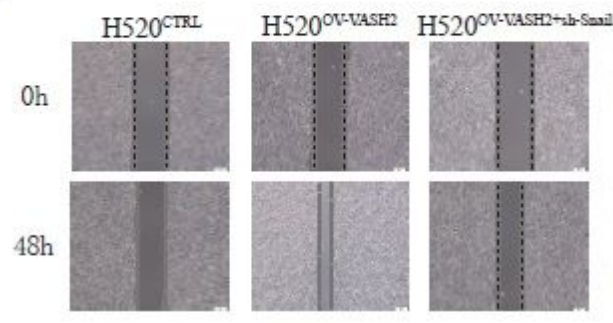

B

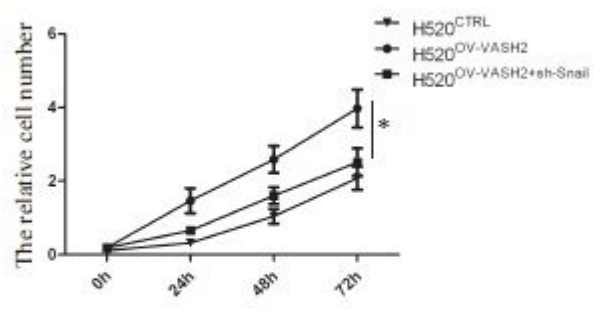

C

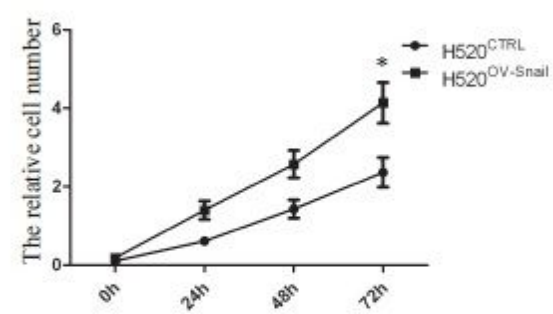

E
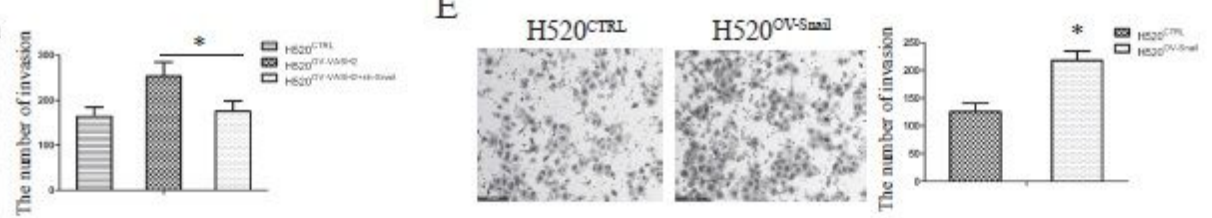

G

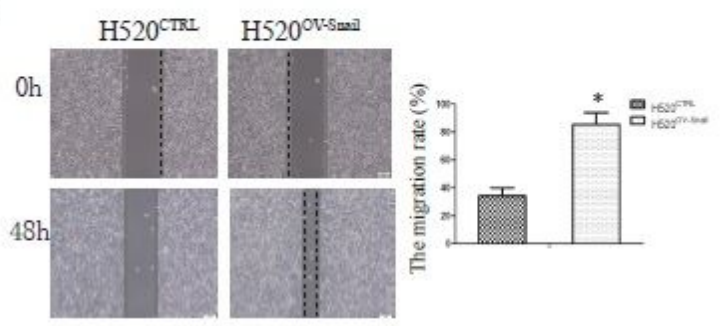

\section{Figure 4}

VASH2 enhanced tumor proliferation and invasion via up-regulating Snail expression in LUSC cells A The transcription of multiple EMT-related biomarkers were detected using qPCR assay. B, C We compared the proliferation of H520CTRL, H5200V-VASH2, H5200V-VASH2+sh-Snail and H520OV-Snail cells using the CCK-8 staining assay. D, E The cell invasion were compared trans-well assays. F, G The cell migration were compared using the wound healing. 
A

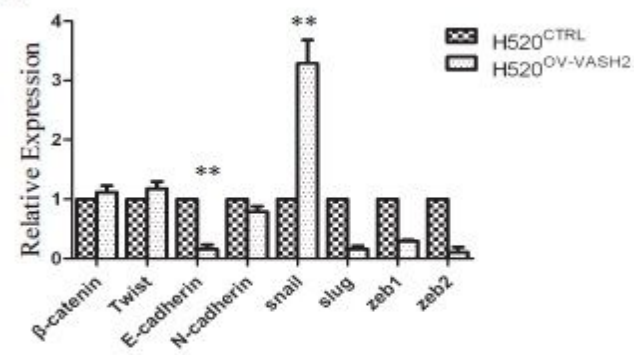

D

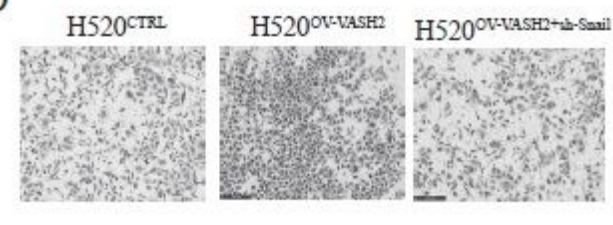

F

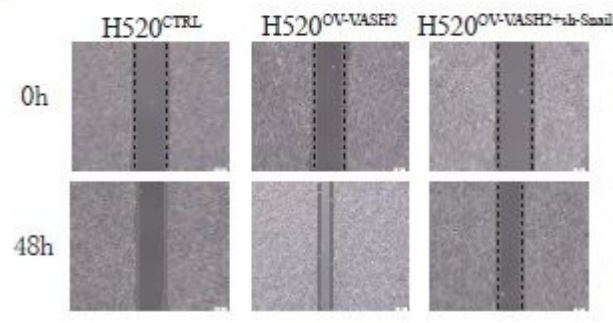

B

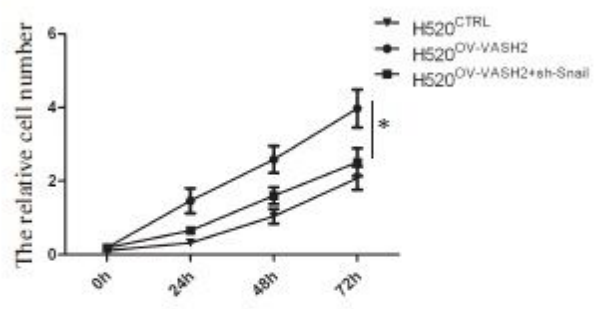

C

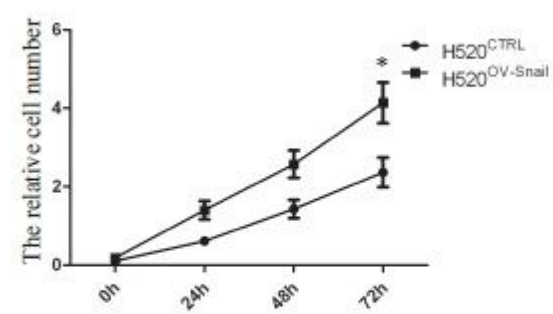

E
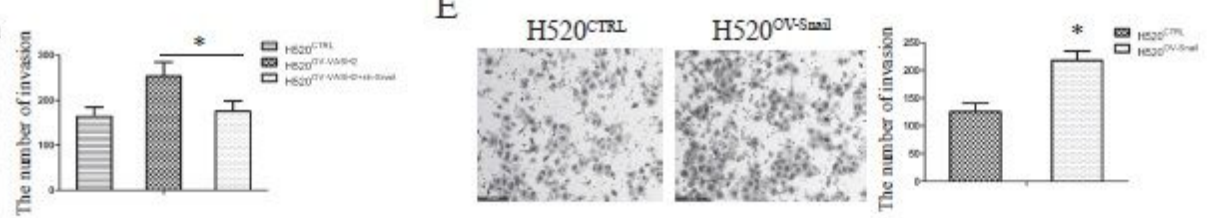

G

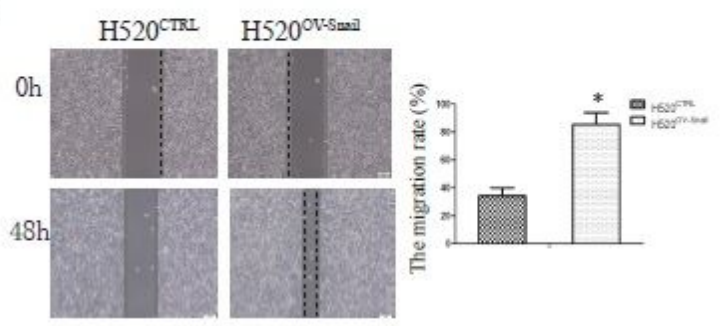

\section{Figure 4}

VASH2 enhanced tumor proliferation and invasion via up-regulating Snail expression in LUSC cells A The transcription of multiple EMT-related biomarkers were detected using qPCR assay. B, C We compared the proliferation of H520CTRL, H5200V-VASH2, H5200V-VASH2+sh-Snail and H520OV-Snail cells using the CCK-8 staining assay. D, E The cell invasion were compared trans-well assays. F, G The cell migration were compared using the wound healing. 
A

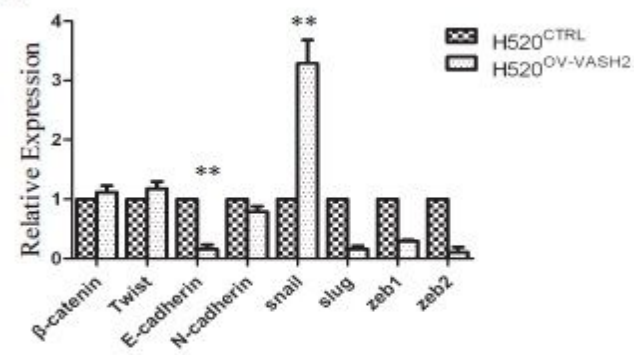

D

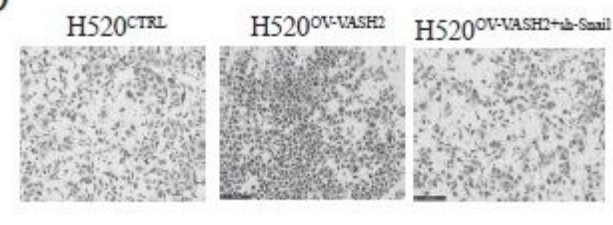

F

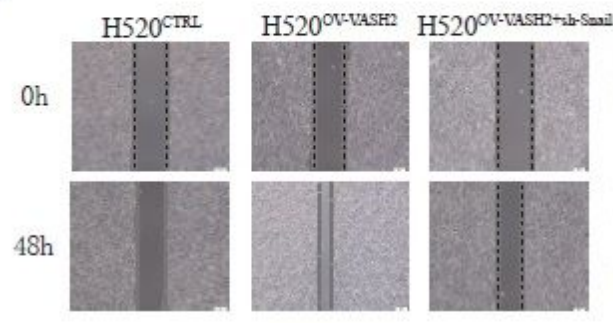

B

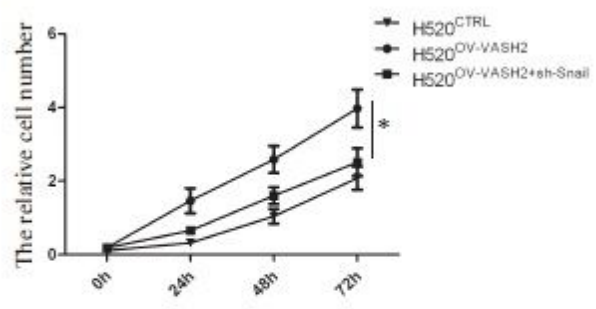

C

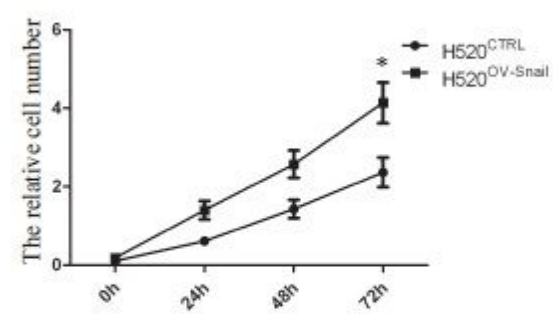

E
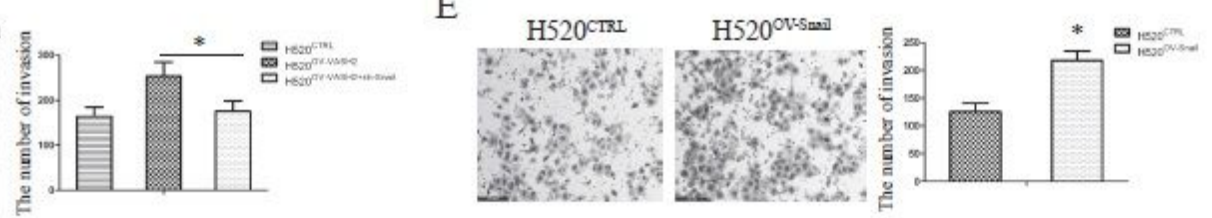

G

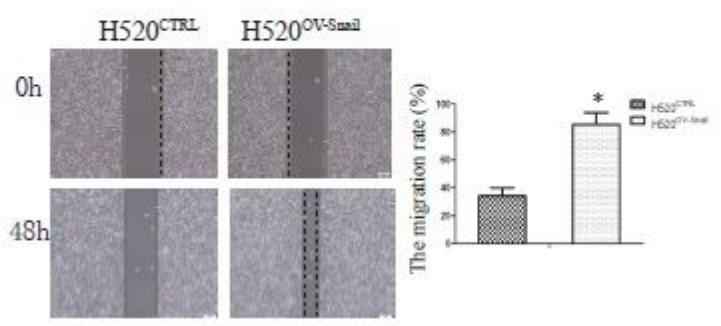

\section{Figure 4}

VASH2 enhanced tumor proliferation and invasion via up-regulating Snail expression in LUSC cells A The transcription of multiple EMT-related biomarkers were detected using qPCR assay. B, C We compared the proliferation of H520CTRL, H5200V-VASH2, H5200V-VASH2+sh-Snail and H520OV-Snail cells using the CCK-8 staining assay. D, E The cell invasion were compared trans-well assays. F, G The cell migration were compared using the wound healing. 


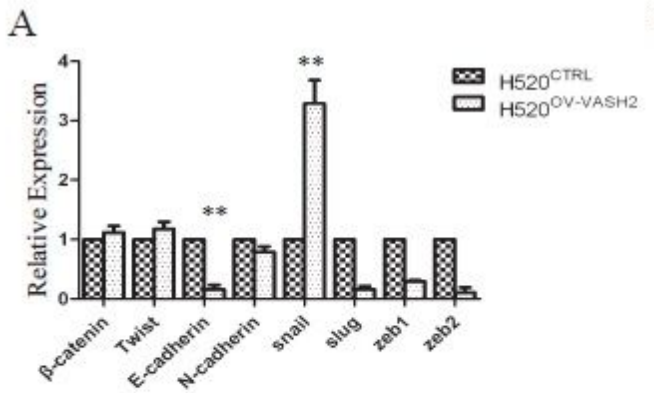

D

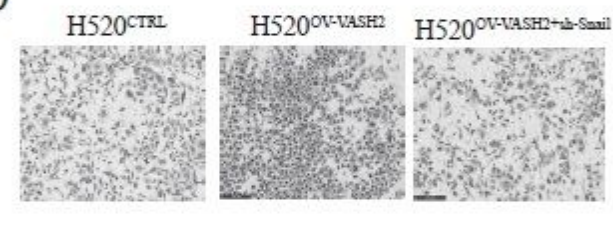

F

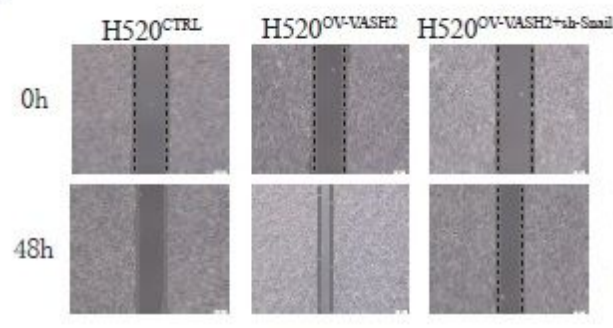

B

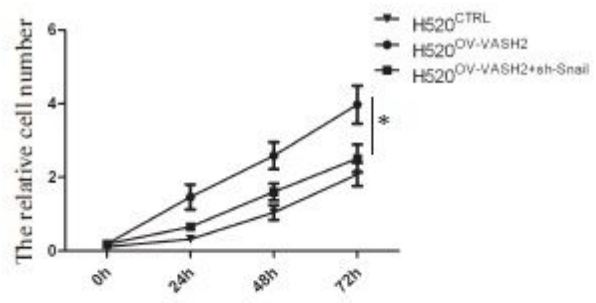

$\mathrm{C}$

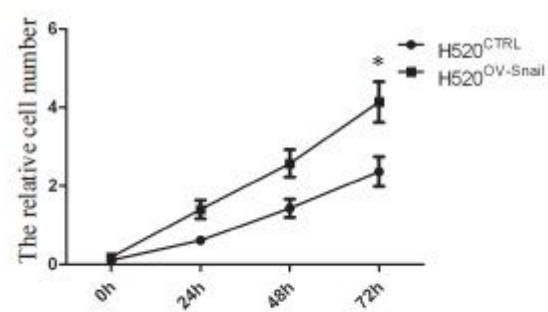

E

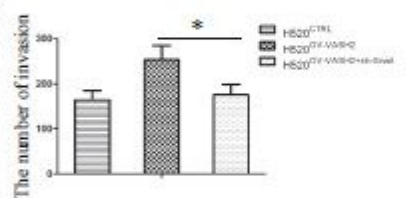

E

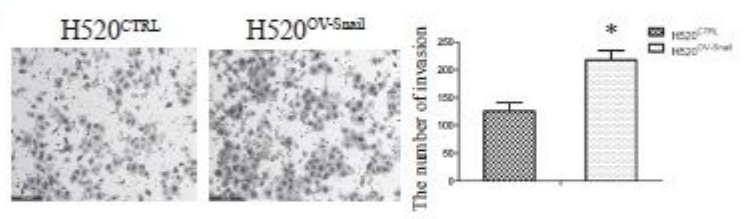

G

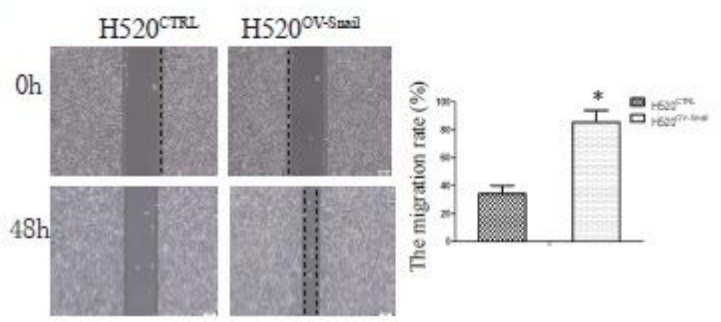

\section{Figure 4}

VASH2 enhanced tumor proliferation and invasion via up-regulating Snail expression in LUSC cells A The transcription of multiple EMT-related biomarkers were detected using QPCR assay. B, C We compared the proliferation of H520CTRL, H520OV-VASH2, H520OV-VASH2+sh-Snail and H520OV-Snail cells using the CCK-8 staining assay. D, E The cell invasion were compared trans-well assays. F, G The cell migration were compared using the wound healing. 
A
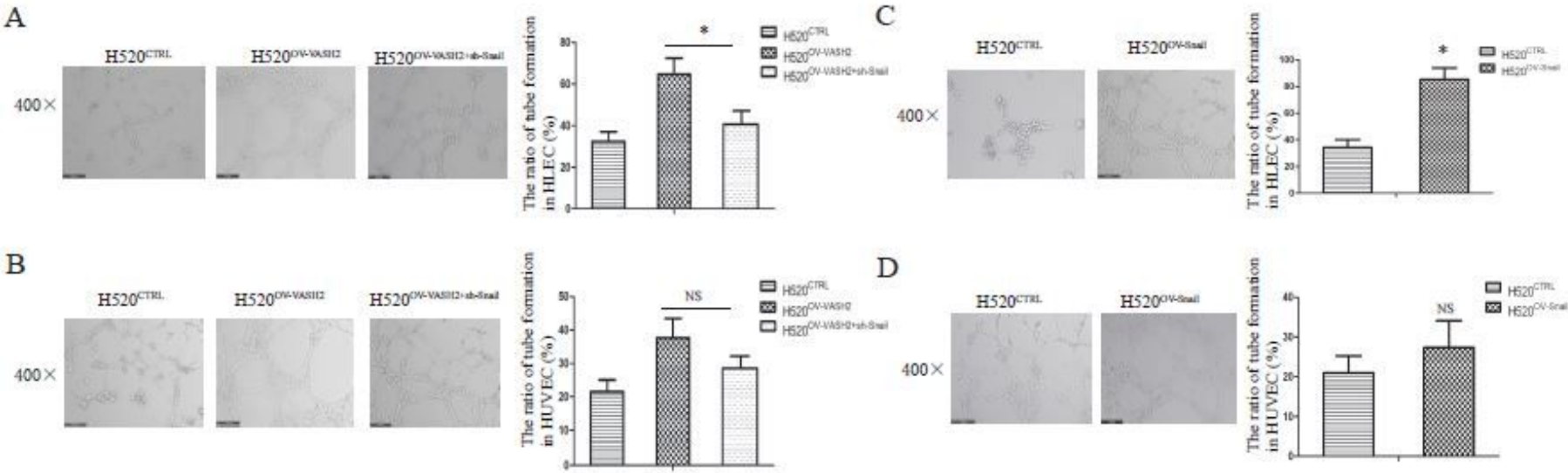

$\mathrm{E}$

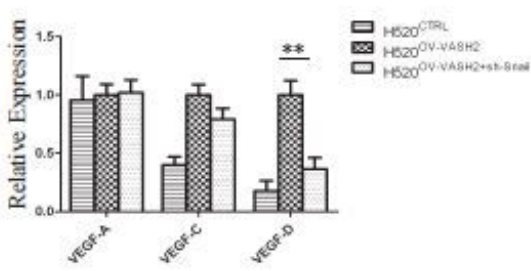

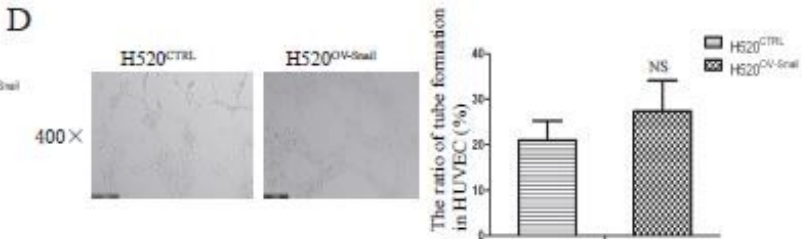

$\mathrm{F}$

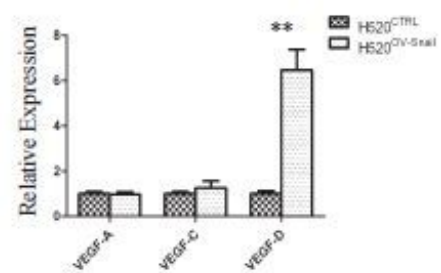

\section{Figure 5}

Snail regulated VASH2-related lymphangiogenesis via stimulating VEGF-D productionin LUSC cell A, B, C, D We transfected H5200V-VASH2 cells with sh-Snail recombinant lentivirus and compared the tube formation capacity of HLECs or HUVECs co-cultured with either H520CTRL, H520OV-VASH2, H520OVVASH2+sh-Snail or H5200V-Snail cells. E, F In order to determine if Snail regulated VASH2-stimulated VEGF-D production, H520CTRL, H5200V-VASH2, H5200V-VASH2+sh-Snail and H5200V-Snail cells were collected to compare the mRNA levels of VEGF-A, VEGF-C and VEGF-D at by qPCR assay.

A
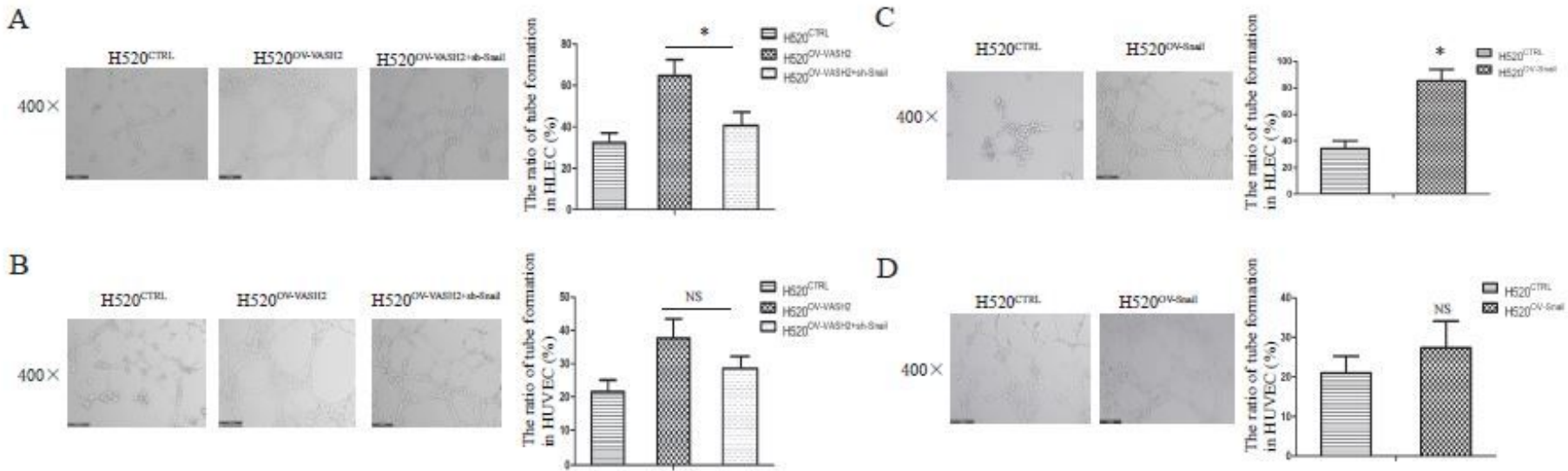

E

F
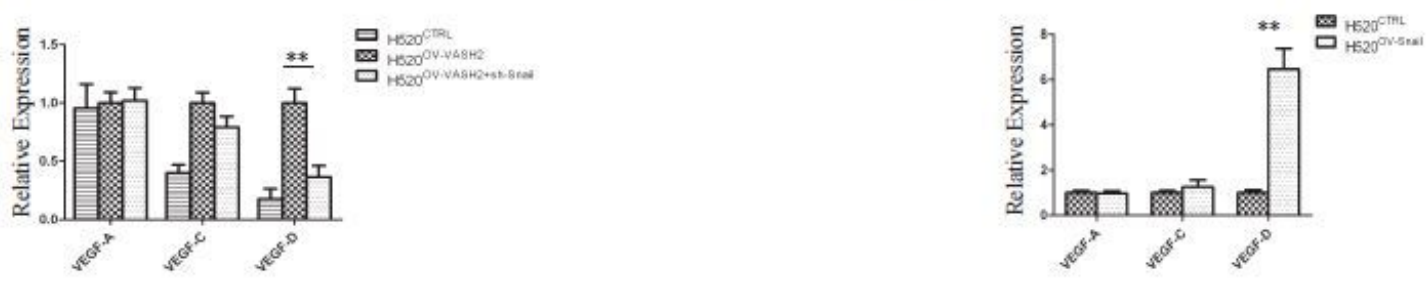


\section{Figure 5}

Snail regulated VASH2-related lymphangiogenesis via stimulating VEGF-D productionin LUSC cell A, B, C, D We transfected H5200V-VASH2 cells with sh-Snail recombinant lentivirus and compared the tube formation capacity of HLECs or HUVECs co-cultured with either H520CTRL, H520OV-VASH2, H520OVVASH2+sh-Snail or H5200V-Snail cells. E, F In order to determine if Snail regulated VASH2-stimulated VEGF-D production, H520CTRL, H5200V-VASH2, H5200V-VASH2+sh-Snail and H5200V-Snail cells were collected to compare the mRNA levels of VEGF-A, VEGF-C and VEGF-D at by qPCR assay.

A
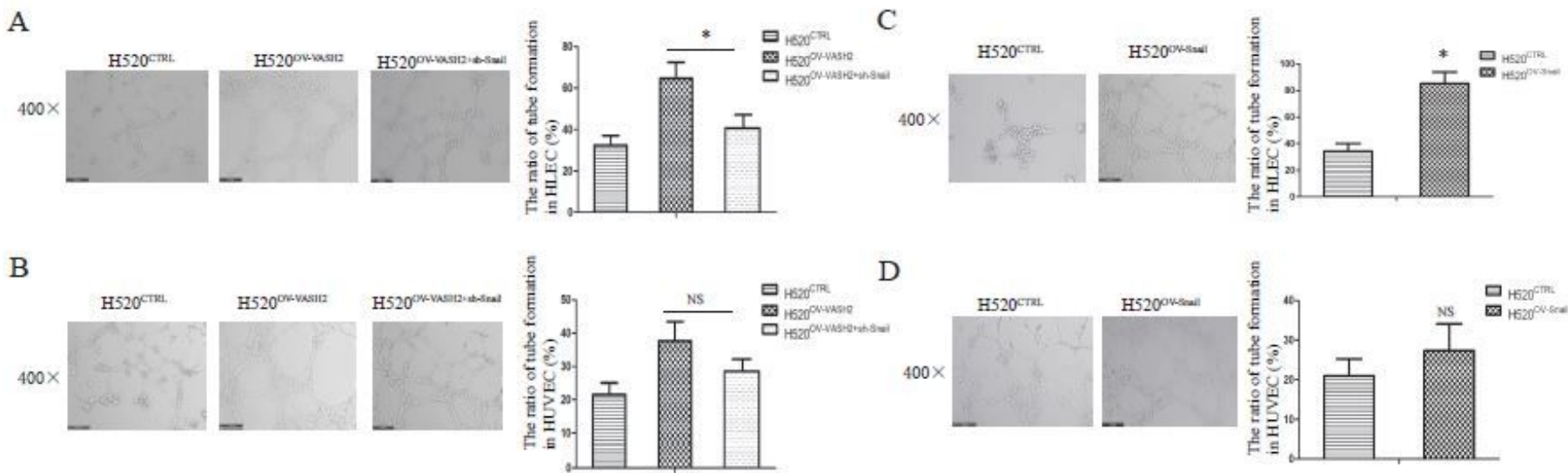

$\mathrm{E}$
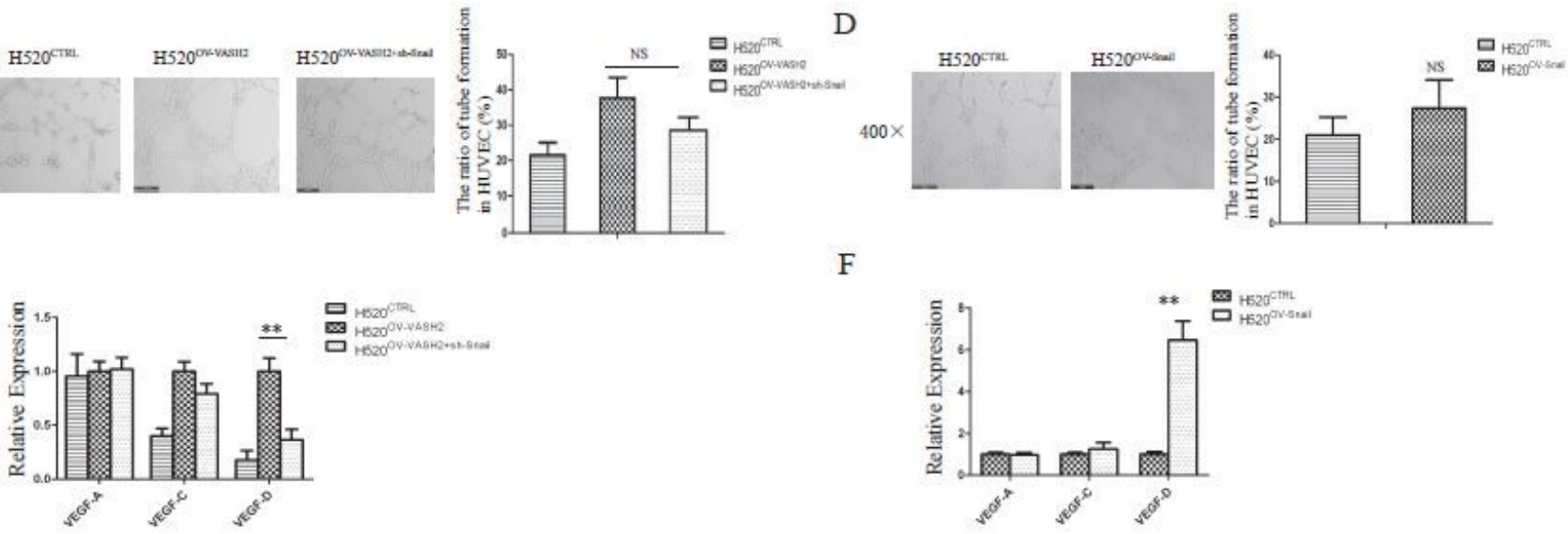

$\mathrm{F}$

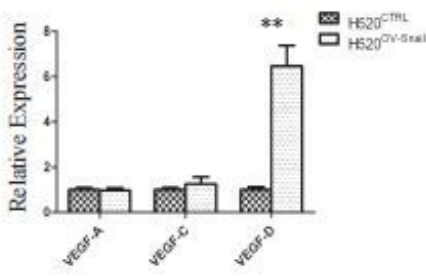

\section{Figure 5}

Snail regulated VASH2-related lymphangiogenesis via stimulating VEGF-D productionin LUSC cell A, B, C, D We transfected H5200V-VASH2 cells with sh-Snail recombinant lentivirus and compared the tube formation capacity of HLECs or HUVECs co-cultured with either H520CTRL, H520OV-VASH2, H520OVVASH2+sh-Snail or H5200V-Snail cells. E, F In order to determine if Snail regulated VASH2-stimulated VEGF-D production, H520CTRL, H5200V-VASH2, H5200V-VASH2+sh-Snail and H520OV-Snail cells were collected to compare the mRNA levels of VEGF-A, VEGF-C and VEGF-D at by qPCR assay. 
A
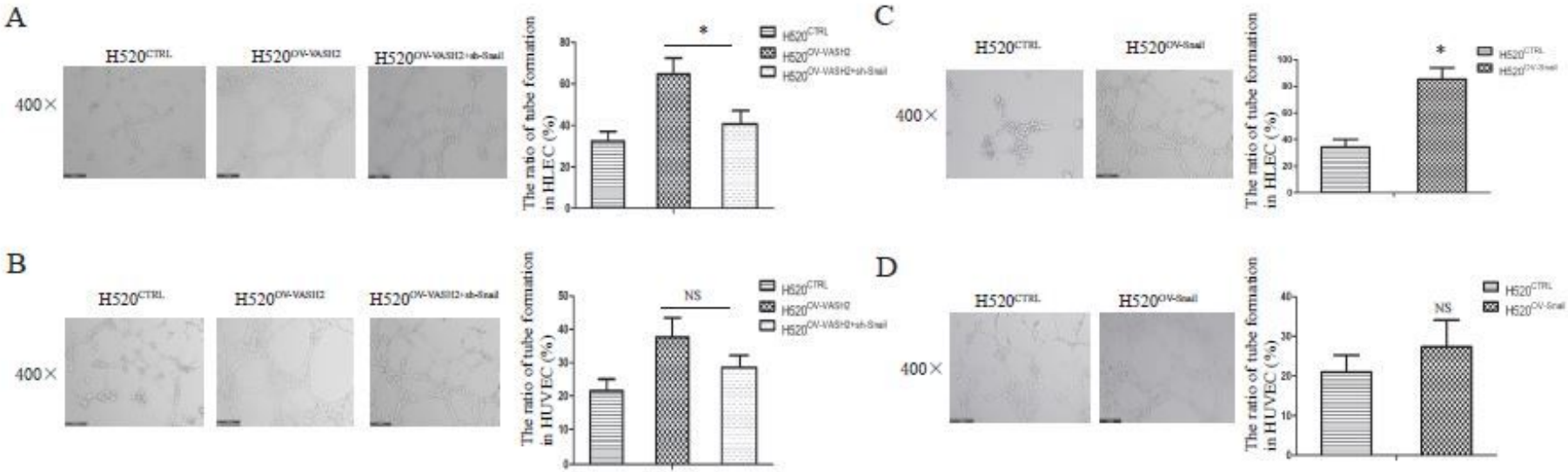

$\mathrm{E}$

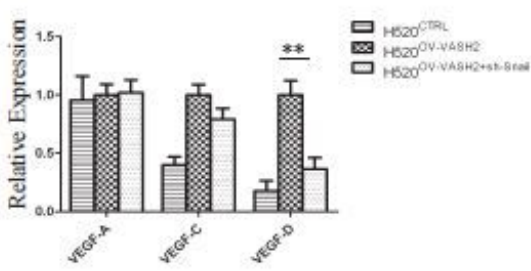

F

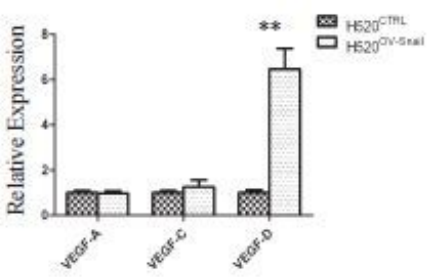

\section{Figure 5}

Snail regulated VASH2-related lymphangiogenesis via stimulating VEGF-D productionin LUSC cell A, B, C, D We transfected H5200V-VASH2 cells with sh-Snail recombinant lentivirus and compared the tube formation capacity of HLECs or HUVECs co-cultured with either H520CTRL, H520OV-VASH2, H520OVVASH2+sh-Snail or H5200V-Snail cells. E, F In order to determine if Snail regulated VASH2-stimulated VEGF-D production, H520CTRL, H5200V-VASH2, H5200V-VASH2+sh-Snail and H5200V-Snail cells were collected to compare the mRNA levels of VEGF-A, VEGF-C and VEGF-D at by qPCR assay.

A

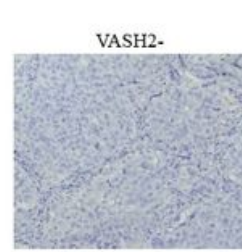

Snail-

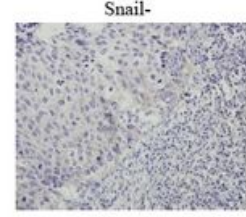

VEGF-D-

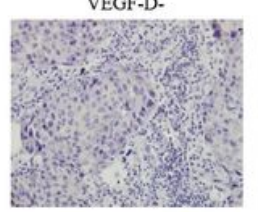

B

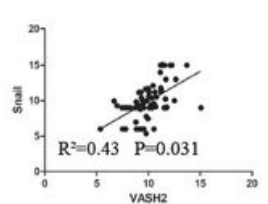

Snail +

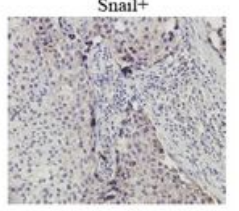

VEGF-D+

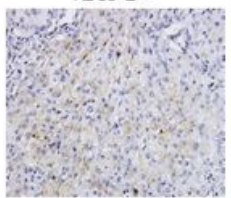

C

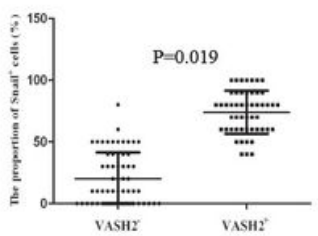

D
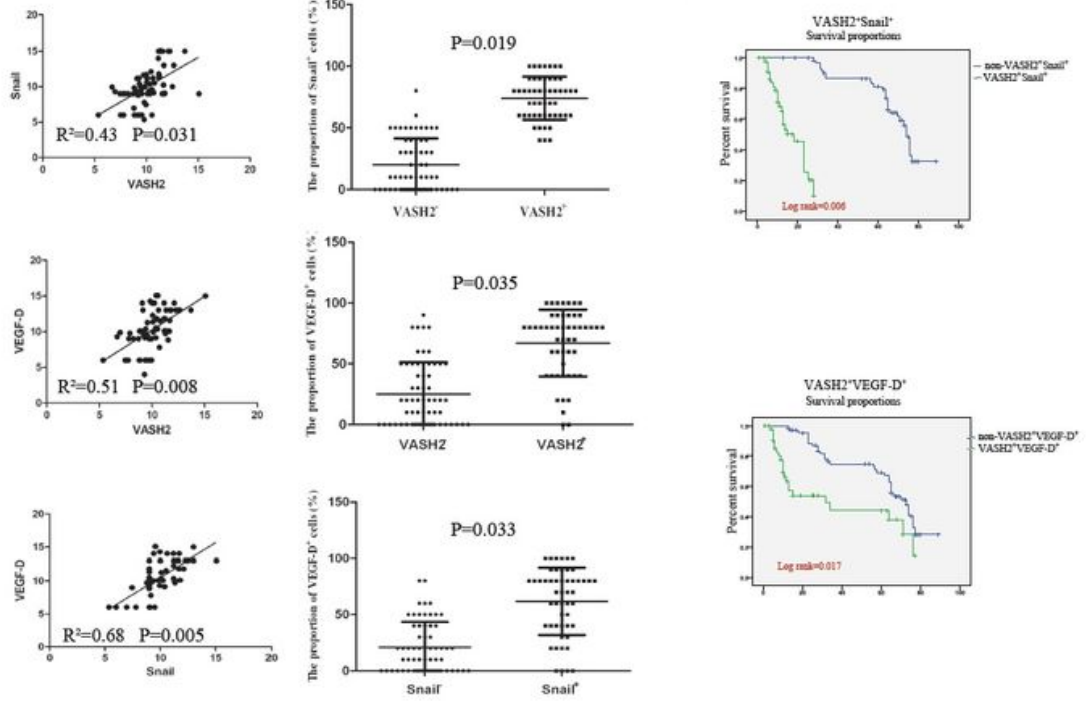


\section{Figure 6}

VASH2 was significantly correlated with either Snail orVEGF-D in primary LUSC tissues A The protein levels of Snail and VEGF-D in 64 cases of primary LUSC tissues were assessed using IHC staining assay. B We used qPCR to detect the mRNA levels of VASH2, Snail and VEGF-D in LUSC. C We analyzed the correlation of VASH2, Snail and VEGF-D by IHC results. D We divided them into double positive group. We compared the OS between VASH2+Snail+ LUSC patients and non-VASH2+Snail+ patients, as well as between VASH2+VEGF-D+ patients and non-VASH2+VEGF-D+ patients.

A
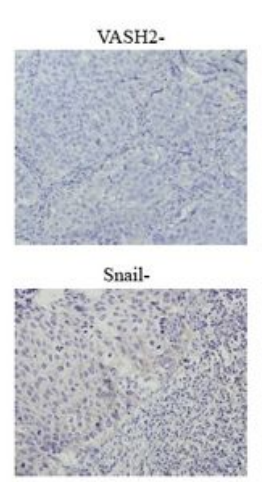

VEGF-D.

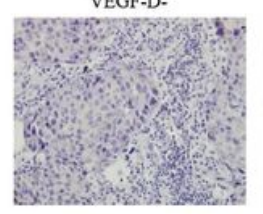

B

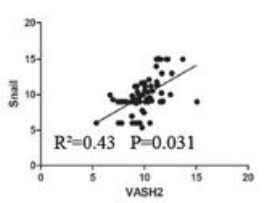

Snail+

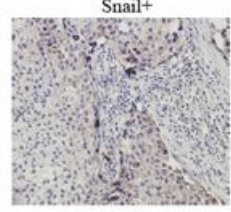

VEGF-D+

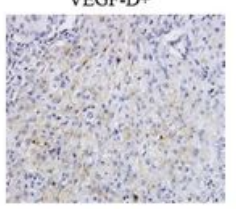

C
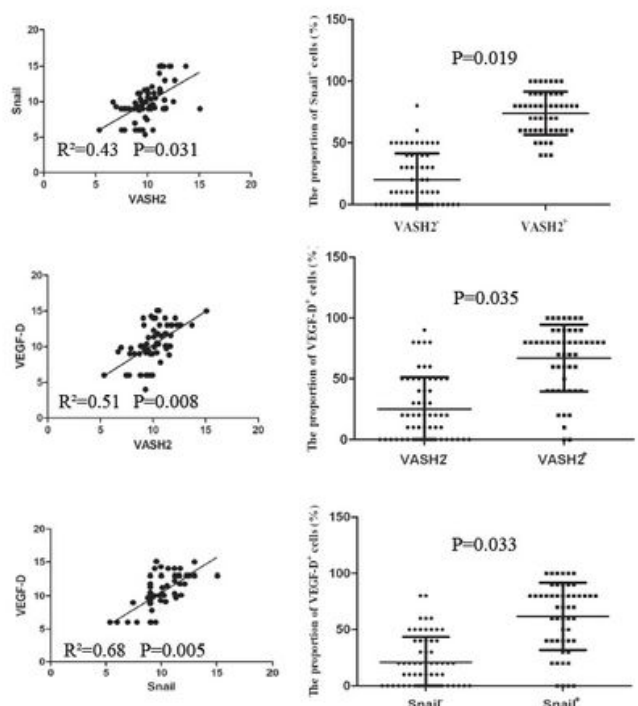

D
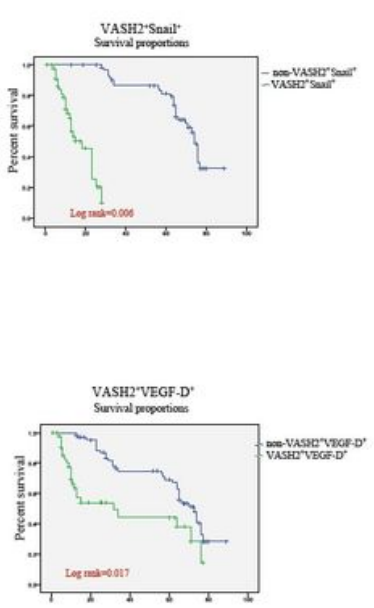

\section{Figure 6}

VASH2 was significantly correlated with either Snail orVEGF-D in primary LUSC tissues A The protein levels of Snail and VEGF-D in 64 cases of primary LUSC tissues were assessed using IHC staining assay. B We used qPCR to detect the mRNA levels of VASH2, Snail and VEGF-D in LUSC. C We analyzed the correlation of VASH2, Snail and VEGF-D by IHC results. D We divided them into double positive group. We compared the OS between VASH2+Snail+ LUSC patients and non-VASH2+Snail+ patients, as well as between VASH2+VEGF-D+ patients and non-VASH2+VEGF-D+ patients. 
A

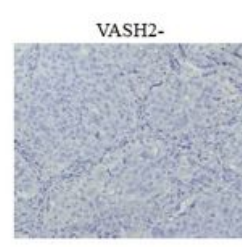

Snail-

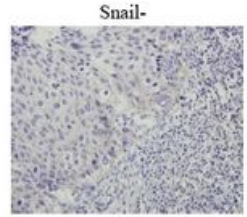

VEGF-D-

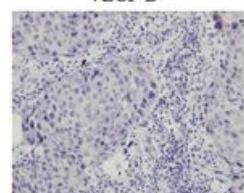

VASH2+

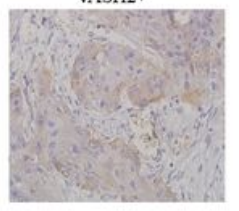

Snail +

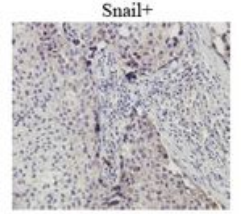

VEGF-D+

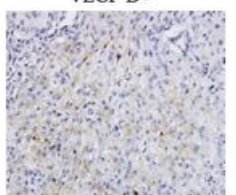

B
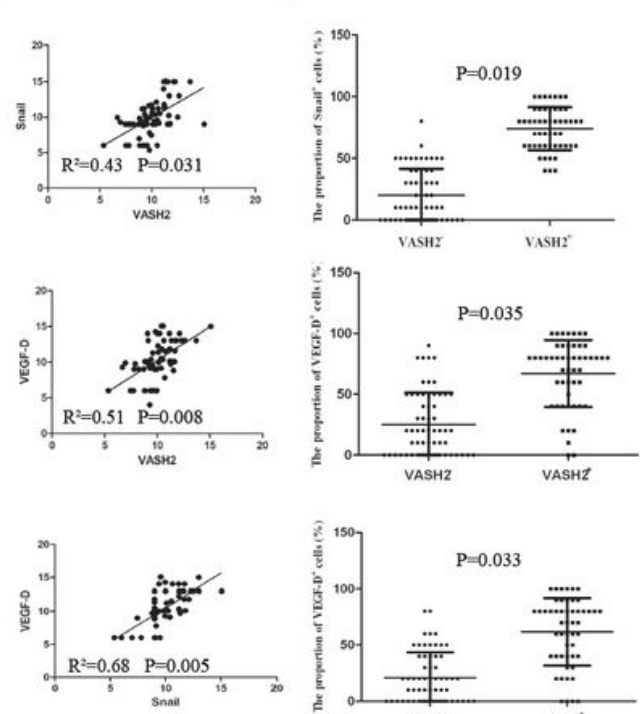

$\mathrm{D}$
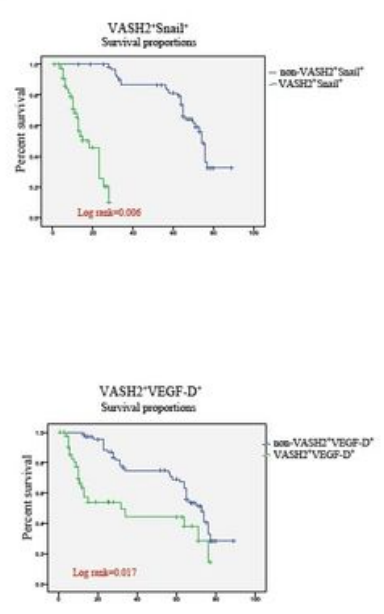

\section{Figure 6}

VASH2 was significantly correlated with either Snail orVEGF-D in primary LUSC tissues A The protein levels of Snail and VEGF-D in 64 cases of primary LUSC tissues were assessed using IHC staining assay. $B$ We used qPCR to detect the mRNA levels of VASH2, Snail and VEGF-D in LUSC. C We analyzed the correlation of VASH2, Snail and VEGF-D by IHC results. D We divided them into double positive group. We compared the OS between VASH2+Snail+ LUSC patients and non-VASH2+Snail+ patients, as well as between VASH2+VEGF-D+ patients and non-VASH2+VEGF-D+ patients.

A

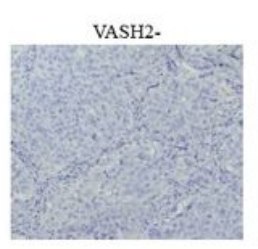

Snail-

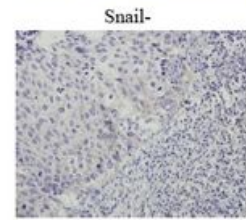

VEGF-D-

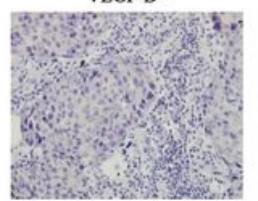

VASH2+

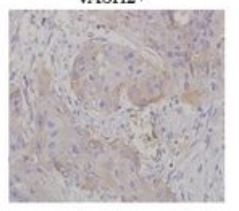

Snail +

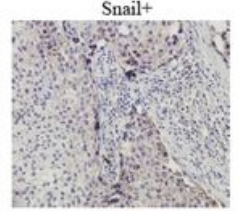

VEGF-D+

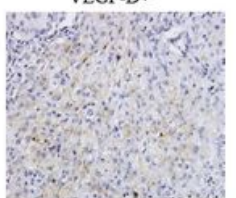

B
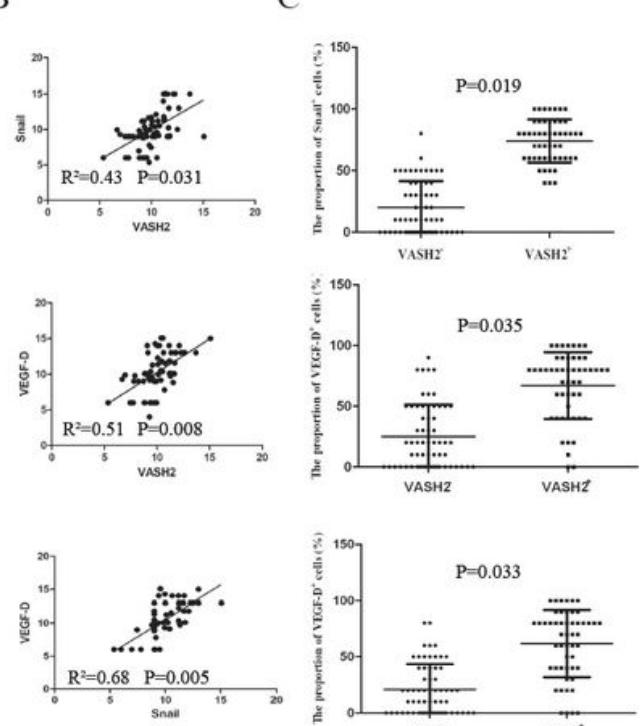

$\mathrm{D}$
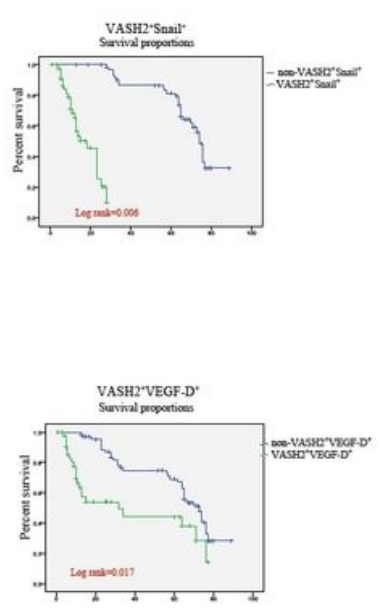
Figure 6

VASH2 was significantly correlated with either Snail orVEGF-D in primary LUSC tissues A The protein levels of Snail and VEGF-D in 64 cases of primary LUSC tissues were assessed using IHC staining assay. B We used qPCR to detect the mRNA levels of VASH2, Snail and VEGF-D in LUSC. C We analyzed the correlation of VASH2, Snail and VEGF-D by IHC results. D We divided them into double positive group. We compared the OS between VASH2+Snail+ LUSC patients and non-VASH2+Snail+ patients, as well as between VASH2+VEGF-D+ patients and non-VASH2+VEGF-D+ patients.

A
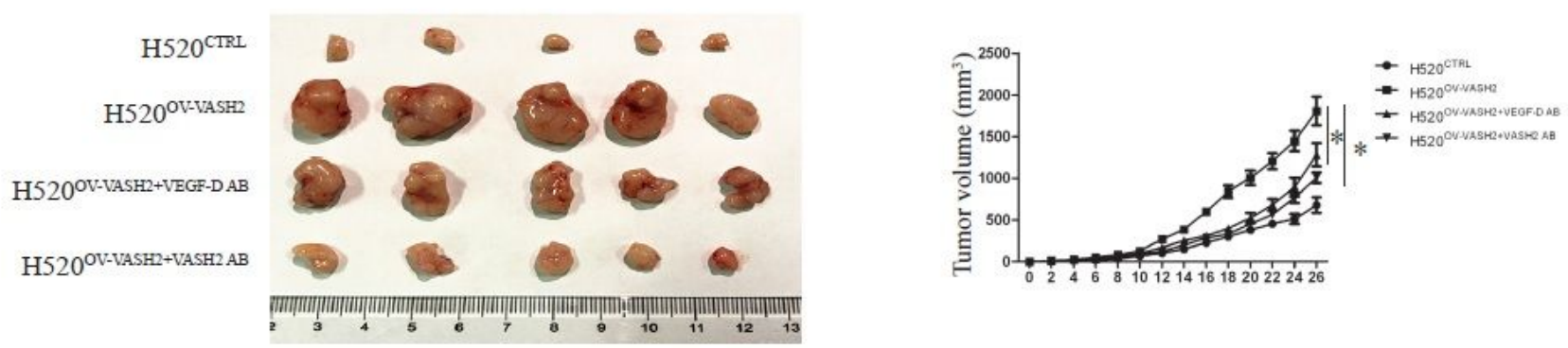

B

$\mathrm{H} 520^{\mathrm{CTRL}}$

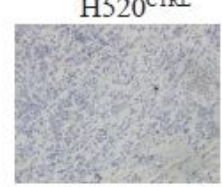

$\mathrm{C}$

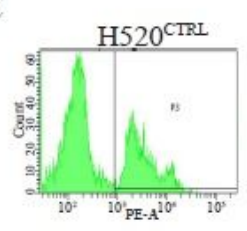

H520 ${ }^{\text {ov-VASH2 }}$
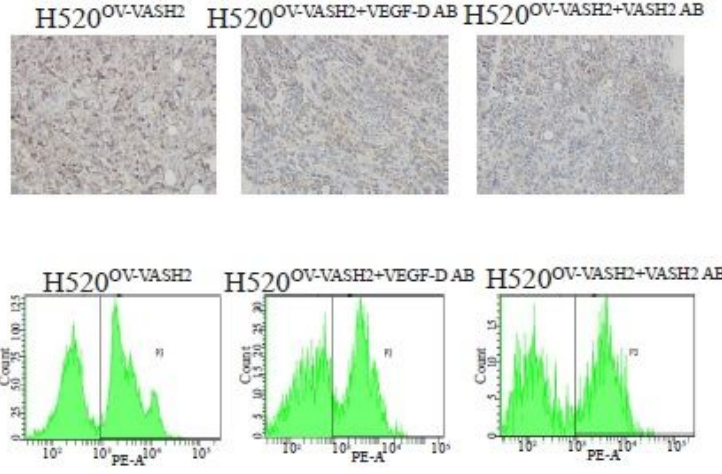

D
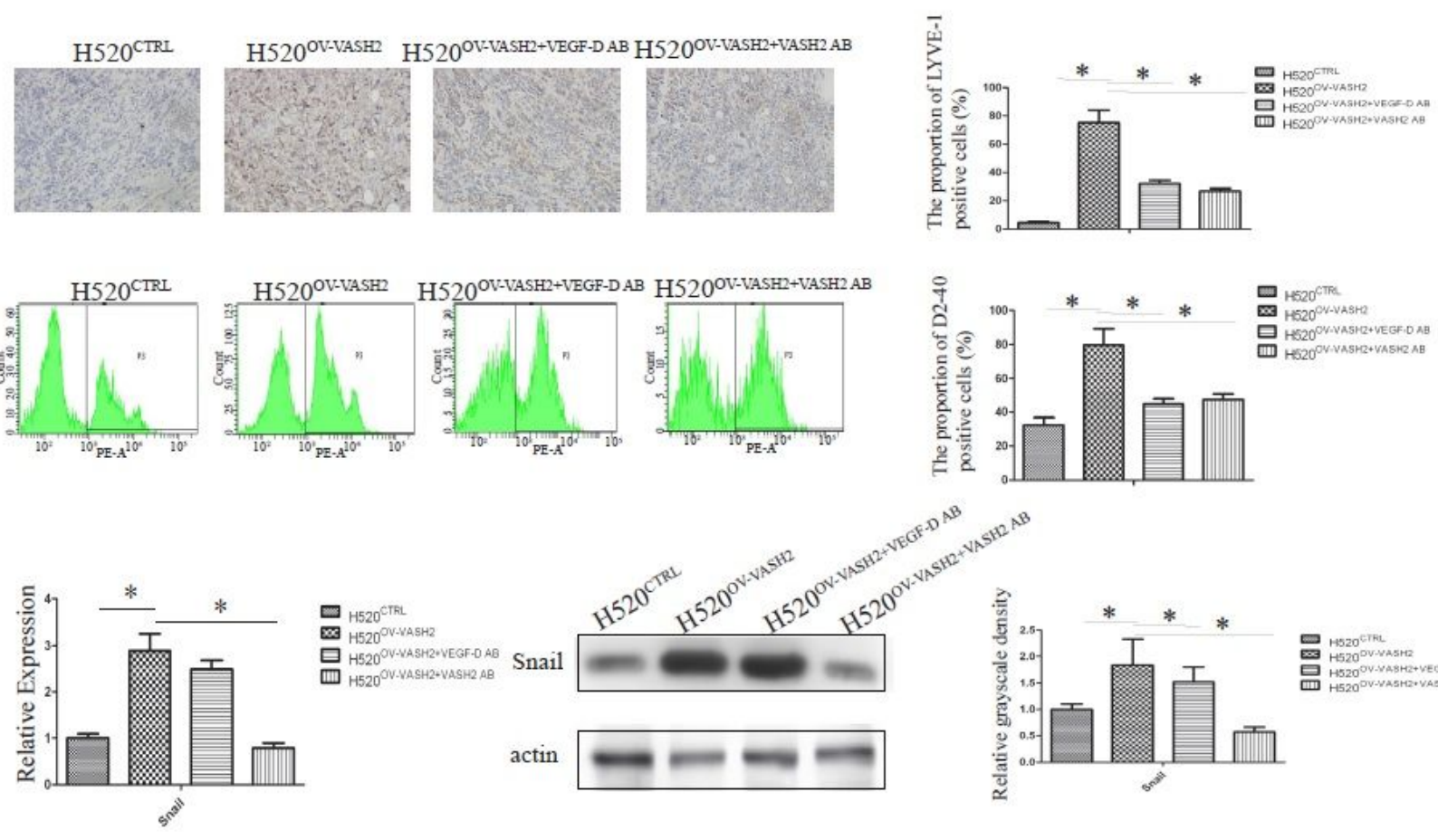

E

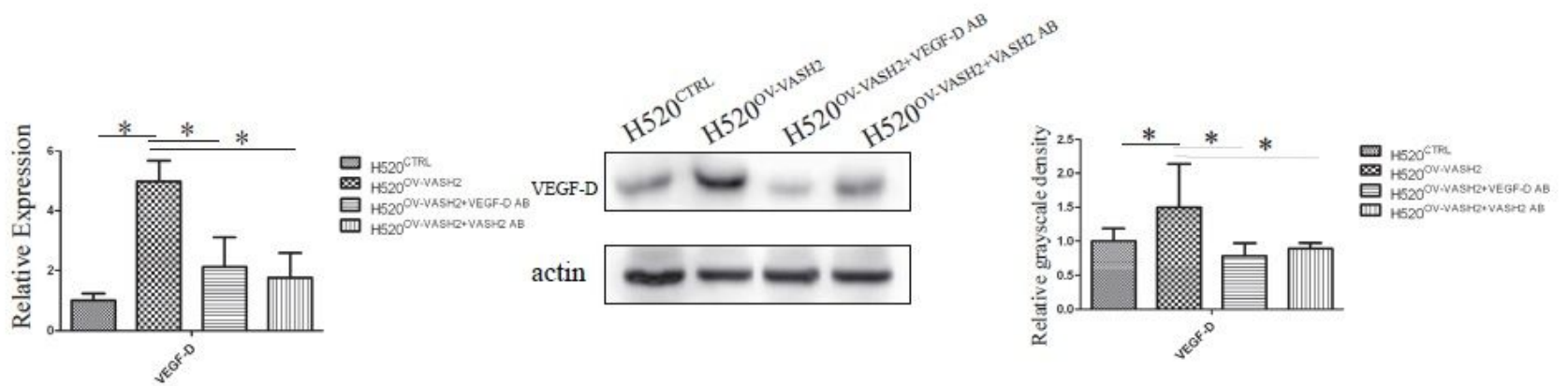

Figure 7 
VASH2 blocking antibody inhibited tumor growth and lymphangiogenesis by attenuating VEGF-D production A H520CTRL and H5200V-VASH2 were injected at 1×106 tumor cells/100 $\mu$ l per mouse subcutaneously into NOD-SCID mice. Once palpable tumors were formed (in 7-10 days) treatment cycles was initiated. The mice were anesthetized by intraperitoneal injection of $100 \mathrm{mg} / \mathrm{kg}$ anti-VEGFD and $10 \mathrm{mg} / \mathrm{kg}$ anti-VASH2. Treatment involved the mice being randomly divided into three groups, control, anti-VEGFD, and anti-VASH2 with each group containing 5 mice. The tumor sizes were monitored every 3 days. B Tumors were fixed and processed for IHC of the proportion of LYVE-1. C We used flow cytometry to detect the influence of VASH2 and VEGF-D on lymphoepithelial cells by labeling D2-40 positive cells. D, E We compared the mRNA and protein levels of Snail and VEGF-D between different groups using qPCR and WB assay. 
A
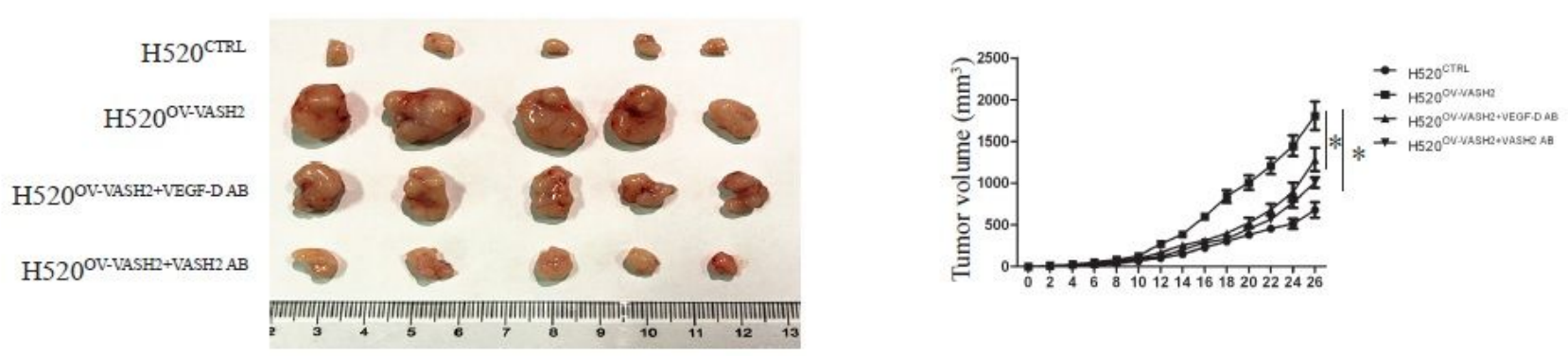

B
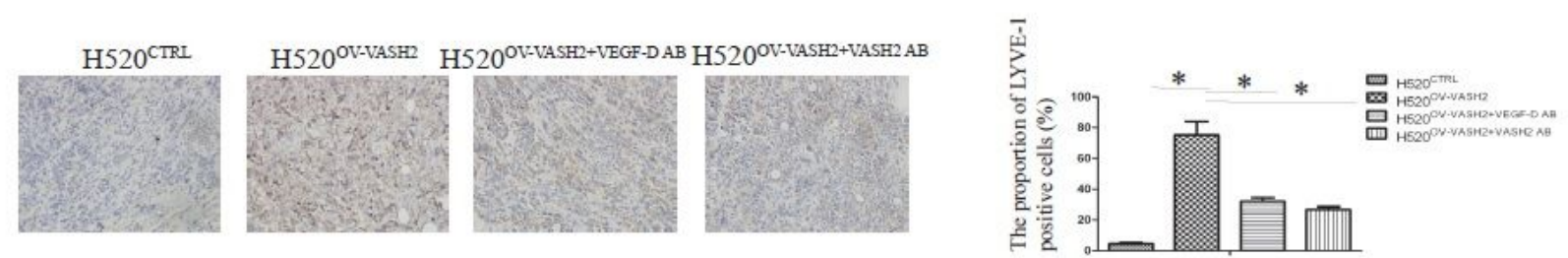

$\mathrm{C}$
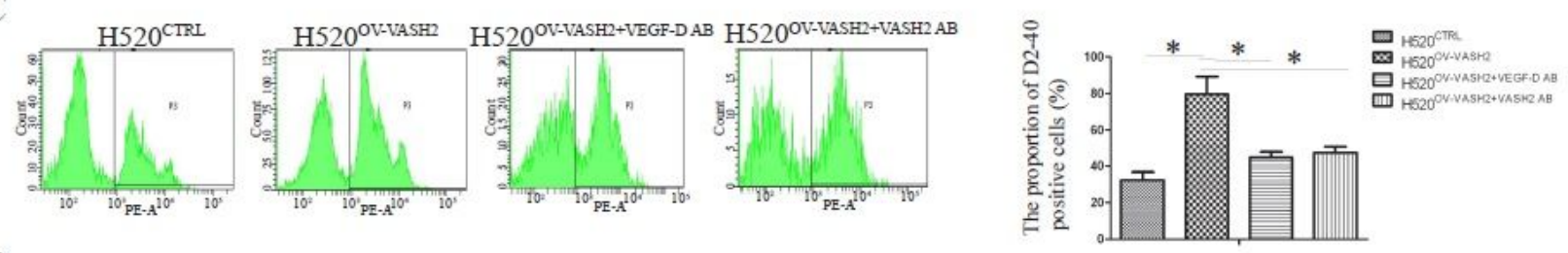

$\mathrm{D}$

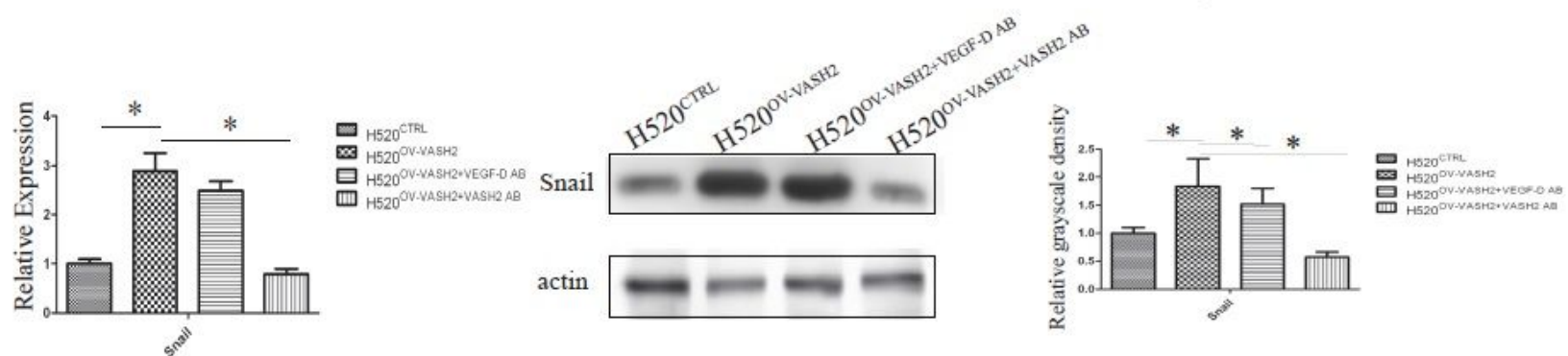

$\mathrm{E}$

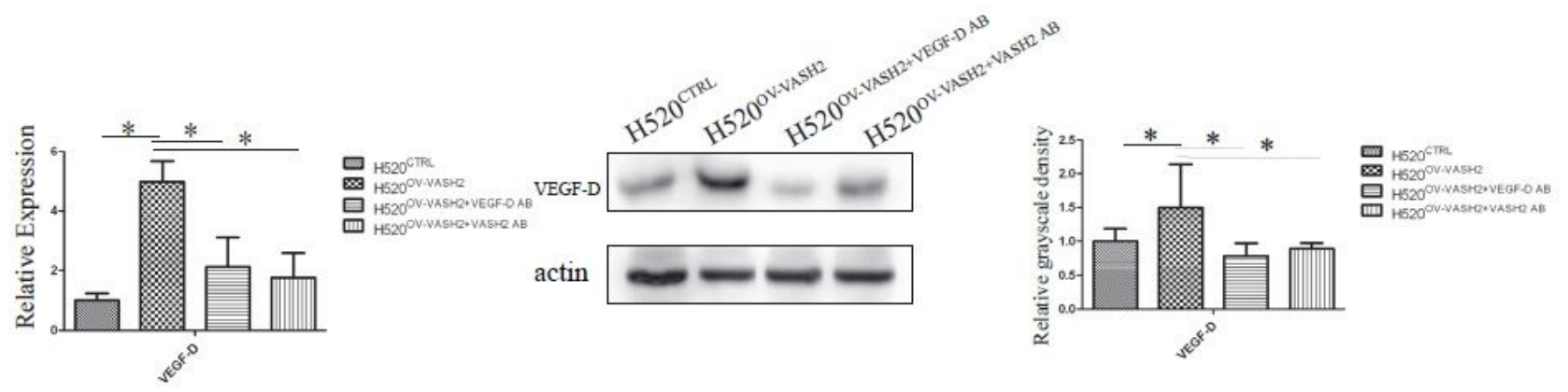

Figure 7

VASH2 blocking antibody inhibited tumor growth and lymphangiogenesis by attenuating VEGF-D production A H520CTRL and H5200V-VASH2 were injected at 1×106 tumor cells/100 $\mu$ l per mouse subcutaneously into NOD-SCID mice. Once palpable tumors were formed (in 7-10 days) treatment cycles was initiated. The mice were anesthetized by intraperitoneal injection of $100 \mathrm{mg} / \mathrm{kg}$ anti-VEGFD and $10 \mathrm{mg} / \mathrm{kg}$ anti-VASH2. Treatment involved the mice being randomly divided into three groups, control, anti-VEGFD, and anti-VASH2 with each group containing 5 mice. The tumor sizes were monitored every 3 
days. B Tumors were fixed and processed for IHC of the proportion of LYVE-1. C We used flow cytometry to detect the influence of VASH2 and VEGF-D on lymphoepithelial cells by labeling D2-40 positive cells. D, E We compared the mRNA and protein levels of Snail and VEGF-D between different groups using qPCR and WB assay.

A
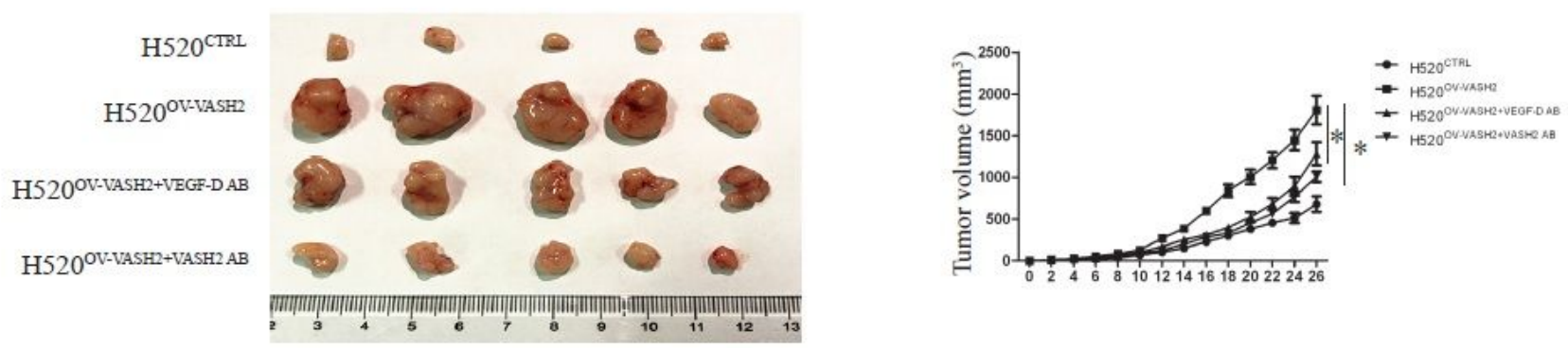

B
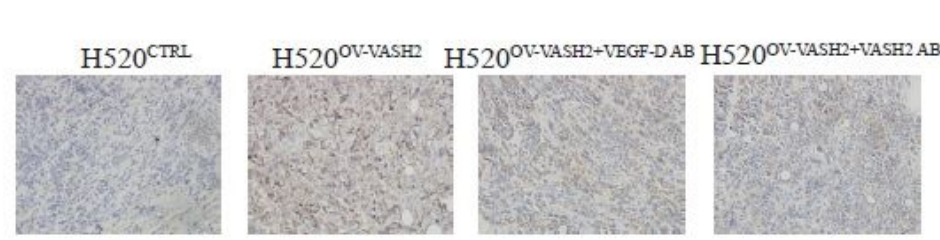

$\mathrm{C}$
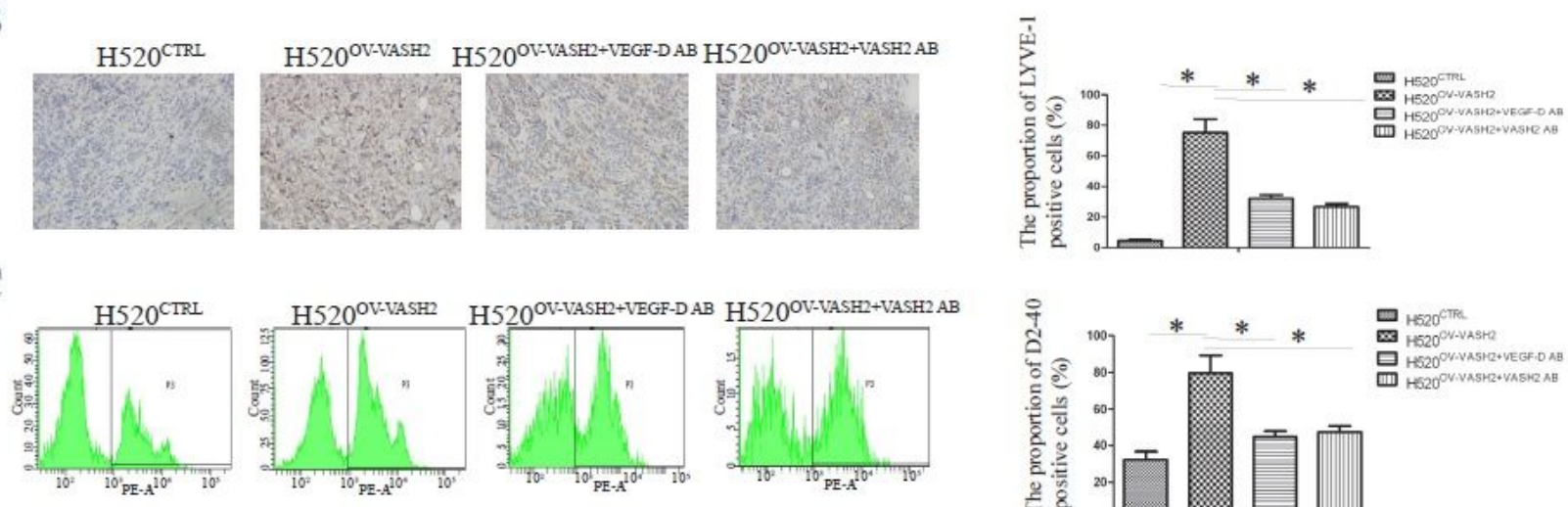

$\mathrm{D}$

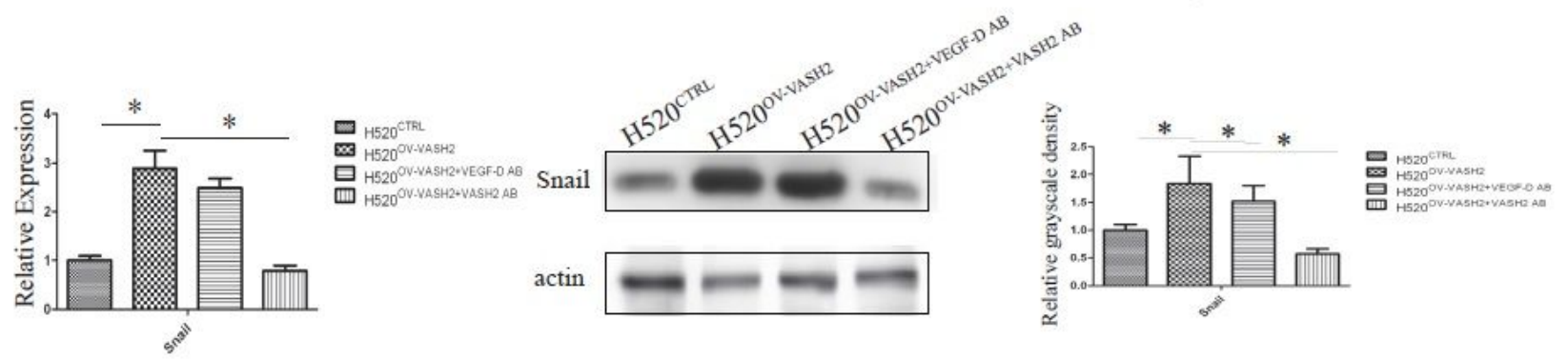

$\mathrm{E}$

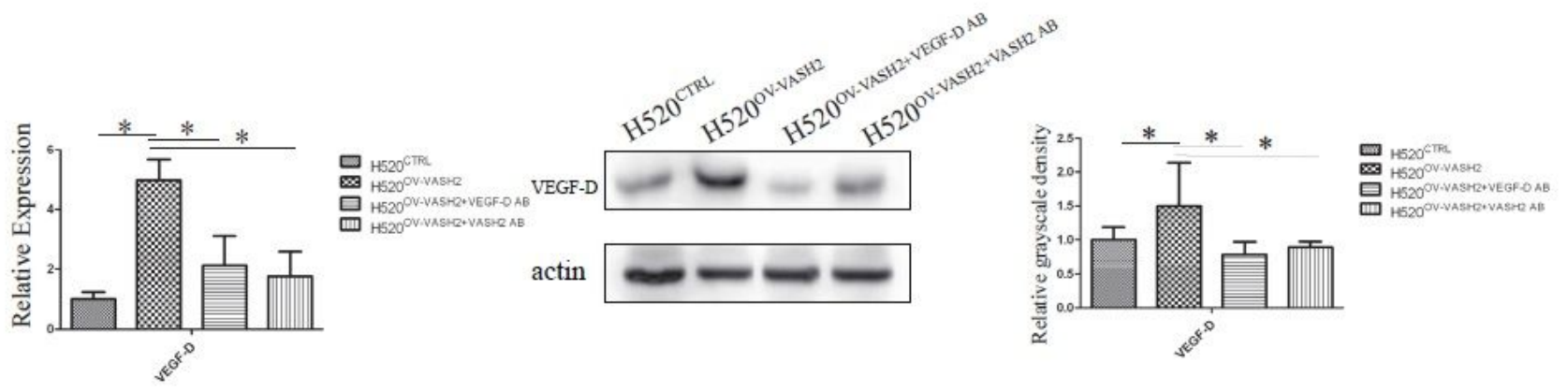

Figure 7

VASH2 blocking antibody inhibited tumor growth and lymphangiogenesis by attenuating VEGF-D production A H520CTRL and H5200V-VASH2 were injected at $1 \times 106$ tumor cells/100 $\mu$ per mouse 
subcutaneously into NOD-SCID mice. Once palpable tumors were formed (in 7-10 days) treatment cycles was initiated. The mice were anesthetized by intraperitoneal injection of $100 \mathrm{mg} / \mathrm{kg}$ anti-VEGFD and $10 \mathrm{mg} / \mathrm{kg}$ anti-VASH2. Treatment involved the mice being randomly divided into three groups, control, anti-VEGFD, and anti-VASH2 with each group containing 5 mice. The tumor sizes were monitored every 3 days. B Tumors were fixed and processed for IHC of the proportion of LYVE-1. C We used flow cytometry to detect the influence of VASH2 and VEGF-D on lymphoepithelial cells by labeling D2-40 positive cells. D, E We compared the mRNA and protein levels of Snail and VEGF-D between different groups using qPCR and WB assay.

A
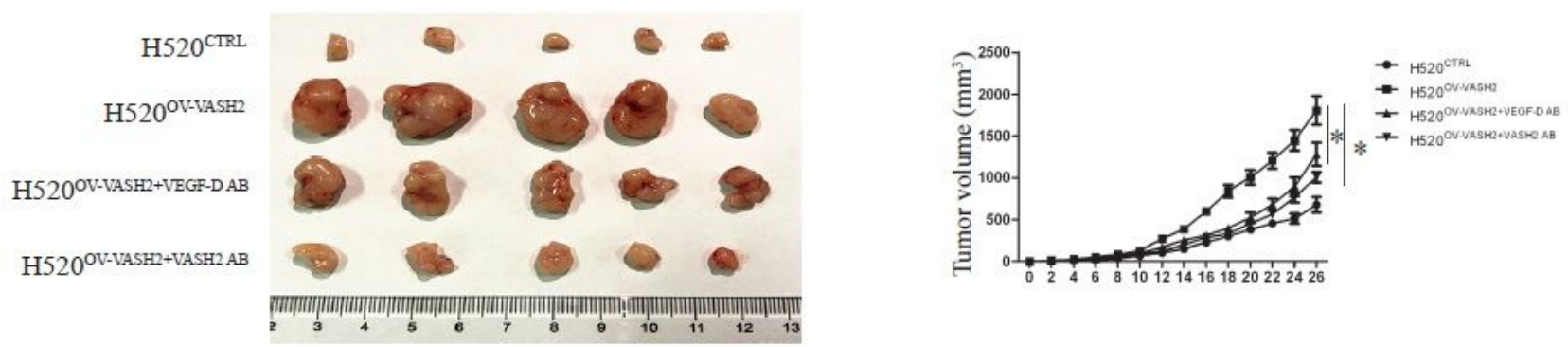

$\mathrm{B}$ $\mathrm{H} 520^{\mathrm{CTRL}}$
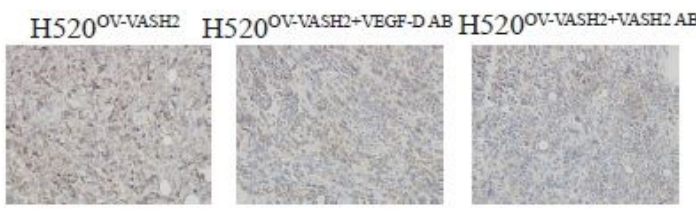

C

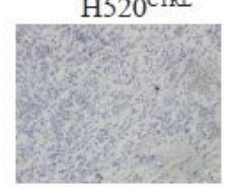

$\mathrm{H} 520^{\mathrm{CTRL}}$
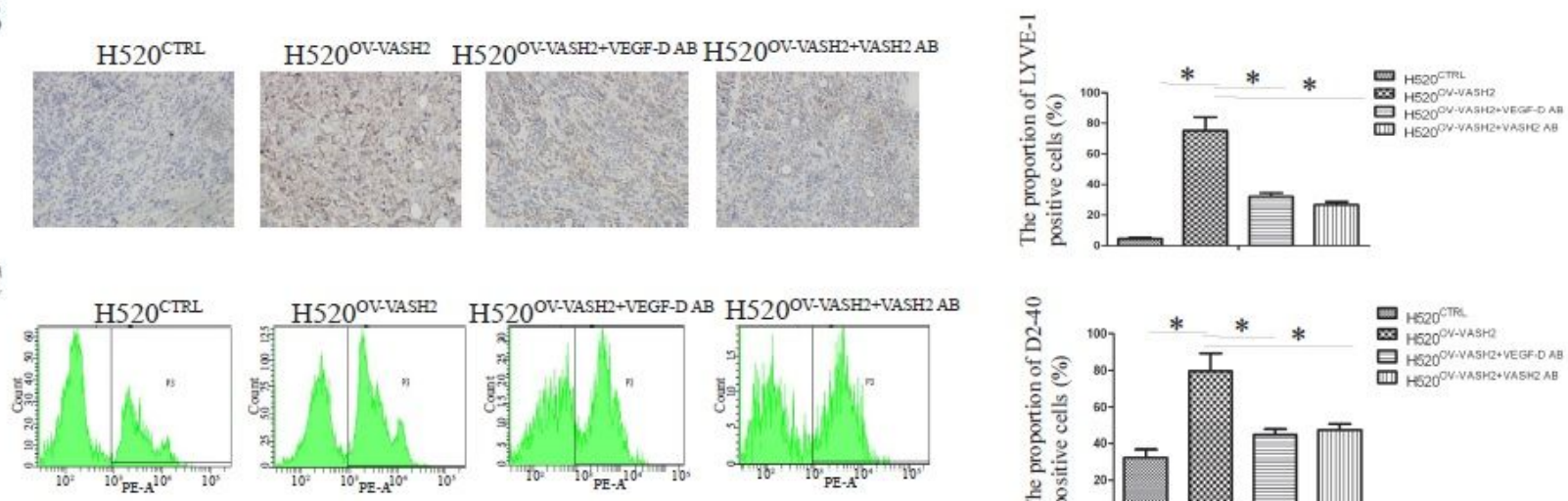

D

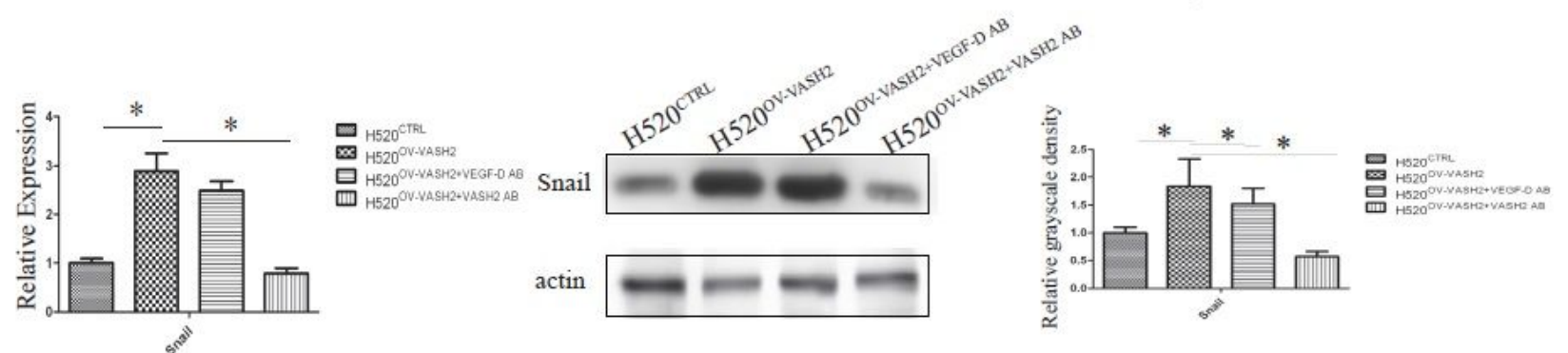

E

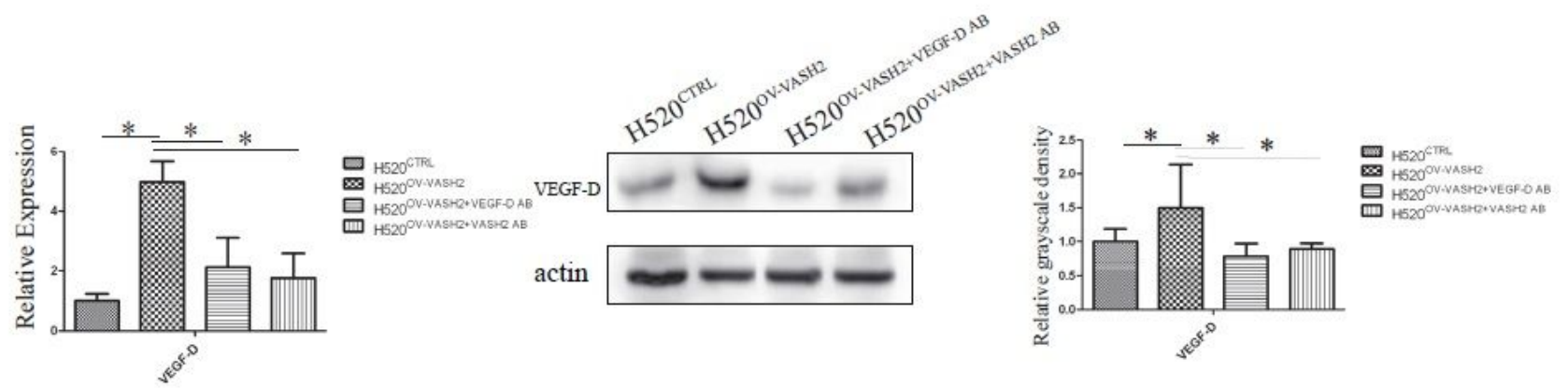




\section{Figure 7}

VASH2 blocking antibody inhibited tumor growth and lymphangiogenesis by attenuating VEGF-D production A H520CTRL and H5200V-VASH2 were injected at 1×106 tumor cells/100 $\mu$ l per mouse subcutaneously into NOD-SCID mice. Once palpable tumors were formed (in 7-10 days) treatment cycles was initiated. The mice were anesthetized by intraperitoneal injection of $100 \mathrm{mg} / \mathrm{kg}$ anti-VEGFD and $10 \mathrm{mg} / \mathrm{kg}$ anti-VASH2. Treatment involved the mice being randomly divided into three groups, control, anti-VEGFD, and anti-VASH2 with each group containing 5 mice. The tumor sizes were monitored every 3 days. B Tumors were fixed and processed for IHC of the proportion of LYVE-1. C We used flow cytometry to detect the influence of VASH2 and VEGF-D on lymphoepithelial cells by labeling D2-40 positive cells. D, E We compared the mRNA and protein levels of Snail and VEGF-D between different groups using qPCR and WB assay.

\section{Supplementary Files}

This is a list of supplementary files associated with this preprint. Click to download.

- S1.pdf

- S1.pdf

- S1.pdf

- S1.pdf

- S2.pdf

- S2.pdf

- S2.pdf

- S2.pdf

- Table1.pdf

- Table1.pdf

- Table1.pdf

- Table1.pdf

- Table2.pdf

- Table2.pdf

- Table2.pdf

- Table2.pdf 